\title{
Blind Equalization for Underwater Communications
}

Koen C.H. Blom 
Members of the dissertation committee:

Prof. dr. ir. G.J.M. Smit

Dr. ir. A.B.J. Kokkeler

Prof. dr. ir. C.H. Slump

Dr. ir. M.J. Bentum

Prof. dr. ir. S.M. Heemstra de Groot

Prof. dr. ir. A.-J. van der Veen

Dr. ir. H.S. Dol

Prof. dr. ir. P.G.M. Apers
University of Twente (promotor)

University of Twente (assistant-promotor)

University of Twente

University of Twente

Eindhoven University of Technology

Delft University of Technology

TNO

University of Twente (chairman and secretary)

\section{UNIVERSITY OF TWENTE.}

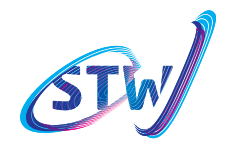

Enabling new technology

This research has been conducted within the STW SeaSTAR project (10552) and the STW RCPS-CD project (12699). This research is supported by the Dutch Technology Foundation STW, which is part of the Netherlands Organisation for Scientific Research (NWO) and partly funded by the Ministry of Economic Affairs.

CTIT Ph.D. Thesis Series No. 14-315

Centre for Telematics and Information Technology

University of Twente, P.O. Box 217, NL-7500 AE Enschede

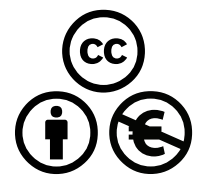

Copyright (C) 2014 by Koen C.H. Blom, Enschede, The Netherlands. This work is licensed under the Creative Commons AttributionNonCommercial 3.o Netherlands License. To view a copy of this license, visit http://creativecommons .org/licenses/by-nc/ $3.0 / \mathrm{nl} /$.

This thesis was typeset using $\mathrm{ETEX}_{2 \varepsilon}$, TikZ, and Vim. This thesis was printed by Gildeprint Drukkerijen, The Netherlands.

ISBN 978-90-365-3680-6

ISSN $\quad 1381-3617$ (CTIT Ph.D. Thesis Series No. 14-315)

DOI $10.3990 / 1.9789036536806$ 


\title{
BLIND EqUALIZATION FOR \\ Underwater COMMUNicATIONS
}

\section{ProefsChRIFT}

\author{
ter verkrijging van \\ de graad van doctor aan de Universiteit Twente, \\ op gezag van de rector magnificus, \\ prof. dr. H. Brinksma, \\ volgens besluit van het College voor Promoties \\ in het openbaar te verdedigen \\ op vrijdag 4 juli 2014 om 12.45 uur
}

door

Koen Cornelis Hubertus Blom

geboren op 7 december 1984

te Wanroij 
Dit proefschrift is goedgekeurd door:

Prof. dr. ir. G.J.M. Smit (promotor)

Dr. ir. A.B.J. Kokkeler (assistant-promotor)

Copyright (C) 2014 Koen C.H. Blom

ISBN 978-90-365-3680-6 


\section{Abstract}

Over $70 \%$ of Earth's surface is covered by water. Large parts of this immense water mass are still unexplored. Underwater wireless (sensor) networks would vastly improve man's ability to explore and exploit remote aquatic environments. Despite underwater sensor and vehicle technology being relatively mature, underwater communications is still a major challenge. As of today, due to the fast attenuation of light and radio waves in water, communication under water is mainly based on acoustic pressure waves to convey information. The most challenging characteristics of the underwater acoustic communication channel are its low and variable propagation speed, frequency-dependent attenuation and time-varying multipath propagation.

Spatial and spectral signal processing techniques can be employed to mitigate the effects of the distortion caused by the underwater acoustic channel. These signal processing techniques are usually implemented by means of filter operations. Their respective filter weights need to be adjusted continuously, since the underwater source, the scatterers, the medium and the receiver can be moving. In radio communication, training sequences are often used as a means to calculate appropriate filter weights. In general, the underwater channel capacity is scarce and underwater transmitters have limited energy resources. Therefore, to reduce energy consumption and to make more efficient use of the available capacity, this thesis elaborates on compensation of underwater channel distortion without employing training sequences. The latter is known as blind (adaptive) equalization.

To achieve a (relatively) high spectral and power efficiency, our underwater transmissions are assumed to be QPSK modulated. As a substitute for the missing training sequences, the constant modulus property of QPSK signals is exploited. Deviations of the equalizer output from a constant modulus act as a reference for weight updates. A well-known blind equalization method that uses this constant modulus property is the constant modulus algorithm (CMA).

No standard synthetic underwater acoustic channel model exists. Therefore, reallife experiments are performed for true testing. Current commercially available systems for underwater acoustic signal processing experiments make use of dedicated hardware. To implement other physical layer processing techniques (often) changes in hardware and/or proprietary firmware are required. This makes these systems unsuitable for our experiments. Therefore, a flexible multi-channel underwater testbed has been developed and used in experiments, to evaluate the performance of (novel) blind spatial equalizers. 
Two blind spatial equalization methods, which both exploit structural properties of the signal-of-interest, are presented in this thesis. The first method, the extended $C M A$ (E-CMA) is an algorithm known from the spectral equalization literature. In this thesis, the E-CMA is used in the context of spatial equalization where it is capable of updating the directionality of the array to improve signal reception, while simultaneously correcting for phase offsets. Initial results from our underwater experiments demonstrate the E-CMA's promising performance in the spatial equalization context. Compared to the conventional CMA, besides correcting phase offsets, the E-CMA exhibits faster convergence to the optimum mean square error level.

The second method for blind spatial equalization discussed in this thesis, is the angular CMA (A-CMA). In contrast to conventional adaptive methods, the A-CMA calculates steering angle updates instead of updates for the entire (filter) weight vector. This approach is attractive in array architectures where distribution of the steering angle is necessary, e.g., in mixed-signal hierarchical arrays. In these mixedsignal architectures, spatial equalization is performed on multiple levels, partly analog and partly digital. The desired steering angle can be calculated, by the ACMA, at the digital level of the hierarchy and thereafter be distributed to both the analog and digital spatial equalizers. The cost behaviour of the CMA and the ACMA is studied by simulation. Compared to the conventional CMA, the A-CMA provides faster convergence to a low mean square error level. Asymptotically, the mean square error (MSE) level of the CMA approaches the MSE level of the A-CMA.

In the multipath-rich underwater environment, reflections from current and previous transmissions add up constructively and destructively, causing frequencyselective distortion of the channel's magnitude and phase response. To compensate for frequency-selective channel distortion, blind spectral equalization is utilized. Since the propagation speed of underwater acoustic pressure waves is variable, a direct-path acoustic wave can arrive later than a reflected/refracted wave. This phenomenon, which is known as nonminimum-phase behaviour, complicates blind extraction of a channel's phase response.

Blind equalization of the magnitude and phase response of a nonminimum-phase channel can always be performed separately because a nonminimum-phase channel equalizer is decomposable into, respectively, a (i) minimum-phase and an (ii) allpass (nonminimum-phase) part. This thesis introduces a method for improving and accelerating blind equalization of a channel's all-pass response, known as the allpass CMA (AP-CMA). The all-pass CMA developed in this thesis can compensate a single nonminimum-phase zero. Compared to the CMA, it typically provides a faster and more accurate compensation of this zero.

Overall, based on simulated and empirical data, this thesis indicates that blind spectral and blind spatial equalization are appealing means for mitigation of distortion experienced in the underwater channel. 


\section{SAMENVATTING}

Meer dan $70 \%$ van het aardoppervlak is bedekt met water. Het overgrote deel van deze immense watermassa is nog onverkend. Omvangrijke draadloze onderwater (sensor) netwerken zullen hier verandering in brengen, ze stellen de mens in staat om afgelegen gebieden te verkennen en te exploiteren. Ondanks dat onderwater sensor- en voertuigtechnologie relatief volwassen zijn, staat onderwatercommunicatie nog in de kinderschoenen. Vanwege de sterke demping van licht en radiogolven geschiedt communicatie onder water meestal middels akoestische drukgolven, ofwel geluidsgolven. De meest uitdagende aspecten van akoestische communicatie onder water zijn de lage en variabele voortplantingssnelheid, de frequentie-afhankelijke demping en de tijdvariante multipad propagatie.

Spatiële en spectrale equalizatietechnieken kunnen worden toegepast om de verstoring van het onderwaterkanaal te corrigeren. Doorgaans worden deze vormen van signaalbewerking met filteroperaties geïmplementeerd. Gewichten van deze filteroperaties dienen voortdurend aangepast te worden wanneer de geluidsbron, de reflectoren, het medium en/of de ontvanger in beweging zijn. In radiocommunicatie is het gebruikelijk referentiesignalen te verzenden om filtergewichten te bepalen. In het algemeen is de capaciteit van het onderwaterkanaal beperkt en hebben onderwater geluidsbronnen een beperkte energievoorraad. Om zowel het energieverbruik van onderwatercommunicatie te reduceren als de beschikbare kanaalcapaciteit beter te benutten richt dit proefschrift zich op technieken om kanaalverstoring te compenseren zonder gebruik te maken van referentiesignalen. Deze vorm van equalizatie staat bekend als blinde adaptieve equalizatie.

Om energie-efficiënt een (relatief) hoge spectrale efficiëntie te behalen gebruiken we QPSK-modulatie voor onze onderwatertransmissies. In plaats van referentiesignalen wordt de inherente constante modulus eigenschap van QPSK-signalen benut voor adaptieve equalizatie. De afwijking van het ontvangen signaal van een constante modulus dient als referentie voor het aanpassen van de filtergewichten. Een welbekende blinde equalizatie methode die gebruik maakt van deze constante modulus eigenschap is het 'constant modulus algorithm (CMA)'.

Er bestaat geen standaardmodel voor het akoestische onderwaterkanaal. Praktijkexperimenten zijn noodzakelijk voor een realistische evaluatie van onderwater equalizatietechnieken. Huidige systemen voor onderwater akoestische signaalbewerking maken gebruik van gespecialiseerde hardware. Het aanpassen van de signaalbewerking op de fysieke laag van deze systemen vergt vaak aanpassingen in de hardware en/of gesloten broncode en maakt zulke systemen daarom ongeschikt voor onze 
experimenten. Om het functioneren van nieuwe blinde equalizatie algoritmes te evalueren is een flexibel meerkanaals onderwater signaalbewerkingssysteem ontworpen en in de praktijk gebruikt.

Twee blinde ruimtelijke equalizatiemethoden worden besproken in dit proefschrift. De eerste methode, het extended CMA (E-CMA), is een algoritme uit de spectrale equalizatie literatuur. In dit proefschrift wordt het gebruik van het E-CMA in een spatiële context onderzocht. Met behulp van experimenteel verzamelde datasets is aangetoond dat het E-CMA in staat is om richtingsveranderingen van het onderwater bronsignaal te compenseren en tegelijkertijd faseverschuivingen in het gebundelvormde signaal te corrigeren. Vergeleken met het CMA convergeert het E-CMA sneller naar de optimale gemiddelde kwadratische fout.

Het angular CMA (A-CMA) is de tweede methode voor blinde ruimtelijke equalizatie die in dit proefschrift beschreven wordt. In tegenstelling tot conventionele methoden past het A-CMA niet de filtergewichten aan, maar bepaalt het de stuurhoek. Deze aanpak is aantrekkelijk voor array architecturen waar distributie van de stuurhoek noodzakelijk is, zoals mixed-signal hiërarchische arrays. In deze mixedsignal arrays vindt ruimtelijke equalizatie op meerdere niveaus plaats; deels in de analoge en deels in de digitale hardware. De gewenste stuurhoek wordt door het A-CMA uitgerekend in het digitale deel van de hiërarchie en vervolgens gedistribueerd naar zowel de analoge als de digitale equalizers. De leercurve van het CMA en het A-CMA zijn bestudeerd middels simulatie. Vergeleken met het conventionele CMA convergeert het A-CMA sneller naar een lage gemiddelde kwadratische fout. Asymptotisch bereikt het CMA de gemiddelde kwadratische fout van het A-CMA.

Het akoestische onderwaterkanaal is een omgeving met doorgaans veel multipad propagatie. Interferentie van directe en gereflecteerde/gebogen transmissies veroorzaakt frequentieselectieve kanaalverstoring. Blinde spectrale equalizatie kan worden toegepast om deze frequentieselectiviteit te compenseren. Vanwege de variabele voortplantingssnelheid van akoestische drukgolven is het mogelijk dat een directe drukgolf pas later bij de ontvanger aankomt dan een gereflecteerde drukgolf. Dit fenomeen wordt niet-minimum-fase gedrag genoemd en het bemoeilijkt blinde extractie van het fase gedrag van het onderwaterkanaal.

Blinde equalizatie van de magnitude en fase respons van een niet-minimum-fase kanaal kan onafhankelijk worden uitgevoerd omdat een niet-minimum-fase equalizer altijd opgesplitst kan worden in een (i) minimum-fase en een (ii) allesdoorlaat (niet-minimum-fase) deel. Dit proefschrift introduceert een methode, genaamd het all-pass CMA (AP-CMA), om de blinde equalizatie van het niet-minimumfase deel te verbeteren. Het AP-CMA is geschikt voor compensatie van een enkele niet-minimum-fase nul. Vergeleken met het CMA is de compensatie van een nietminimum-fase nul door het AP-CMA sneller en nauwkeurig.

Concluderend, dit proefschrift toont aan dat blinde equalizatie een veelbelovende aanpak is om op een energie-efficiënte wijze voor onderwater akoestische kanaalverstoring te compenseren. 


\section{DANKWOORD}

Na vier jaar promoveren bij de vakgroep CAES zit het er nu echt op: mijn 'boekje' is afgerond. Mijn verleden bij CAES gaat echter langer terug dan deze vier jaar. Eind 2008, onder begeleiding van Gerard, André, Kenneth en Marcel, begon ik aan een afstudeeropdracht op het gebied van adaptieve bundelvorming. Na succesvolle afronding van mijn afstudeeropdracht bood Gerard mij een promotieplaats aan. Aanvankelijk was er twijfel, maar ik was (en blijf) van mening dat je altijd meer kunt leren en dit leek me een uitgelezen kans. Zodoende startte ik in 2010 met mijn promotieonderzoek op het gebied van digitale signaalbewerking voor onderwatercommunicatie. Verschillende mensen hebben bijgedragen aan de totstandkoming van het eindresultaat, en graag zou ik hen hieronder willen bedanken.

Er is één persoon waar ik de deur soms platgelopen heb, en dat is André. Hij wist, zelfs als mijn vraag verre van helder was, vaak al in welke richting ik naar antwoorden zou kunnen zoeken. Naast urenlange inhoudelijke discussies voor het whiteboard kwam er ook een breed scala aan andere onderwerpen ter sprake: van reisavonturen en verhuizingen tot aan wielrenroutes in Noordoost-Twente. André, ik wil je bedanken voor de fijne en waardevolle begeleiding. Ik heb het altijd plezierig gevonden om met je samen te werken.

De afgelopen vier jaar is CAES behoorlijk gegroeid. Gerard heeft het er als prof van deze groep meestal flink druk mee. Desondanks staat zijn deur altijd open en worden ingeleverde stukken razendsnel van nuttige kritiek voorzien. Gerard, ik ben je dankbaar voor de vele discussies en het snelle commentaar, maar vooral dat je mij deze interessante kans hebt geboden.

Tijdens mijn promotietraject heb ik verschillende afstudeerders begeleid: Fasil, Marco, Hubert en Jordy. Het overleg met afstudeerders was een welkome afleiding en bood vaak nieuwe inzichten. Heren, bedankt dat jullie zo verstandig zijn geweest om zulke uitdagende afstudeeropdrachten te kiezen.

De laatste maanden zijn behoorlijk druk geweest. Gelukkig hebben we secretaresses die het hoofd koel houden in hectische tijden: Marlous, Thelma en Nicole. Naast de serieuze zaken vond ik het altijd gezellig om bij jullie een praatje te maken. Andere bronnen van gezelligheid zijn de pauzes en borrels. Sterke verhalen uit bijna-Zeeland tot diep in Friesland passeerden de revue. Er zijn weinig andere vakgroepen waar meer Fries dan Engels wordt gesproken. Sinds het vertrek van Maurice heeft het mij behoorlijk wat inspanning gekost om het Brabantse vaandel hoog te houden. Beste collega's, hartstikke bedankt voor de serieuze en vooral ook de minder serieuze gesprekken. 
Een aantal personen zijn direct betrokken geweest bij mijn promotietraject en de afronding van dit proefschrift. Graag wil ik hen kort even bedanken. Kamergenoot Christiaan voor de gezelligheid en interessant overleg; deeltijdkamergenoten Robert, Arjan, Jochem en Marco voor de aangename sfeer in hun wedkantoor; Jochem voor het LTEX-template en het redigeren van tekst; Wim en Marco voor energieke discussies en feedback op het proefschrift; Hermen voor zijn droge humor en zijn spelfoutfetish; Kenneth, Niels, Mark, Wouter en Nirvana voor hun hulp tijdens het dive-center experiment; Jan voor het vinden van een passend vervolgproject (en de bierproef); Mark voor de inhoudelijke discussies (voor of na de koffie++); Tuncay and other people from SUASIS Underwater Systems for their help during experiments; Saifullah to help me understand transducer drivers; Bart voor het lezen van een aantal hoofdstukken; Henry voor de discussies over onderwaterkanaalmodellering en de hele promotiecommissie voor hun feedback op het proefschrift.

Zoals bij veel promovendi heeft mijn sociale leven aardig wat klappen opgelopen. Gelukkig waren er personen die de schade binnen de perken wisten te houden en ik wil enkelen daarvoor in het bijzonder bedanken. Bart en Willem voor de altijd gezellige kroeg- en stapavonden in jullie stadsjie; Henze voor de wielrentochten, series en biertjes; Kenneth en Marlies voor de leuke gesprekken in het boemeltje en de biertjes/wijntjes in Arnhem, Zutphen en Enschede; Joekskapel Sodejuu voor de vele avonden Brabantse gezelligheid; pandgenoten voor een kop koffie of een biertje; Martien en Inge voor het starten van een reeks toffe \#ASN-feestjes; Elwin, Gerard, Chris en Marco voor de verjaardagsfeestjes en Bram voor de gezelligheid tijdens schrijfmarathons op de vijfde vloer.

Er zijn twee vrienden die zowel persoonlijk als inhoudelijk hebben bijgedragen aan mijn promotie: Marco en Rinse. Marco, gedurende hardlooptrainingen, barbecues, verhuizingen, feestjes en andere gelegenheden zijn talloze onderwerpen aan bod gekomen. Digitale signaalbewerking en bundelvorming maakten daar een belangrijk deel van uit. Ooit zou de dag komen dat mijn promotie ten einde ging lopen; fijn dat je mij op die dag als paranimf bij wilt staan. Rinse, gedurende mijn promotie heb ik jou steeds beter leren kennen. Of het nou tot middernacht solderen, carnaval in Oeteldonk, of knallen op een zeilboot is, jij vindt het allemaal prachtig; ik vind het prachtig dat jij mij als paranimf bijstaat.

Tenslotte wil ik mijn ouders bedanken voor de steun. Wellicht kan dit proefschrift jullie een beetje meer inzicht geven in mijn werkzaamheden van de afgelopen jaren.

Koen,

Rijkevoort-De Walsert, juni 2014 


\section{Contents}

1 INTRODUCTION 1

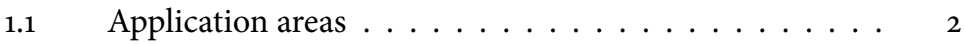

1.2 Underwater-acoustic sensor networks ........ 4

1.2.1 Sensor node deployment ........... 4

1.2.2 Network topology ........... . 4

1.3 Water - a challenging communication medium .... 5

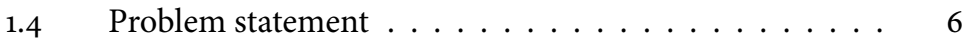

1.5 Approach ..................... 7

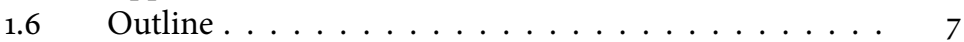

1.7 Notational conventions ............. 7

2 THE UNDERWATER CHANNEL 9

2.1 Introduction . . . . . . . . . . . . 9

2.2 Terminology and units ............... 10

2.2.1 Acoustic pressure waves .......... 10

2.2.2 Acoustic intensity ............ 10

2.3 Transmission loss and ambient noise ......... 11

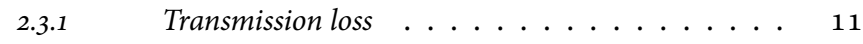

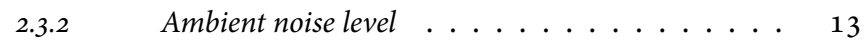

2.4 Variable propagation speed ............. 15

2.4.1 Sound speed profile ............ 16

2.4.2 Shadowing ............... 17

2.4.3 Acoustic waveguide ............. 19

2.5 Multipath propagation .............. 19

2.5.1 Deterministic time-invariant multipath model . . 20

2.5.2 Deterministic time-varying multipath model . . 20

2.5.3 Stochastic time-varying multipath model .... 21

2.5.4 Nonminimum-phase behaviour ........ 22

2.6 Conclusions ................. 23

3 MULTI-CHANNEL UNDERWATER TESTBED 25

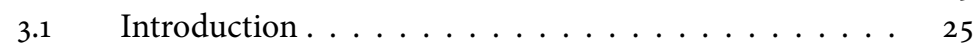

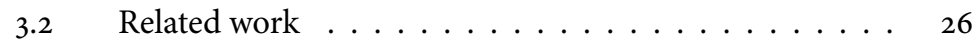

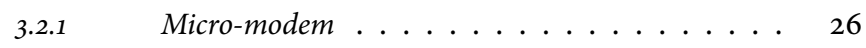

3.2.2 Reconfigurable modem .......... 27

3.3 Requirements ................ 27 
3.4 System-level design . . . . . . . . . . . . . 28

3.4.1 Choice of operating bandwidth and transducer . . 28

3.4.2 Array configuration ....................... 30

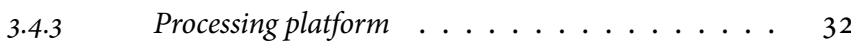

3.5 System implementation . . . . . . . . . . . 32

3.5.1 Memory-mapped system-on-chip architecture . . . 34

3.5.2 Streaming system-on-chip architecture . . . . . 35

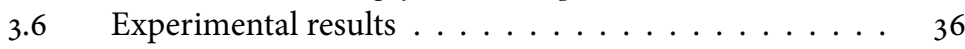

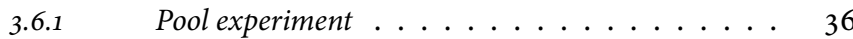

3.6.2 Dive-center experiment . . . . . . . 37

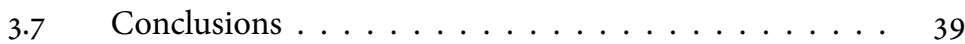

4 SPATIAL EQUALIZATION 41

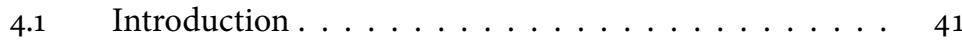

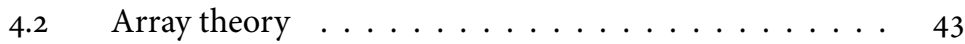

4.2.1 Array topologies .............. 43

4.2.2 Near- and far-field condition . . . . . . . . 44

4.2.3 Far-field source position . . . . . . . . . 45

4.2.4 Time delay and array manifold vector . . . . . . . . 46

4.2.5 Array processing ... . . . . . . . . 47

4.2.6 Array response vector and beamformer response . . 49

4.2.7 Performance analysis . . . . . . . . 51

4.3 Adaptive array processing . . . . . . . . . . . 54

4.4 The constant modulus algorithm . . . . . . . . . . 56

$4.4 .1 \quad$ History . . . . . . . . . . . . 56

$4.4 .2 \quad$ CMA cost function . . . . . . . . . . . . 56

4.4.3 CMA cost minimizer . . . . . . . . . . . 57

4.4.4 Computational complexity ............... 58

4.4.5 Empirical performance ........... 59

4.5 The extended constant modulus algorithm . . . . . . 62

4.5.1 E-CMA cost function . . . . . . . . 62

4.5.2 E-CMA cost minimizer . . . . . . . 63

$4.5 .3 \quad$ Computational complexity . . . . . . . 64

4.5.4 Empirical performance. . . . . . . . . 64

4.6 The angular constant modulus algorithm . . . . . . 65

4.6.1 A-CMA cost criterion and minimizer . . . . . 66

4.6.2 Error-performance surface . . . . . . . . . 69

4.6.3 Complexity Analysis . . . . . . . . . . 70

4.6.4 Simulation Results ................ 70

4.7 Conclusions and future work . . . . . . . . 72

5 SPECTRAL EQUALIZATION 75

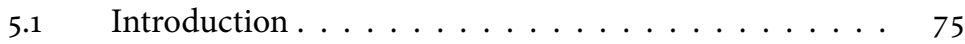

5.2 Theoretical background . . . . . . . . . 78

5.2.1 Blind adaptive equalization ........ 78 
5.2.2 Minimum-phase/all-pass decomposition . . . 78

5.2.3 Nonminimum-channel equalization . . . . . . 79

5.3 Dimensionality reduction of the CMA . . . . . . . 79

5.3.1 All-pass channel and equalizer........ 80

5.3.2 Single-pole single-zero All-Pass CMA . . . . . 81

5.3.3 Error-performance Surface......... 82

5.4 The AP-CMA cost minimizer ........... 83

5.4.1 Wirtinger Calculus ................. 83

5.4.2 Minimizer Derivation ........... 84

5.5 Simulations ................. 86

5.5.1 Convergence Behaviour Analysis . . . . . . . 86

5.5.2 Equalization Performance Comparison ..... . 87

5.6 Conclusions and future work ........... 88

6 CONCLUSIONS AND FUTURE WORK 91

6.1 Blind equalization for underwater communications . . . 91

6.1.1 Contributions .............. 92

6.1.2 Recommendations ........... 92

A IDENTIFIABILITY OF NONMINIMUM-PHASE CHANNELS 95

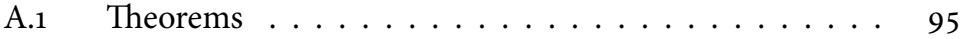

A.1.1 Benveniste-Goursat-Ruget ......... 95

A.1.2 Shalvi-Weinstein ........... 95

$\begin{array}{ll}\text { ACRONYMS } & 99\end{array}$

$\begin{array}{ll}\text { NOMENClatURE } & 103\end{array}$

$\begin{array}{lr}\text { BIBLIOGRAPHY } & 107\end{array}$

$\begin{array}{ll}\text { List of Publications } & 115\end{array}$ 



\section{1 \\ INTRODUCTION}

The first known record of underwater acoustics dates back to the time of the Greek philosophers. In the fourth century BC, Aristotle noted that sound can be heard in water as well as in air [7]. A long time after Aristotle, in the fourteenth century, Leonardo da Vinci discovered a practical application of underwater acoustics. In his notebook he wrote: "If you cause your ship to stop and place the head of a long tube in the water and place the outer extremity to your ear, you will hear ships at a great distance from you." Experimental research on underwater acoustics got really off the ground during the last two centuries. An experiment worth mentioning is the determination of the underwater acoustic propagation speed by Colladon and Sturm in 1826 [42]. In a boat on Lake Geneva, Sturm struck a submerged bell and simultaneously generated a flash of light. The flash signalled Colladon, at a large distance of the bell, to start a watch until the underwater sound was heard. Their measured propagation speed of $1435 \mathrm{~m} \mathrm{~s}^{-1}$ is close to the modern value of $1438 \mathrm{~m} \mathrm{~s}^{-1}$ (for freshwater with a temperature of $8^{\circ} \mathrm{C}$ ). Later, at the end of the nineteenth century, the striking of submerged bells on lightships became an important tool for ship navigation.

In the twentieth century, the inefficient pneumatically and electrically operated submerged bells got replaced by other types of acoustic sources. The first practical underwater transducer was designed by Fessenden in 1913. A year later this 'Fessenden oscillator' was used for echolocation of an iceberg at $3.2 \mathrm{~km}$ distance [7]. The need for localizing icebergs became tragically clear after the tragedy with the Titanic in April 1912.

In the years that followed, at the onset of World War I, the main focus of underwater acoustics became detection of submarines in both shallow- and deep-waters. Initially, French researchers focused on echolocation techniques with active transmitters and the British on passive listening. These methods are now respectively known as active and passive sound navigation and ranging (SONAR). Considerable progress has been made when the French and the British started sharing their results. The first active SONAR that obtained echos from a submarine, at almost $500 \mathrm{~m}$ distance, was built in England by Boyle in 1918 [1].

The period between the two World Wars led to more advances in seismic surveying and echolocation. Applications were, e.g., sea floor mapping and detecting fish 
shoals [7]. In the same era, the understanding of underwater acoustic propagation grew significantly. An important discovery was that of the acoustic shadow zone, an area where submarines could not be detected with acoustic echolocation methods.

During World War II, underwater acoustics received high priority in Europe, the USA and the Far East. Improved transducers, better understanding of acoustic propagation and advances in electronics resulted in practical systems [72]. After the war, the US National Defense Research Committee wrote an extensive collection of reports concerning underwater acoustics based on wartime achievements [57].

Based on wartime studies, American and Russian scientists discovered that at certain depths, the ocean acts as a waveguide for low-frequency acoustic signals. In 1946, this worldwide permanent sound channel was termed the sound fixing and ranging (SOFAR) channel [19]. The SOFAR channel has many interesting applications, e.g., (i) tracking ocean currents using sound-emitting neutrally buoyant floats, (ii) localizing submarine earthquakes and (iii) localizing submerged submarines [56]. Neutrally buoyant floats are objects with an equal tendency to float as to sink and can therefore maintain a particular depth in the ocean.

Mathematically, underwater sound propagation can be modelled using the wave equation. An important set of solutions to the wave equation are the normal modes. In 1948, Worzel and Ewing et al. used the normal mode theory to predict long-range propagation in shallow water [92]. In subsequent underwater experiments their theory became remarkably useful to interpret gathered data [60].

During the Cold War, research emphasis shifted to deep water [37]. The US navy positioned a large network of acoustic arrays in the SOFAR channel to keep track of Soviet submarines. Measurements of these arrays were transmitted to coastal stations for further analysis, via undersea telephone cables. This multi-billion dollar underwater network was termed sound ocean surveillance system (SOSUS) and can be regarded as an important technical achievement during the Cold War.

The development of the transistor in the late forties led to an exponential increase of available computing power during the second half of the twentieth century. Models of underwater acoustic propagation became much more sophisticated and the available processing power of underwater equipment increased dramatically. Being able to execute digital algorithms opened a new era for underwater acoustic communication: from a fairly primitive underwater telephone, developed in the mid-forties for communication with submarines, to systems that can achieve tens of kbps data throughput [77].

\subsection{Application AREAS}

Historically, underwater acoustic research almost exclusively focused on military applications. Improvement of transducer technology, advances in analog electronics and the wide availability of digital processing power led to a whole new range of both commercial and military applications. One such application, which offers 
a lot of potential, is underwater wireless monitoring. This subject is investigated within the SeaSTAR project, partly funded by STW under the ASSYS program. The objective of the SeaSTAR project is to investigate, define and develop core technologies for underwater wireless monitoring. Broadly speaking, underwater monitoring applications can be categorized into (i) oil and gas exploitation, (ii) environmental monitoring, (iii) safety and security monitoring.

In the category of oil and gas exploitation, pipeline monitoring is an important application. Underwater pipelines can be very long; the longest underwater pipeline today stretches a length of $1200 \mathrm{~km} \mathrm{[13].} \mathrm{Sensors} \mathrm{performing} \mathrm{measurements} \mathrm{of}$ pressure, corrosion, vibration and acoustic phenomena can be used to distinguish sections of pipeline susceptible to leakage. Acoustic modems attached to these sensors provide a means to transmit vital monitoring data to surveillance operators.

Environmental monitoring can be categorized in (i) water quality and acoustic pollution monitoring, (ii) ocean current monitoring and (iii) biological monitoring [93]. Construction noise and operating noise from offshore wind farms are well-known examples of acoustic pollution. Acoustic pollution monitoring during construction of the Princess Amalia offshore wind farm is illustrated in figure 1.1. The second category of environmental monitoring, observation of ocean currents, is a necessity to improve weather forecasts and to better understand climate fluctuations. The focus of biological monitoring is to gain more knowledge of marine ecosystems. In the Netherlands, the Royal Netherlands Institute for Sea Research facilitates and supports this type of applied marine research.

Safety and security monitoring encompasses, for example, ship and submarine detection in harbors. In addition to detecting underwater vehicles, passive diver detection in harbors also gained a lot of interest recently [80]. Divers are able to place small drug smuggling containers on the hull of vessels or pose a substantial threat when carrying explosive devices. A typical example of safety monitoring is a real-time tsunami warning system. Such a system could transmit acoustic tsunami warnings based on seismic monitoring of the ocean floor.

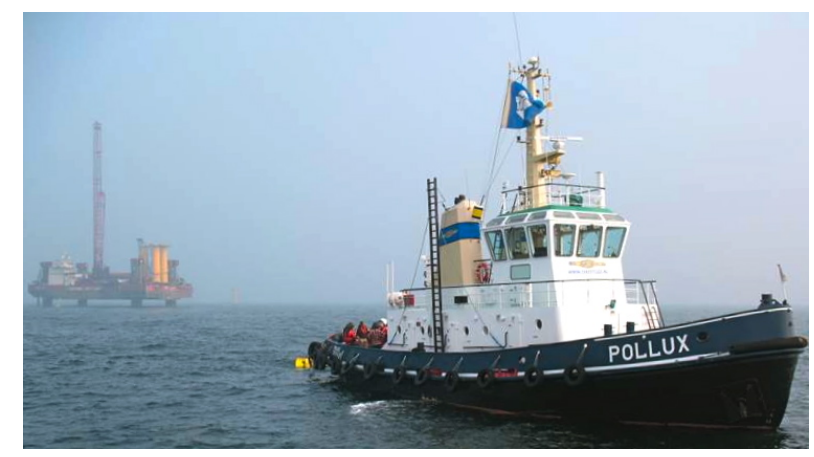

FIGURE 1.1 - Monitoring underwater noise levels during pile driving [53]. 
In the SeaSTAR project, underwater-acoustic sensor networks (UW-ASNs) are considered a core technology to realize wireless monitoring of the aquatic environment. Historically, underwater wireless monitoring mainly relied on point-to-point communication between a sensor node and a gateway node on a fixed location. In contrast, UW-ASNs are composed of multiple acoustic sensor nodes that collaboratively perform monitoring in a certain region of interest. Acoustic sensor nodes consist of energy storage and power control, sensing, data processing and acoustic communication hardware integrated in a watertight housing. A key feature of UW-ASNs is the cooperative effort of the nodes. Instead of transmitting raw data to every other node, sensor nodes exchange data with nearby nodes, perform local processing and transmit only the required (and partially processed) data.

In general, the system architecture of a UW-ASN is related to the following aspects: (i) the topology of the network, (ii) equipment in terms of hard- and software, (iii) connectivity and (iv) communication protocols. Methods for sensor node deployment and different network topologies are discussed in the upcoming sections. The other aspects are discussed in subsequent chapters.

\subsubsection{SENSOR NODE DEPLOYMENT}

Underwater sensor nodes can be deployed randomly or accurately positioned by, e.g., divers. Given a certain deployment technique, the minimum number of sensor nodes to meet the required sensing and communication coverage can be calculated. For random and triangular deployments, this is covered by Pompili et al. [61]. In their work, the trajectory of sinking objects is evaluated to compute the deployment surface area given the targetted ocean bottom area.

\subsubsection{NETWORK TOPOLOGY}

The topology of a UW-ASN refers to the arrangement of the sensor nodes in space and is crucial for the energy consumption, capacity and reliability of the network [2]. Of utmost importance is the question whether the UW-ASN primarily has an oceanbottom or an ocean-column topology.

ocean-bottom topology

A UW-ASN with an ocean-bottom topology primarily encounters acoustic links with sound pressure waves propagating in parallel to the ocean bottom. A horizontal acoustic channel is (strongly) affected by time-varying multipath propagation, caused by reflections of the sea surface and ocean bottom [2]. 
ocean-column topology

A UW-ASN with an ocean-column topology primarily encounters acoustic links with sound pressure waves propagating perpendicular to the ocean bottom. The vertical acoustic channel can experience a small amount of multipath propagation. Compared to the ocean-bottom topology, its time-variance is less severe [2].

An example of a network architecture with primarily an ocean-bottom topology is a pipeline monitoring architecture. For pipeline monitoring, nodes are fixed to a pipeline and use horizontal communication for data transmission to neighbouring nodes. An example of an architecture with an ocean-column topology is a system that continuously monitors the underwater propagation speed at various depths.

Transmission of vital data over a medium- to long-range distance can be accomplished through a collaborative effort of multiple nodes. For instance, a cluster of distinct nodes can act as a (phased) array and hence electronically compensate for changing channel conditions and source directions.

\subsection{WATER - A CHALLENGING COMMUNICATION MEDIUM}

As of today, due to the fast attenuation of light and electromagnetic (EM) waves in water, underwater communication is mainly based on acoustic pressure waves [86]. The underwater acoustic environment is a harsh environment for communication; its unique properties pose significant challenges for the design of UW-ASNs. The three main challenges of underwater acoustic communication are the (i) low propagation speed, (ii) frequency-dependent attenuation and (iii) time-varying multipath propagation [79]. A short introduction to these characteristics is given here. In chapter 2, we will give a more thorough and quantitative analysis of the underwater channel.

The propagation speed of underwater acoustic pressure waves is variable and determined by salinity, temperature and depth of the water. In underwater communication, a direct-path acoustic pressure wave can arrive later than a reflected wave due to the variation in propagation speed while travelling through the medium. This phenomenon is called nonminimum-phase behavior and it makes restoration of the received signal more complicated.

As the acoustic pressure wave propagates through the medium, compression and rarefaction ${ }^{1}$ cause loss of acoustic energy. The absorption in underwater acoustic communication increases not only with range, but also with frequency. Frequencydependent attenuation results in a relationship between the communication distance and the highest frequency that can efficiently be used. A short link offers more bandwidth than a long link. Therefore, for a UW-ASN the property holds that by relaying information over multiple hops, the effective bandwidth can be increased significantly.

\footnotetext{
${ }^{1}$ Reduction of density, the opposite of compression.
} 
Compared to the propagation speed of EM waves, the acoustic propagation speed is five orders of magnitude lower. In radio communication, the desired signal and its reflections arrive almost simultaneously; the time interval between the earliest arrival and the latest reflection is, depending on the environment, in the order of microseconds. In underwater communication such an interval can easily exceed tens of milliseconds. Consequently, reflections from current and previous underwater transmissions, also known as multipath components, distort the desired signal being received. The desired signal and the multipath components add up constructively and destructively. Therefore, some frequencies in the (aggregate) received signal are amplified, whereas others are attenuated. This type of distortion is known as frequency-selectivity. Additionally, movement of the surface waves leads to displacement of the reflection points causing propagation paths to change. The latter is termed time-varying multipath propagation and results in time-varying frequency-selectivity.

\subsection{Problem Statement}

To facilitate the growing commercial interest in aquatic monitoring, considerable research effort is initiated. Despite underwater sensor and underwater vehicle technology being relatively mature, underwater communications is still a major challenge [27]. The underwater acoustic channel is often regarded as a communication channel of extreme difficulty.

Time-varying distortion of the received signal, caused by the underwater channel, can be mitigated using spatial and spectral signal processing methods. Most of these techniques can be implemented in terms of filter operations, whose coefficients need to be adapted on the fly. Some of these adaptive methods require nonlinear mathematical functions to be calculated. Additionally, to compensate for nonminimum-phase behavior, it is essential to have support for noncausal filtering. Digital hardware can be programmed to support noncausal adaptive filtering (by introducing lags) and to evaluate nonlinear functions. Nonlinearity and noncausality are particularly cumbersome to deal with in analog hardware. Therefore, in this work, solely digital signal processing (DSP) methods are employed to compensate for the underwater channel distortion.

Underwater communication is expensive in terms of power. For nodes in a UWASN, the required transmission power is typically in the order of tens of watts [79]. The available battery energy of underwater sensor nodes is heavily constrained, because recharging after deployment is often difficult and expensive. Therefore, it is essential to keep transmission time slots as short as possible. Also, to reduce the energy consumption at the receiving underwater nodes, computational capabilities to compensate for the channel distortion are limited. Consequently, the main focus of this thesis is energy-efficient digital spatial and spectral signal processing to provide fast and accurate compensation for the distortion caused by the underwater channel. 


\subsection{APPROACH}

Compensation of channel distortion is called equalization. In radio communications, many conventional equalization methods require training sequences to be transmitted [13]. To reduce energy consumption of the underwater transmitter and to make more efficient use of the transmission time slots, we employ digital blind equalization methods, meaning that we digitally compensate for underwater channel distortion without employing training sequences. As a substitute for the missing training sequences, structural properties of the transmitted signals are exploited.

We have chosen to focus on both (i) blind spatial equalization and (ii) blind spectral equalization techniques. Blind spatial equalization combines signals from different synchronous and spatially separated receivers to create angular regions with high sensitivity to improve reception from a certain direction. In literature, the latter is also known as blind beamforming.

Blind spectral equalization, which can be considered the spectral counterpart of blind spatial equalization, compensates the channel's frequency-selectivity caused by constructive and destructive interference of multipath components.

\subsection{Outline}

In order to mitigate underwater channel distortion, a quantitative analysis of the channel's most distinctive properties is given in chapter 2. To evaluate (blind) equalization techniques in practice, a flexible multi-channel underwater testbed was built. An overview of both its hardware design, as well as its design decisions can be found in chapter 3 . Blind spatial equalization to compensate for the effects of the underwater acoustic channel is discussed in chapter 4. This chapter elaborates on two novel blind methods, the A-CMA and the E-CMA. The topic of chapter 5 is blind spectral equalization of nonminimum-phase channels. Herein, fast and accurate compensation of (first-order) nonminimum-phase channels using the AP-CMA is presented. Finally, in chapter 6, our conclusions and future work are given.

\subsection{Notational CONVEntions}

For the notation of mathematics in this thesis, we adhere to the following rules:

"Scalars are written in normal face lowercase letters, e.g., $x$.

»Vectors are written in boldface lowercase letters, e.g., $\mathbf{x}$.

" Matrices are written in boldface capitals, e.g., $\mathbf{X}$.

Reference units for values expressed in decibels are annotated in subscript. 



\title{
2 \\ THE UNDERWATER CHANNEL
}

\begin{abstract}
In this chapter, the characteristics of the underwater acoustic channel are discussed from the perspective of the most fundamental equation for performance analysis of underwater acoustic communications: the SONAR equation. Since the SONAR equation does not account for sound speed variability, we explain the detrimental effects of sound speed variability on underwater acoustic propagation, by showing ray traces for realistic ocean temperature profiles. No standard deterministic model exists to describe underwater multipath propagation. Therefore, we elaborate on a stochastic model, which is often used in underwater communication literature, the wide-sense stationary uncorrelated scattering (WSSUS) model. In the underwater multipath environment, propagation speed variability can lead to a scenario where the direct-path acoustic pressure wave arrives later than a reflected wave. This phenomenon and its relation to the channel's phase response are elucidated.
\end{abstract}

\subsection{INTRODUCTION}

Following upon the short overview of the underwater acoustic channel in chapter 1, a more quantitative and in-depth discussion of the channel's unique properties is given here. An understanding of these properties is necessary for the design of underwater communication hardware (chapter 3 ) and the development of underwater blind spatial and blind spectral equalization techniques (chapters 4 and 5).

This chapter's quantitative discussion of the underwater channel starts by introducing commonly used terminology and units in section 2.2. Section 2.3 elaborates on the SONAR equation and discusses transmission loss and ambient noise. The variable propagation speed of underwater sound and its effect on acoustic communication is introduced in section 2.4. Section 2.5 discusses deterministic and stochastic models to describe underwater multipath propagation. This section also elaborates on nonminimum-phase behaviour: an important property of (some) underwater channels. The most relevant properties of the underwater channel and their relationship to the subjects in the other chapters can be found in section 2.6.

Parts of this chapter have been published in [KCH:4] . 
This section shortly elaborates on (underwater) acoustics terminology and reference units. Furthermore, it clarifies the difference between the sound pressure level (SPL) and the sound intensity.

\subsubsection{ACOUSTIC PRESSURE WAVES}

Underwater acoustic pressure waves are the main means for communication under water. The standard unit for pressure is pascal $(\mathrm{Pa})$. A pascal is the pressure resulting from a force of 1 newton acting on an area of 1 square meter. In the vicinity of an underwater acoustic source, regions of compression and rarefaction can be distinguished. In a region of compression, the acoustic pressure exceeds the equilibrium condition, whereas in a region of rarefaction, the acoustic pressure is less than the equilibrium condition.

\subsubsection{ACOUSTIC INTENSITY}

The majority of underwater transducers is sensitive to pressure disturbances [66]. Pressure disturbances are converted to voltages and vice versa by means of piezoelectric materials. Although pressure disturbances are measured, often sound intensity (or acoustic intensity) is discussed. Sound intensity is the flow of acoustic energy per unit time through a surface of unit area. Sound intensity is proportional to the sound pressure squared for plane and spherical travelling waves [39]. Therefore, the SPL, which is a scale for the sound pressure squared, is in many cases equal to the sound intensity.

Unique characterization of the SPL, expressed in decibels, requires a reference sound pressure. In underwater acoustics, a reference sound pressure of $1 \mu \mathrm{Pa}$ RMS is commonly used, which is denoted in subscript as $\mathrm{SPL}_{\mathrm{dB}}$ re $1 \mu \mathrm{Pa}$ [1]. Note that the SPL is a ratio of intensities, even though it is referenced to a pressure.

To determine the SPL $L_{\mathrm{dB}}$ re $1 \mu \mathrm{Pa}$ of an acoustic source, the measured RMS pressure $p$ is divided by the reference pressure $p_{\text {ref }}=1 \mu \mathrm{Pa}$ RMS and expressed logarithmically:

$$
\begin{aligned}
\mathrm{SPL}_{\mathrm{dB} \text { re } 1 \mu \mathrm{Pa}} & =10 \log _{10} \frac{p^{2}}{p_{\mathrm{ref}^{2}}} \\
& =20 \log _{10} \frac{p}{p_{\text {ref }}} .
\end{aligned}
$$

In order to develop an intuition for realistic values of underwater sound sources e.g., the $\mathrm{SPL}_{\mathrm{dB} \text { re } 1 \mu \mathrm{Pa}}$ (at $1 \mathrm{~m}$ distance) of a $1 \mathrm{~W}$ omnidirectional sound source is 170.77 dB re $\mu \mathrm{Pa}$ [10]. 
The most fundamental equation for performance analysis of underwater acoustic communication is the passive SONAR equation [34]. This equation can be used to determine the narrowband range- and frequency-dependent signal-to-noise ratio $(\mathrm{SNR})$ available at a receiver, denoted by $\operatorname{SNR}_{\mathrm{dB}}(l, f)$ :

$$
\operatorname{SNR}_{\mathrm{dB}}(l, f)=\mathrm{SL}_{\mathrm{dB}} \text { re } 1 \mu \mathrm{Pa}-\mathrm{TL}_{\mathrm{dB}}(l, f)-\left(\mathrm{NL}_{\mathrm{dB} \text { re } 1 \mu \mathrm{Pa}^{2}}(f)-\mathrm{DI}_{\mathrm{dB}}(f)\right) \text {. }
$$

Herein, $l$ is the range in $\mathrm{km}, f$ the center frequency in $\mathrm{kHz}, \mathrm{SL}_{\mathrm{dB}}$ re $1 \mu \mathrm{Pa}$ the acoustic intensity of the source and $\mathrm{TL}_{\mathrm{dB}}(l, f)$ the transmission loss. Furthermore, $\mathrm{NL}_{\mathrm{dB} \text { re } 1 \mu \mathrm{Pa}^{2}}(f)$ represents the ambient noise level at the receiver and $\mathrm{DI}_{\mathrm{dB}}(f)$ the directivity index of the receiver. Note that the value of $\operatorname{SNR}_{\mathrm{dB}}(l, f)$ is a simplified estimate. E.g., losses caused by fading are not taken into account.

In this work, the acoustic intensity of the source $\mathrm{SL}_{\mathrm{dB}}$ re ${ }_{1 \mu} \mathrm{Pa}$ is expressed using the SPL of the source. By definition, the $\mathrm{SL}_{\mathrm{dB}}$ re $1 \mu \mathrm{Pa}$ is equal to the $\mathrm{SPL}_{\mathrm{dB}}$ re $1 \mu \mathrm{Pa}$ at $1 \mathrm{~m}$ distance. Ambient noise does not require a reference distance, since it does not originate from a single source.

For mathematical tractability, we assume that the $\operatorname{SNR}_{\mathrm{dB}}(l, f)$ is valid for a band of $1 \mathrm{~Hz}$ centered around $f$. To determine the ambient noise level $\mathrm{NL}_{\mathrm{dB} \text { re } 1 \mu \mathrm{Pa}^{2}}(f)$ in such a band, an expression for the channel's ambient noise power spectral density (PSD) can be used, as will be shown in section 2.3.2.

The last term of eq. $2.1\left(\mathrm{DI}_{\mathrm{dB}}(f)\right)$ is the amount by which a receiver rejects omnidirectional noise [34]. If the receiver is omnidirectional and frequency-independent, which is assumed in the remainder of this chapter, then $\mathrm{DI}_{\mathrm{dB}}(f)$ can be set to zero. Array directivity and its relation to the frequency of impinging signals will be discussed in chapter 4 . The remainder of this section elaborates on transmission loss and ambient noise.

\subsubsection{TRANSMISSION LOSS}

As an acoustic pressure wave propagates through the medium, compression and expansion cause loss of acoustic energy. When the acoustic pressure wave expands, the acoustic intensity decreases because acoustic power is spread out over a growing surface area. A spreading factor ( $k$ in eq. 2.2) is used to represent different types of spreading. If the expansion is spherical then the intensity drops quadratically with respect to the distance of the (omnidirectional) acoustic source. The latter is often referred to as spherical spreading and is modeled by $k=2$. In an ocean environment, spherical expansion is limited due to the reflecting ocean bottom and surface. If the bottom and surface act as perfect reflectors, that is with no loss of acoustic energy, then spreading is called cylindrical spreading. The only loss occurs at the area of the 'hull' of the cylinder. Therefore, in case of cylindrical spreading, the acoustic intensity drops linearly with respect to the distance of the acoustic source. Cylindrical spreading is modeled by the spreading factor $k=1$ [28]. 


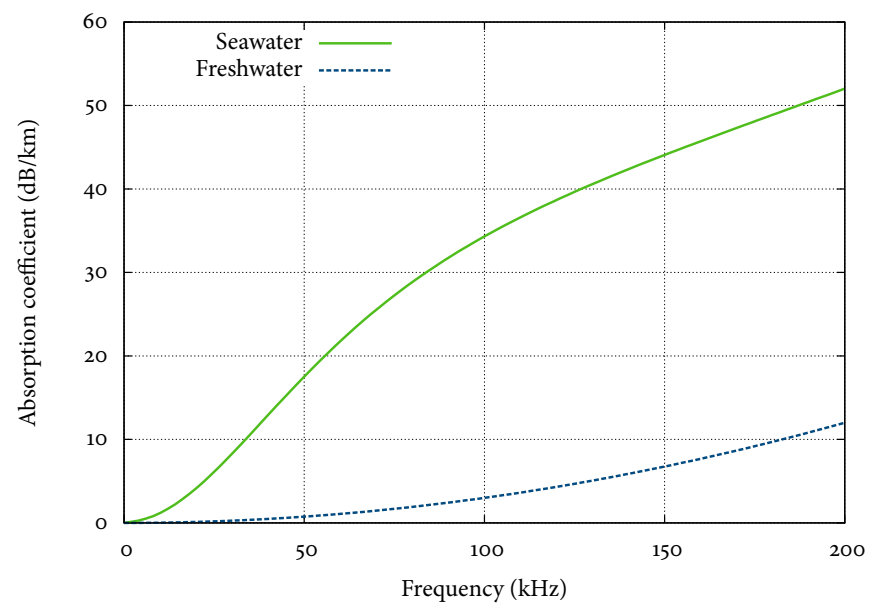

FIGURE 2.1 - Absorption coefficient $a(f)$.

In a realistic setting, pressure wave expansion is often a combination of spherical and cylindrical spreading. To model a combination of both spreading categories, the spreading factor can be chosen accordingly. Often, the value $k=1.5 \mathrm{is}$ used, which is known as practical spreading [78].

The absorption in underwater communication increases not only with range, but also with frequency and (in case of cylindrical spreading) with depth ${ }^{1}$. In general, the transmission loss $\mathrm{TL}_{\mathrm{dB}}(l, f)$ in an underwater acoustic channel over a distance $l$ in $\mathrm{km}$ for a frequency $f$ in $\mathrm{kHz}$ is given by:

$$
\begin{aligned}
\mathrm{TL}_{\mathrm{dB}}(l, f) & =k \cdot 10 \log _{10}\left(\frac{l}{l_{0}}\right)+l \cdot a(f), & & \text { for spherical spr. }(k=2) \\
& =k \cdot 10 \log _{10}\left(\frac{l}{l_{0}}\right)+l \cdot a(f)+\varepsilon, & & \text { for } 1<k<2 \\
& =k \cdot 10 \log _{10}\left(\frac{l}{l_{0}}\right)+l \cdot a(f)+10 \log _{10}\left(\frac{z}{z_{0}}\right) . & & \text { for cyl. spr. }(k=1) \quad(2.2)
\end{aligned}
$$

Herein, $k$ represents the spreading factor, $l_{0}$ a reference distance ${ }^{2}, z_{0}$ a reference depth (in $\mathrm{m}$ ), $z$ the depth (in $\mathrm{m}$ ) and $a(f)$ the frequency-dependent absorption coefficient (in $\mathrm{dB} / \mathrm{km}$ ). Typically, the reference depth $z_{0}$ is set to $2 \mathrm{~m}$. The offset $\varepsilon$, in case the expansion behaves as a combination of both spherical and cylindrical spreading, is a topic for further study within the acoustic community. In the remainder of this thesis, we assume practical spreading with $\varepsilon=0$. However, note that this results in an underestimate of the actual transmission loss.

\footnotetext{
${ }^{1}$ For cylindrical spreading, the dependence on depth can be made explicit by writing $\operatorname{TL}_{\mathrm{dB}}(l, f, z)$.

${ }^{2}$ Typically, this reference distance $l_{0}$ is $1 \times 10^{-3} \mathrm{~km}$.
} 
In freshwater, frequency-dependent attenuation can be explained by taking into account viscous effects of water. However, in seawater the measured losses are much larger than expected from viscous effects alone. For seawater, these additional losses can be explained by the relaxation effects of boric acid and magnesium sulfate. The equation for the absorption coefficient is known as Thorp's equation. Thorp's empirical equation for the absorption coefficient $a(f)($ in $\mathrm{dB} / \mathrm{km}$ ) in seawater can be written as [1]:

$$
a(f)=\left(\frac{0.11 f^{2}}{1+f^{2}}\right)+\left(\frac{44 f^{2}}{4100+f^{2}}\right)+\left(3 \cdot 10^{-4} f^{2}\right) .
$$

Herein, $f$ is the frequency given in $\mathrm{kHz}$. The absorption coefficients (for fresh water and seawater) for frequencies up to $200 \mathrm{kHz}$ are shown in figure 2.1. For (energyefficient) long-range underwater acoustic communication, only the low-frequency range can be exploited. Long-range systems enable communication over distances up to $700 \mathrm{~km}$. Typically, these systems use frequencies in the range of $225 \mathrm{~Hz}$ to $275 \mathrm{~Hz}$ [32]. In this thesis, the focus will be on short- and medium-range communication. Distances up to $20 \mathrm{~km}$ belong to these categories. Chapter 3 discusses choosing appropriate communication frequencies given the characteristics of the underwater channel.

\subsubsection{AMBIENT NOISE LEVEL}

Underwater ambient noise refers to the noise that remains after excluding all easily identifiable sound sources. For example, a nearby ship is treated as an acoustic signal instead of a noise source, although the presence of many ships randomly distributed over the ocean is attributed to ambient noise. Typically, the ambient noise in the underwater channel is caused by (i) turbulence, (ii) shipping, (iii) waves and (iv) thermal noise. The effect of precipitation is not discussed in this section. However, when precipitation is present, it is also an important source of noise [1].

Turbulence and shipping noise are the main noise sources in the low-frequency region $(<100 \mathrm{~Hz})$. Turbulence is low-frequency noise resulting from pressure changes in irregular moving water in turbulent currents [12]. The empirical PSDs of turbulence and shipping noise expressed in $\mu \mathrm{Pa}^{2} \mathrm{~Hz}^{-1}$ are given by [78]:

$$
\begin{aligned}
N_{\mathrm{t}}(f) & =10^{\left(17-30 \log _{10}\left(\frac{f}{f_{\mathrm{r}}}\right)\right) / 10}, \\
N_{\mathrm{S}}\left(f, s_{\mathrm{n}}\right) & =10^{\left(40+20\left(s_{\mathrm{n}}-0.5\right)+26 \log _{10} \frac{f}{f_{\mathrm{r}}}-60 \log _{10}\left(\frac{f}{f_{\mathrm{r}}}+0.03\right)\right) / 10 .}
\end{aligned}
$$

Herein, $f$ is the frequency in $\mathrm{kHz}, s_{\mathrm{n}}$ the shipping factor and $f_{\mathrm{r}}$ the reference frequency, set to $1 \mathrm{~Hz}$. The shipping factor needs to be set in the range of o-1. A smaller factor means less shipping activity. Spatially, the noise intensity of distant shipping is more significant for transmissions parallel to the ocean bottom, because signals impinging on the receiver after multiple bottom reflections will be strongly attenuated [10]. 
The major cause of underwater ambient noise in the region of $100 \mathrm{~Hz}-100 \mathrm{kHz}$, is agitation of the sea surface by wind. In contrast to shipping noise, wind-related noise is more intense in the vertical than in the horizontal plane [10]. The majority of underwater communication systems operate in the $100 \mathrm{~Hz}-100 \mathrm{kHz}$ region. Consequently, wind-related noise strongly affects the performance of these systems. The empirical PSD of wind-related noise in $\mu \mathrm{Pa}^{2} \mathrm{~Hz}^{-1}$ can be written as [78]:

$$
N_{\mathrm{w}}\left(f, w_{\mathrm{n}}\right)=10^{\left(50+7.5 \sqrt{\frac{w_{\mathrm{n}}}{w_{\mathrm{r}}}}+20 \log _{10} \frac{f}{f_{\mathrm{r}}}-40 \log _{10}\left(\frac{f}{f_{\mathrm{r}}}+0.4\right)\right) / 10 .}
$$

Herein, $f$ is the frequency in $\mathrm{kHz}, w_{\mathrm{n}}$ the wind speed in $\mathrm{m} \mathrm{s}^{-1}$ and $w_{\mathrm{r}}$ the reference wind speed, set to $1 \mathrm{~m} \mathrm{~s}^{-1}$. Note that, the (empirical) relationship between wind speed and ambient noise in $\mathrm{dB}_{\mathrm{re}} \mu \mathrm{Pa}^{2} \mathrm{~Hz}^{-1}$ is equal to $7.5 \sqrt{\frac{w_{\mathrm{n}}}{w_{\mathrm{r}}}}$. A very rough approx-

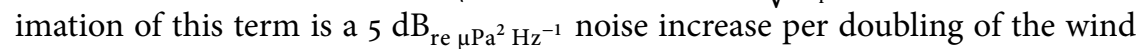
speed.

In the high-frequency region $(>100 \mathrm{kHz}$ ), thermal noise dominates the ambient noise intensity. Thermal noise is the result of random pressure fluctuations (at the transducer) caused by thermally agitated water molecules [1]. The PSD of thermal noise in $\mu \mathrm{Pa}^{2} \mathrm{~Hz}^{-1}$ as function of the frequency $f$ in $\mathrm{kHz}$ is given by [78]:

$$
N_{\text {th }}(f)=10^{\left(-15+20 \log _{10} \frac{f}{f_{\mathrm{r}}}\right) / 10} .
$$

The complete underwater ambient noise spectrum $N\left(f, s_{n}, w_{n}\right)$ in $\mu \mathrm{Pa}^{2} \mathrm{~Hz}^{-1}$ can now be written as:

$$
N\left(f, s_{\mathrm{n}}, w_{\mathrm{n}}\right)=N_{\mathrm{t}}(f)+N_{\mathrm{s}}\left(f, s_{\mathrm{n}}\right)+N_{\mathrm{w}}\left(f, w_{\mathrm{n}}\right)+N_{\mathrm{th}}(f) .
$$

For average Dutch weather conditions and moderate shipping ${ }^{3}$ the PSD of the ambient noise $N\left(f, s_{\mathrm{n}}, w_{\mathrm{n}}\right)$ is shown in figure 2.2. To further illustrate the sensitivity of ambient noise for variations in wind and shipping activity, figure 2.3 shows the ambient noise spectrum for four different combinations of wind and shipping levels.

The PSD for the average Dutch weather and moderate shipping (figure 2.2) roughly decays linearly with respect to the logarithmic abscissa in the region $1 \mathrm{kHz}-100 \mathrm{kHz}$. Similar to the analysis by Stojanovic [78], the following linearization of the empirical PSD can be found:

$$
\begin{aligned}
& \tilde{N}(f)_{\mathrm{dB} \text { re } \mu \mathrm{Pa}^{2} \mathrm{~Hz}^{-1}} \approx N\left(f, s_{\mathrm{n}}, w_{\mathrm{n}}\right)_{\mathrm{dB} \text { re } \mu \mathrm{Pa}^{2} \mathrm{~Hz}^{-1} \mid s_{\mathrm{n}}=0.5, w_{\mathrm{n}}=4.9, f=[1 \ldots 100]} \\
& \tilde{N}(f)_{\mathrm{dB} \text { re } \mu \mathrm{Pa}^{2} \mathrm{~Hz}^{-1}}=62.5-17 \log _{10}\left(\frac{f}{f_{\mathrm{r}}}\right) .
\end{aligned}
$$

In the context of the passive SONAR equation (eq. 2.1), for a bandwidth of $1 \mathrm{~Hz}$

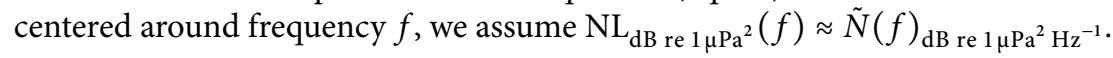

\footnotetext{
${ }^{3} s_{\mathrm{n}}=0.5$ and $w_{\mathrm{n}}=4.9 \mathrm{~m} \mathrm{~s}^{-1}[14,35]$
} 


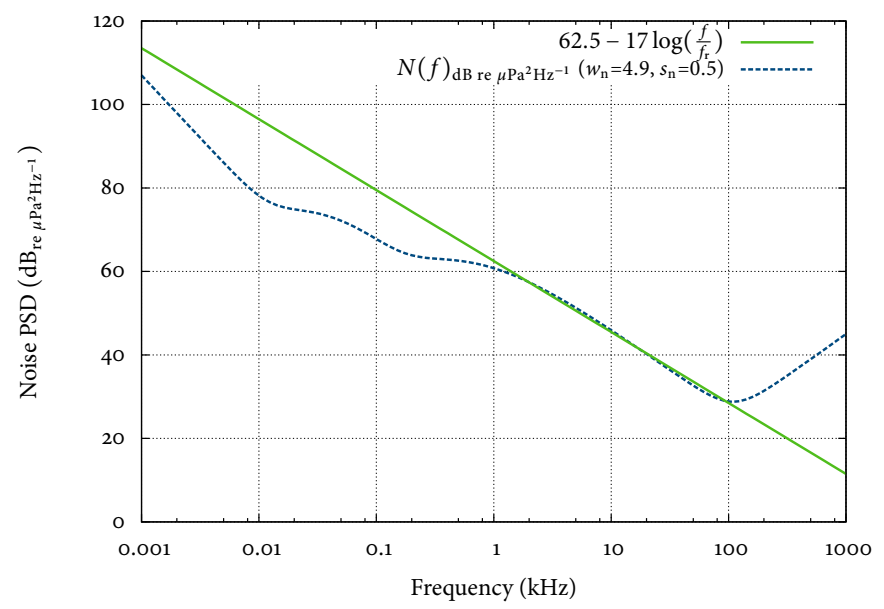

FIGURE 2.2 - Ambient noise PSD and its linearization for the average Dutch wind speed $\left(w_{n}=4.9\right)$ and moderate shipping $\left(s_{n}=0.5\right)$.

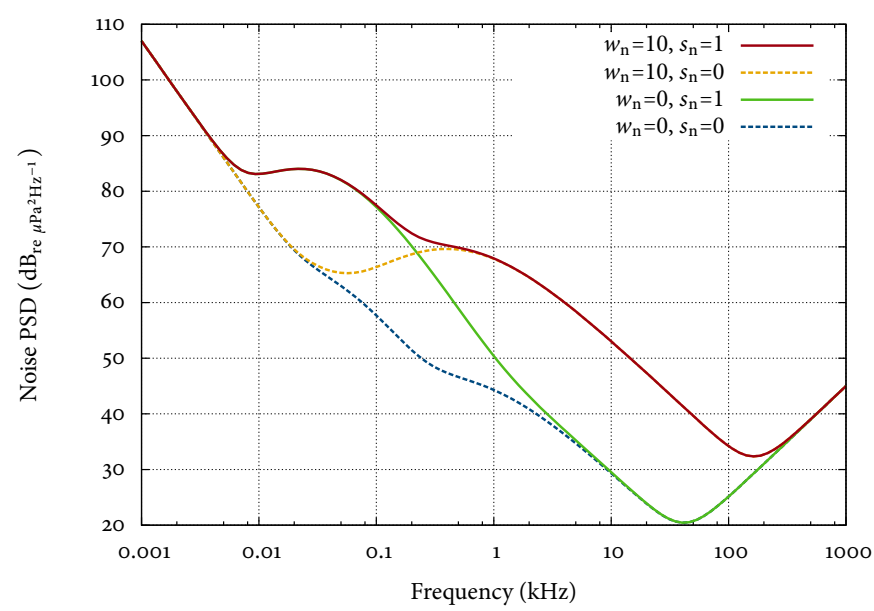

FIGURE 2.3 - Ambient noise PSD for different combinations of wind and shipping levels.

\subsection{VARIABLE PROPAGATION SPEED}

The propagation speed of underwater acoustic pressure disturbances is variable and determined by (i) salinity, (ii) temperature and (iii) depth of the measurement. Temperature and depth are the main factors to influence the speed of sound. Effects of variability in salinity are usually small and often neglected. 
The following equation gives an approximation of the speed of sound $c(\mathrm{~m} / \mathrm{s})$ in a marine environment [46]:

$$
\begin{aligned}
c(S, T, z) & =1448.96+4.591 T-0.05304 T^{2}+2.374 \cdot 10^{-4} T^{3} \\
& +(1.340-0.01025 T)(S-35)+0.01630 z \\
& +1.675 \cdot 10^{-7} z^{2}-7.139 \cdot 10^{-13} \mathrm{Tz}^{3} .
\end{aligned}
$$

Herein, $T$ is the temperature $\left({ }^{\circ} \mathrm{C}\right), S$ the salinity (parts per thousand or ppt) and $z$ the depth (m).

\subsubsection{SOUND SPEED PROFILE}

The diagram of the sound speed as a function of depth $z$ is known as the sound speed profile (SSP). To illustrate sound speed as a function of depth, consider the ocean divided into horizontal layers with different properties. The depth and thickness of these layers heavily depend on the latitude of the ocean region. From surface to bottom, the following ocean layers can be recognized: (i) surface layer, (ii) seasonal thermocline, (iii) main thermocline and the (iv) deep isothermal layer [10]. An underwater thermocline is a region with a rapid decline of temperature with depth.

As an example, in figure 2.4, the temperature and sound speed variation over depth in the Skagerrak region of the North Sea are shown $[4,45]$. The sound speed profiles are approximated using eq. 2.10. The Skagerrak is part of the Norwegian trench and home to the deepest point of the North Sea (700 m).

The main cause of temperature variation (and hence sound speed variation) in the surface layer and the seasonal thermocline is the influence of the sun. Daily

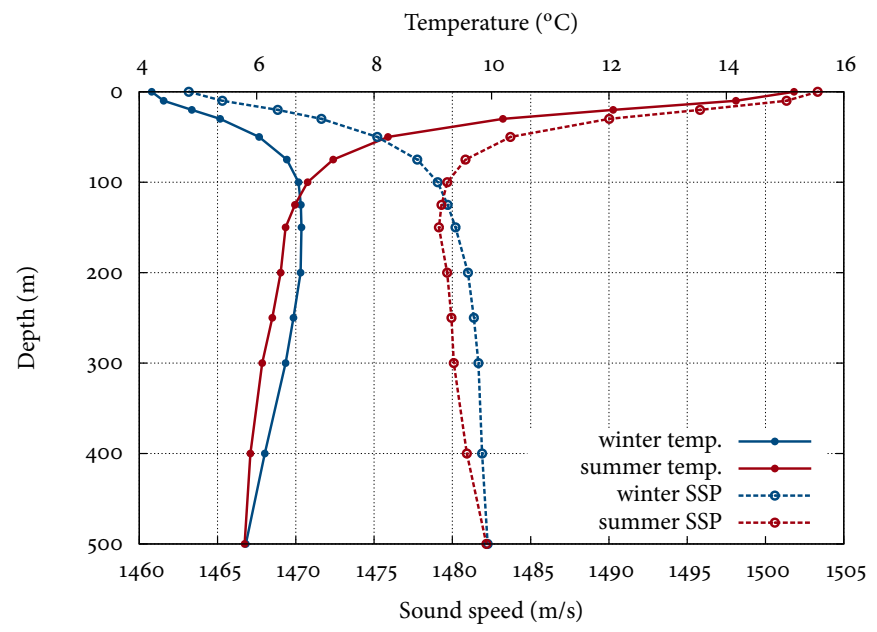

FIgURE 2.4 - Temperature and sound speed profile for the Skagerrak region $\left(57.5^{\circ} \mathrm{N} 8.5^{\circ} \mathrm{E}\right)$. 
temperature fluctuations occur in the surface layer, in the Skagerrak this layer extends to a depth of approximately twenty meters [25]. Furthermore, figure 2.4 clearly reveals the effect of seasonal changes. The layer that fluctuates at a seasonal basis is termed the seasonal thermocline. In this example, the seasonal thermocline extends to a depth of $125 \mathrm{~m}$.

At larger depths, in the main thermocline, the sound speed variation is small because the decrease in temperature is balanced by the increase in depth. If we were able to go even deeper, then at a certain depth the temperature would become constant (isothermal) and the sound speed would only be influenced by depth. According to eq. 2.10, this isothermal layer has a positive sound speed gradient.

In the Skagerrak, the dense deep water does not mix with the less dense surface layer water. This density difference is caused by the low salinity in the upper layer. Formation of layers due to density differences, caused by salinity differences, is called salinity stratification. The low salinity of the upper layer is the result of cold and saltier water, which has a higher density, subducting into the Skagerrak from other parts of the North Sea [44]. Salinity stratification allows the surface layer to become colder (in winter) than the deeper water. In summer, the water layers are also thermally stratified due to the surface layer being heated by the sun.

\subsubsection{SHADOWING}

Sound speed variability has a large effect on underwater acoustic communication because acoustic rays always bend toward regions of decreasing sound speed (Snell's law) [86]. To analyze the effect of ocean sound speed variability, a ray tracer can be employed. A well-known ray tracer for two-dimensional underwater acoustic ray

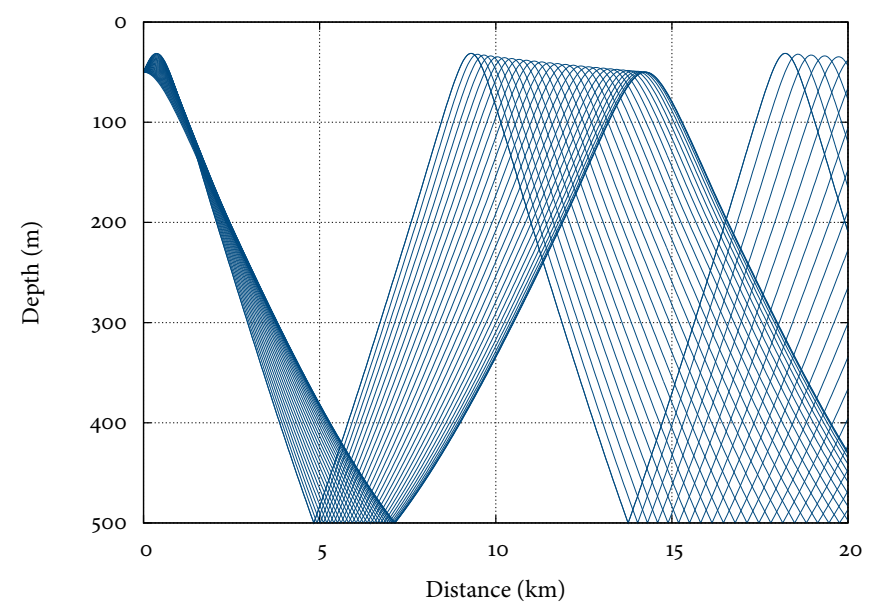

FIGURE 2.5 - Acoustic shadowing in the Skagerrak (in summer). 
tracing is the Bellhop code developed by Porter [62]. Basically, a ray tracer emulates the source by a fan of beams and traces the propagation of these beams through the medium [63]. The pressure or the particle velocity, at a certain location in the medium, is calculated by incorporating the contributions of each individual beam.

The Bellhop code has been executed with the Skagerrak summer and winter profiles (figure 2.4) as input to gather an understanding of the propagation of acoustic energy at these periods of the year. During our simulation with the summer profile, the results of which can be seen in figure 2.5, the position of the acoustic source is $50 \mathrm{~m}$ below the ocean surface. The ocean bottom is located at a depth of $500 \mathrm{~m}$. A source with a highly directional fan of beams was used to emphasize the effects of sound speed variability being illustrated. In summer, the surface layer and seasonal thermocline have a negative sound speed gradient, causing rays to bend downward. A region can be recognized with zero acoustic intensity, which is called the acoustic shadow zone. Until the boundary of this shadow zone, the transmission loss can be accurately approximated using spherical spreading (spreading factor $k=2$ ) [10].

Based on SSP data measured during winter months (figure 2.4), another set of ray traces has been calculated. Results of this simulation are shown in figure 2.6. In winter, the positive sound speed gradient in both the surface layer and the seasonal thermocline causes rays to bend upward and to become trapped near the water surface. This phenomenon is called surface ducting [1]. If all the transmitted acoustic energy gets confined in a surface duct then the transmission loss can be approximated using cylindrical spreading (spreading factor $k=1$ ) [10].

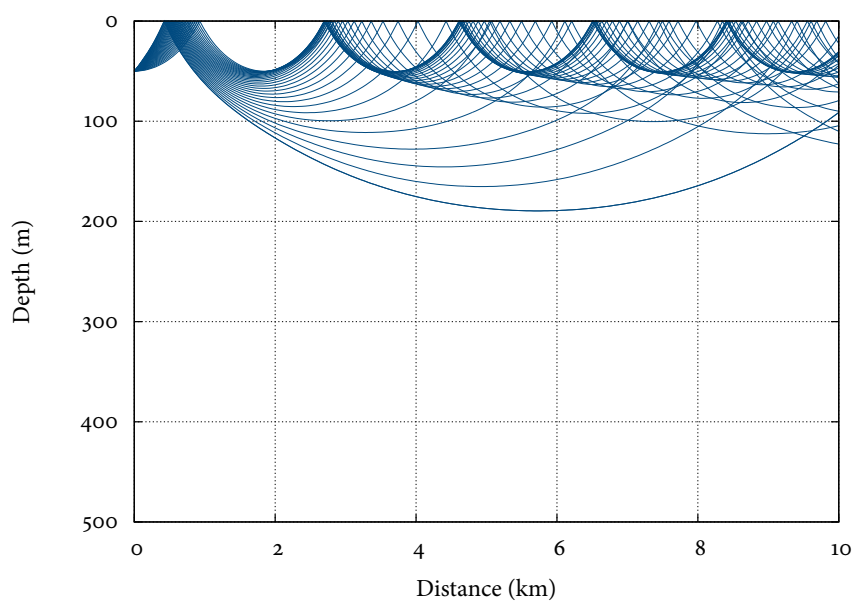

FIGURE 2.6 - Surface ducting in the Skagerrak (in winter). 


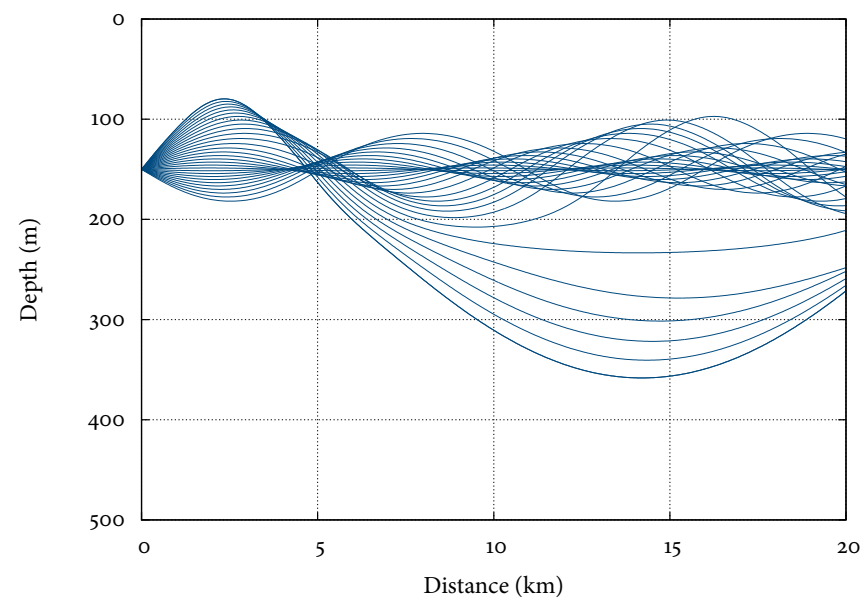

FIGURE 2.7 - Formation of an acoustic waveguide in the Skagerrak (in summer).

\subsubsection{ACOUSTIC WAVEGUIDE}

In summer, the Skagerrak has a minimum sound speed at a depth of approximately $150 \mathrm{~m}$ (figure 2.4). An acoustic source with a nearly horizontal directivity positioned in the Skagerrak (at $150 \mathrm{~m}$ of depth) is visualized in figure 2.7. Herein, the presence of an acoustic waveguide can easily be recognized. An acoustic waveguide is formed by acoustic rays oscillating across the axis of the sound speed minimum and it results in an acoustic intensity that diminishes in a cylindrical fashion. Whether or not this characteristic is truly cylindrical is determined by the directivity of the source and the SSP of the channel.

The SOFAR channel, which was mentioned in chapter 1, is a seasonally independent waveguide that permits underwater acoustic waves to travel over great distances. The axis of the SOFAR channel is close to the water surface at high latitudes, but deepest in subtropic regions [17]. Typically, the depth of this channel is $1 \mathrm{~km}$ [1].

\subsection{Multipath propagation}

The complex propagation of underwater acoustic energy, originating from a single source, has been discussed in sections 2.4.2 and 2.4.3. We have seen that an underwater receiver encounters ocean regions with zero acoustic intensity due to shadowing, as well as multipath-rich regions with a large intensity. Instead of merely focusing on acoustic intensity, this section elaborates on characterizing the (time-varying) superposition of signal reflections experienced in a multipath-rich underwater environment. In addition to characterization of time-varying multipath, an important property of (some) multipath underwater channels, known as nonminimum-phase behavior, is discussed. 
In a completely time-invariant environment, under the assumption that the received signal is a linear combination of the line-of-sight (LoS) component and/or time-delayed attenuated reflections of the source signal $s(t)$, the received complex baseband signal $x(t)$ can be written as follows:

$$
x(t)=\sum_{p=1}^{P} h_{p} s\left(t-\tau_{p}\right) .
$$

Herein $h_{p}$ and $\tau_{p}$ represent, respectively, the complex gain factor and time delay of the $p^{\text {th }}$ propagation path. Often, this equation is generalized as follows to cover for a continuum of all possible time delays $\tau$ :

$$
x(t)=\int_{-\infty}^{\infty} h(\tau) s(t-\tau) d \tau .
$$

The function $h(\tau)$ is called the impulse response of the well-known linear timeinvariant (LTI) channel model.

\subsubsection{DETERMINISTIC TIME-VARYING MULTIPATH MODEL}

The objects reflecting the source signal are known as scatterers. In a realistic environment, the scatterers, as well as the acoustic source, the medium and the receiver can be moving. Clearly, the assumption of time-invariance does not hold in such a practical environment. Therefore, linear time-variant (LTV) channel models were developed. The remainder of this section elaborates on LTV channel modeling.

Movement results in Doppler frequency shifts of the source signal. If all propagation paths and their respective Doppler shifts are completely known, a deterministic linear description of the received complex baseband signal $x(t)$, in terms of the source signal $s(t)$, can be given as follows [29]:

$$
x(t)=\sum_{p=1}^{P} h_{p} s\left(t-\tau_{p}\right) e^{j 2 \pi v_{p} t} .
$$

Herein, $h_{p}, \tau_{p}$ and $v_{p}$ are, respectively, the complex gain factor, time delay and Doppler shift of the $p^{\text {th }}$ propagation path.

Equation 2.13 can be generalized to describe a continuum of propagation paths. For every possible time delay $\tau$ and Doppler shift $v$, a complex gain factor $S_{h}(\tau, v)$ is defined [5]:

$$
x(t)=\int_{-\infty}^{\infty} \int_{-\infty}^{\infty} S_{h}(\tau, v) s(t-\tau) e^{j 2 \pi v t} d \tau d v
$$

Function $S_{h}(\tau, v)$ is called the delay-Doppler spreading function, since it describes the spreading of the source signal in time and frequency. 
Apart from the delay-Doppler spreading function, an LTV channel is often characterized in terms of its time-varying impulse response $h(t, \tau)$. Note that, compared to the LTI impulse response $h(\tau)$ of eq. 2.12, the dependence on time is modeled by incorporating the observation time $t$.

The time-varying impulse response $h(t, \tau)$ can be found by integrating out the Doppler shift of the spreading function $S_{h}(\tau, v)$ :

$$
h(t, \tau)=\int_{-\infty}^{\infty} S_{h}(\tau, v) e^{j 2 \pi v t} d v
$$

At first glance, the multiple notions of time may seem confusing. Therefore, keep in mind that the physical notion of $h(t, \tau)$ is the response of the time-varying channel at observation time $t$ for an impulse at time $t-\tau$.

\subsubsection{STOCHASTIC TIME-VARYING MULTIPATH MODEL}

In practice, it will never be possible to give a complete deterministic characterization of an underwater multipath channel. Usually, stochastic characterizations based on stochastic process theory are employed. Stochastic processes are indexed sequences of stochastic variables. Each stochastic variable assigns a probability to the outcome of a random experiment. A stochastic channel characterization describes the statistical properties of an ensemble of possible channel realizations. The objective of a stochastic characterization is to cover as much of the channel dynamics as possible while still being mathematically and computationally tractable.

A commonly used assumption to simplify the modeling of underwater acoustic LTV channels is the wide-sense stationary uncorrelated scattering (WSSUS) assumption [18]. WSSUS channel models are discussed in the following section.

\section{Wide-sense stationary uncorrelated scattering assumption}

Under the assumption that the received signal consists of a large number of diffusely reflected multipath components, often $h(t, \tau)$ is assumed to be a two-dimensional zero-mean complex Gaussian process (Central Limit Theorem) [29]. In literature, a stochastic process defined over a parameter space with a dimensionality of at least one is referred to as a random field. For example, an Euclidean random field is an infinite set of stochastic variables indexed in Euclidean space.

The zero-mean Gaussian random field $h(t, \tau)$ representing an LTV channel can completely be characterized by its autocorrelation function $\gamma_{h h}\left(t_{1}, t_{2}, \tau_{1}, \tau_{2}\right)$ [49]:

$$
\gamma_{h h}\left(t_{1}, t_{2}, \tau_{1}, \tau_{2}\right)=h\left(t_{1}, \tau_{1}\right) h^{*}\left(t_{2}, \tau_{2}\right) p\left(h\left(t_{1}, \tau_{1}\right), h\left(t_{2}, \tau_{2}\right)\right)=E\left\{h\left(t_{1}, \tau_{1}\right) h^{*}\left(t_{2}, \tau_{2}\right)\right\} .
$$

Herein, $p\left(h\left(t_{1}, \tau_{1}\right), h\left(t_{2}, \tau_{2}\right)\right)$ is the joint probability density function (PDF) which represents the chance of $h\left(t_{1}, \tau_{1}\right)$ and $h\left(t_{2}, \tau_{2}\right)$ occurring simultaneously. The asterisk denotes complex conjugation. Clearly, eq. 2.16 is not practical since it 
depends on four variables and the joint PDF $p\left(h\left(t_{1}, \tau_{1}\right), h\left(t_{2}, \tau_{2}\right)\right)$ needs to be known.

In [5], Bello introduced the WSSUS condition that can be used to reduce the autocorrelation function $\gamma_{h h}\left(t_{1}, t_{2}, \tau_{1}, \tau_{2}\right)$ of an LTV channel to an expression in two variables. The first step of Bello's simplification is to assume that the channel $h(t, \tau)$ is wide-sense stationary (WSS). For a WSS channel, the first- and second-order statistics are independent of absolute time. The autocorrelation function $\gamma_{h h}$ is a second-order statistic, therefore the following holds:

$$
\gamma_{h h}\left(t_{1}, t_{2}, \tau_{1}, \tau_{2}\right)_{\mid \text {Wss }}=\gamma_{h h}\left(t_{1}+t^{\prime}, t_{2}+t^{\prime}, \tau_{1}, \tau_{2}\right) \quad \forall t^{\prime} \in \mathbb{R} .
$$

This implies that this autocorrelation function, under the WSS assumption, only depends on the time difference between $t_{1}$ and $t_{2}$, denoted by $\Delta t=t_{1}-t_{2}$ :

$$
\gamma_{h h}\left(t_{1}, t_{2}, \tau_{1}, \tau_{2}\right)_{\mid \mathrm{WSS}}=\gamma_{h h}\left(\Delta t, \tau_{1}, \tau_{2}\right)
$$

Bello's second simplification of $\gamma_{h h}$ incorporates the assumption that the channel's delay coefficients corresponding to paths with different delays are uncorrelated. Application of this uncorrelated scattering (US) property results in:

$$
\gamma_{h h}\left(t_{1}, t_{2}, \tau_{1}, \tau_{2}\right)_{\mid \mathrm{US}}=\gamma_{h h}\left(t_{1}, t_{2}, \tau_{1}, \tau_{2}\right) \delta\left(\tau_{1}-\tau_{2}\right) .
$$

Herein, $\delta$ represents the Dirac delta function. The US property implies that a single delay $\tau=\tau_{1}=\tau_{2}$ is sufficient to represent the delay coefficient for $\gamma_{h h}$ :

$$
\gamma_{h h}\left(t_{1}, t_{2}, \tau_{1}, \tau_{2}\right)_{\mid \mathrm{US}}=\gamma_{h h}\left(t_{1}, t_{2}, \tau\right) \text {. }
$$

A combination of the WSS and US condition yields the WSSUS condition that reduces the autocorrelation function of an LTV channel $h(t, \tau)$ to an expression in two variables:

$$
\gamma_{h h}\left(t_{1}, t_{2}, \tau_{1}, \tau_{2}\right)_{\mid \mathrm{WSSUS}}=\gamma_{h h}(\Delta t, \tau) .
$$

\subsubsection{NONMINIMUM-PHASE BEHAVIOUR}

In the underwater multipath environment, the variation in acoustic propagation speed can lead to a scenario where the direct-path acoustic pressure wave arrives later than a reflected wave. In section 1.3, we already mentioned this channel property, known as nonminimum-phase behaviour. As its name suggests, the minimumphase or nonminimum-phase property of a channel is related to the phase behaviour of the channel. A (causal) channel $h$ with frequency response $H(\omega)$ (for normalized frequencies) is minimum-phase if its phase change between $H(\pi)$ and $H(0)$ is equal to zero [64]:

$$
\arg [H(\pi)]-\arg [H(0)]=0 .
$$

Whether a channel satisfies the minimum-phase or nonminimum-phase property is of vast importance, since it is closely related to the channel's invertibility and identifiability. Both of these properties are crucial with respect to mitigation of channel 


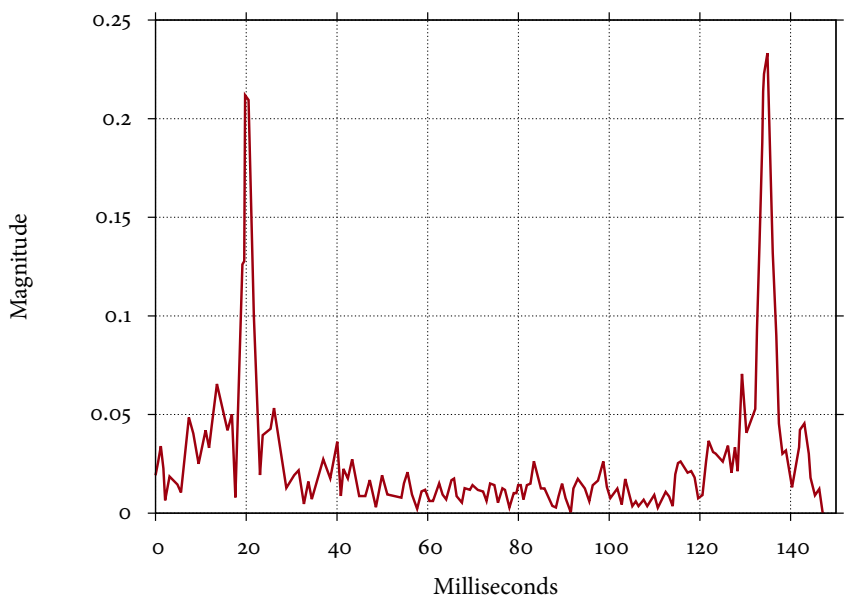

FIGURE 2.8 - Empirical channel response illustrating nonminimum-phase behaviour [18].

distortion. Invertibility and identifiability of (non)minimum-phase channels are discussed in chapter 5 and appendix A, respectively.

An actual example of an empirical underwater channel response ${ }^{4}$ can be seen in figure 2.8. In this (extremely) sparse response, the second arrival has a larger amplitude than the first arrival. As a rule of thumb, an impulse response having most of its energy concentrated near the start of the response is (often) a minimum-phase response. Since the response in figure 2.8 does not comply to this rule of thumb, it can be considered a nonminimum-phase channel.

To our opinion, a channel modeled by $h(t, \tau)$, under the assumption that $h(t, \tau)$ is a Gaussian random field, cannot express nonminimum-phase behaviour. Our assumption of this non-expressibility is based on the fact that $h(t, \tau)$ is fully characterizable by its autocorrelation function (eq. 2.16) and for its time-invariant analog $h(\tau)$ such a characterization always results in a minimum-phase system (chapter 5). (Possible) non-expressibility of nonmininum-phase behaviour should be taken into account during channel sounding and its subsequent channel characterization.

\subsection{CONCLUSIONS}

Quantitative channel characterization, based on the SONAR equation, reveals that the ambient noise limits the lowest frequency that can efficiently be used for communication. Ambient noise levels are variable; a major cause of this variability is agitation of the sea surface by wind. Consequently, wind conditions strongly affect the performance of underwater communication systems. On the other end, the

\footnotetext{
${ }^{4}$ Figure is reproduced with permission of the publisher.
} 
upper frequency limit is a result of underwater absorption not only increasing with range, but also with frequency. Design decisions during development of our underwater acoustic testbed, related to the aforementioned characteristics, are discussed in chapter 3 .

The underwater sound speed is variable and determined by salinity, temperature and depth. Based on empirical ocean temperature profiles, ocean sound speed profiles were calculated. The ocean's sound speed variability has a large effect on underwater acoustic communication, since acoustic rays always bend toward regions of decreasing sound speed. Using an acoustic ray tracer, based on our empirical sound speed profiles, we illustrate (i) rays bending downward, (ii) rays bending upward and (iii) rays travelling across a horizontal axis. In literature, these effects are respectively known as acoustic shadowing, surface ducting and formation of an acoustic waveguide.

Apart from merely focusing on acoustic intensity, the channel can also be mathematically characterized as a time-varying superposition of the direct-path signal and its reflections. Such an LTV response is useful to determine the received signal for every possible input signal. However, in practice, it is never possible to give a complete deterministic LTV characterization. Therefore, a (simplified) stochastic characterization based on the WSSUS assumptions was discussed.

By characterizing the channel as a superposition of a direct-path signal and its reflections, an important channel property can be recognized: the variation of acoustic propagation speed can lead to the scenario where the direct-path pressure wave arrives later than the reflection(s). This nonminimum-phase behaviour is related to the invertibility and identifiability of a channel. Chapter 5 elaborates on compensating for signal distortion caused by nonminimum-phase channels. 


\title{
3 \\ MULTI-CHANNEL UNDERWATER \\ TESTBED
}

\begin{abstract}
Current systems for (multi-channel) underwater signal processing suffer from a tight relation between the hardware and physical layer software. To provide a low-cost, small form factor and flexible solution, this chapter presents a (receive-only) multi-channel testbed consisting of an off-the-shelf FPGA board and a simple expansion board. Nevertheless, the proposed testbed provides the flexibility and processing power to evaluate novel physical layer algorithms in real-life experiments. Two underwater experiments have been performed to verify the functionality of the testbed and to gather measurement data.
\end{abstract}

\subsection{INTRODUCTION}

In the previous chapter we discussed the challenging characteristics of the underwater channel. To compensate for the signal distortion caused by the channel, different physical layer techniques in time, frequency and/or space can be exploited. To evaluate existing and novel physical layer techniques, a flexible hardware setup is required.

This chapter presents an underwater testbed consisting of an (up to eight-element) array connected to an off-the-shelf field programmable gate array (FPGA) board via an easy to build extension board. The use of a readily available FPGA board reduces the amount of engineering work before any practical underwater experiment can be performed. Nonetheless, the testbed provides enough flexibility to evaluate different types of equalization and modulation techniques.

This chapter is organized as follows. Related multi-channel systems for underwater acoustic processing are discussed in section 3.2. A short description of the requirements is presented in section 3.3. System-level design decisions during the design of the testbed are enforced by properties of the underwater channel and beamforming theory. The system-level design is discussed in section 3.4. An overview of the

Parts of this chapter have been published in [KCH:4] . 
system implementation is given in section 3.5. Underwater experiments were performed to verify the functionality of the testbed. These experiments are discussed in section 3.6. An overview of the most significant results and directions for future work can be found in section 3.7.

\subsection{Related WORK}

In literature, two other systems that are related to our multi-channel testbed were identified: the Woods Hole Oceanographic Institution Micro-modem and the reconfigurable modem (rModem) from the Massachusetts Institute of Technology [20] [76]. Although both systems are capable of multi-channel signal reception, they are primarily designed to provide a means for rapid testing and development of algorithms beyond the physical layer of the open systems interconnection (OSI) stack.

\subsubsection{MICRO-MODEM}

The Micro-modem is an autonomous modem which can be used to build underwater (wireless) communication networks [58]. Apart from communication, the modem also contains a subsystem for navigation.

In its basic configuration, the Micro-modem uses frequency-hopping frequencyshift keying (FH-FSK) modulation for acoustic communication. FH-FSK is binary frequency-shift keying (FSK) where the modulator hops through (predetermined) orthogonal frequencies and modulates data on a different frequency at each hop [58]. FH-FSK modulation provides robust, but low data rate communication. For example, the default data rate in the FH-FSK mode is $80 \mathrm{bit} \mathrm{s}^{-1}$. Transmissions use $4 \mathrm{kHz}$ of bandwidth and can be performed at different carrier frequencies (10, 15 and $25 \mathrm{kHz}$ ). Frequency hopping serves two purposes: (i) multi-user communication and (ii) inter-symbol interference (ISI) mitigation. In a multi-user environment, different hopping patterns allow different users to transmit simultaneously. In a single-user application, ISI decreases, because frequency hopping allows reflections, at a particular frequency, to decrease in power before information is modulated on that frequency again.

The main processing board of the Micro-modem is based around a fixed-point DSP. The board's analog input and output are connected to a 12-bit analog-to-digital converter (ADC) and a 12-bit digital-to-analog converter (DAC). For phase-shift keying (PSK) based communication, (class D) amplification, as well as multi-channel signal reception, separate expansion boards are available. All Micro-modems can transmit PSK modulated signals, but only mainboards with the PSK expansion board can receive these transmissions. The data rates in the PSK mode are in the range of 300 to $5000 \mathrm{bit} \mathrm{s}^{-1}$.

The modem communicates with the user via a standard serial connection based on the National Marine Electronics Association specification (version 0183). The 
disadvantage of the Micro-modem is the tight relation between the physical level software implementation and the hardware itself. Implementation of other types

of physical layer algorithms would require changes to proprietary firmware.

\subsubsection{RECONFIGURABLE MODEM}

The tight relation between physical layer software and hardware in existing off-theshelf modems triggered MIT to design their own acoustic modem. MIT's acoustic modem, termed rModem, is a small form factor mainboard that uses both an FPGA and a floating-point DSP for processing [76]. Since the rModem is mainly a research tool, the relatively large power consumption of its components is tolerated.

For interfacing the analog hardware, the rModem contains four 16-bit ADCs and four 16-bit DACs. The expansion board interface contains the power amplifier board, which is connected to transducer(s) operating in the $9-14 \mathrm{kHz}$ frequency range. The FPGA is meant for intermediate frequency (IF) processing, such as filtering, interpolation and decimation. The demodulator, data link layer and network control layer are implemented on the DSP. To relax coding requirements, a large SDRAM and flash memory are available on the board.

Although the rModem would be a good alternative for the Micro-modem, it is not available off-the-shelf.

\subsection{REQUIREMENTS}

Because of the disadvantages stated in section 3.2, none of the existing boards could be used. Therefore, we decided to develop our own testbed. The requirements for the testbed are rooted in (i) functionality, (ii) flexibility and (iii) availability needs.

In SeaSTAR, a coarse-grained monitoring architecture consisting of multiple sensor nodes operating in an area of two square kilometer is envisioned. Being able to communicate over this area's worst case distance $(2 \sqrt{2} \mathrm{~km})$ is an important functional design requirement for the testbed.

The multi-channel testbed should be practical in different types of underwater experiments. Both spectral and spatial equalizers, as well as sensor network algorithms need to be tested. Verification of spatial equalization techniques requires multiple transducers to be synchronously sampled and to be positioned in an array configuration. Practical experiments where the individual transducers conduct as (receive-only) sensor nodes in a sensor network do not require synchronous sampling. Therefore, flexibility in terms of transducer configuration and processing capabilities is needed.

Because the firmware of the Micro-modem is proprietary and the rModem cannot be bought; to guarantee availability, we decided to develop our own hardware. To reduce development time, principal components of our testbed need to be available off-the-shelf. 


\subsubsection{CHOICE OF OPERATING BANDWIDTH AND TRANSDUCER}

An important property of the aforementioned characteristics of underwater communication channels is the frequency-dependent attenuation (section 2.3.1). This property results in a dependence between the desired distance and the upper frequency limit of the useful acoustic bandwidth. We consider the useful acoustic bandwidth as the frequency range that can be used efficiently, in terms of power, by acoustic communication systems. The lower frequency limit of the useful bandwidth is the consequence of colored ambient noise.

In [78], Stojanovic discusses the relationship between communication distance and the optimum frequency for which the maximum narrowband SNR is obtained. Using this analysis, the useful bandwidth for our testbed can be determined. Recall the narrowband range- and frequency-dependent $\operatorname{SNR}_{\mathrm{dB}}(l, f)$, stated in eq. 2.1, and assume a unit source level $\left(\mathrm{SL}_{\mathrm{dB}}\right.$ re $\left.1 \mu \mathrm{Pa}=0\right)$ and frequency-independent directionality $\left(\mathrm{DI}_{\mathrm{dB}}(f)=0\right)$ :

$$
\operatorname{SNR}_{\mathrm{dB}}(l, f)=-\mathrm{TL}_{\mathrm{dB}}(l, f)-\mathrm{NL}_{\mathrm{dB} \text { re } 1 \mu \mathrm{Pa}^{2}}(f) .
$$

Based on the transmission loss model for practical spreading (eq. 2.2) and our approximation of the narrowband ambient noise level (eq. 2.9), a closed-form expression for the narrowband SNR is given as:

$$
\operatorname{SNR}_{\mathrm{dB}}(l, f)=-\left(k \cdot 10 \log _{10}\left(\frac{l}{l_{0}}\right)+l \cdot a(f)\right)-\left(62.5-17 \log _{10}(f)\right) .
$$

Note that $a(f)$ is Thorp's empirical equation for the frequency-dependent attenuation (eq. 2.3).

Given a reference distance $l_{0}$ of $1 \mathrm{~m}$ and the expansion loss set to practical spreading $(k=1.5), \operatorname{SNR}_{\mathrm{dB}}(l, f)$ is visualized in figure 3.1. Clearly, the effect of the frequencydependent attenuation can be recognized. The larger the communication range, the smaller the useful acoustic bandwidth becomes. To determine the expected SNR value at a receiver, as a figure of merit, recall that the $S P L_{d B}$ re $1 \mu \mathrm{Pa}$ (at $1 \mathrm{~m}$ distance) of a $1 \mathrm{~W}$ omnidirectional sound source is $170.77 \mathrm{~dB}_{\text {re } \mu \mathrm{Pa}}[10]$.

Our envisioned course-grained monitoring architecture should be able to operate in an area of two square kilometer. The worst-case distance in such an area, between a single pair of nodes, is $2 \sqrt{2} \mathrm{~km}$. In a realistic deployment, multiple nodes will be used. Therefore the typical communication distance will be much smaller. Figure 3.2 shows the underwater acoustic range- and frequency-dependent $\operatorname{SNR}_{\mathrm{dB}}(l, f)$ for $0.5,1$ and $2 \sqrt{2} \mathrm{~km}$.

To convert acoustic energy into electrical energy (and vice versa), electroacoustic transducers are used. Underwater electroacoustic transducers can be receive-only (hydrophones), transmit-only (projectors) or a combination of both (transceiving 
transducers) [85]. To comply with our flexibility requirement, transceiving transducers are chosen instead of separate projectors and hydrophones. Based on the assumption that it is likely to have communication distances smaller than $2 \sqrt{2} \mathrm{~km}$, the relatively low-cost and off-the-shelf available HAARI ${ }^{1}$ WBT-3o transducer was chosen, which has an operating frequency starting at approximately $20 \mathrm{kHz}$ and a flat frequency response in the $20 \mathrm{kHz}$ to $40 \mathrm{kHz}$ region.

\footnotetext{
${ }^{1}$ Hangzhou Applied Acoustics Research Institute
}

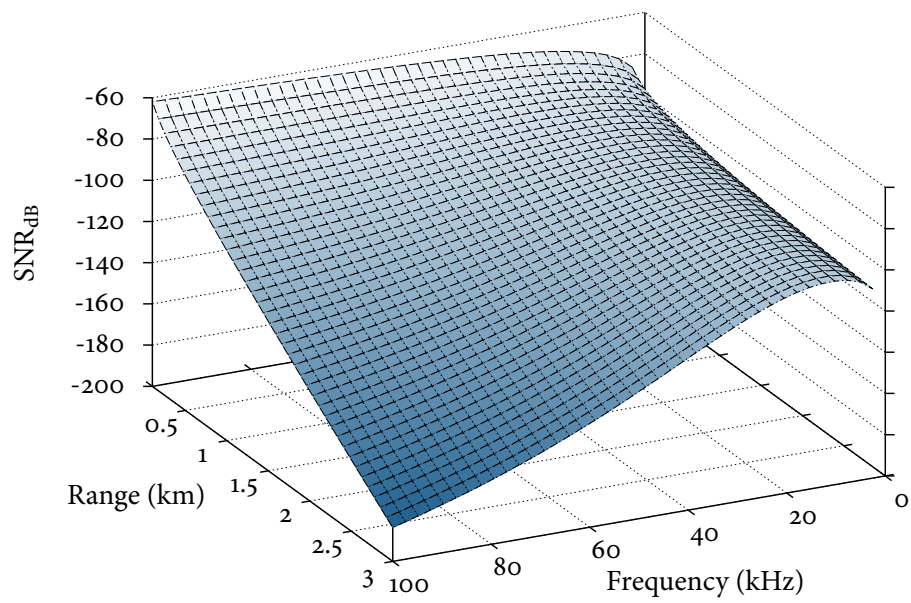

FigURE 3.1 - Narrowband $\operatorname{SNR}_{\mathrm{dB}}(l, f)$.

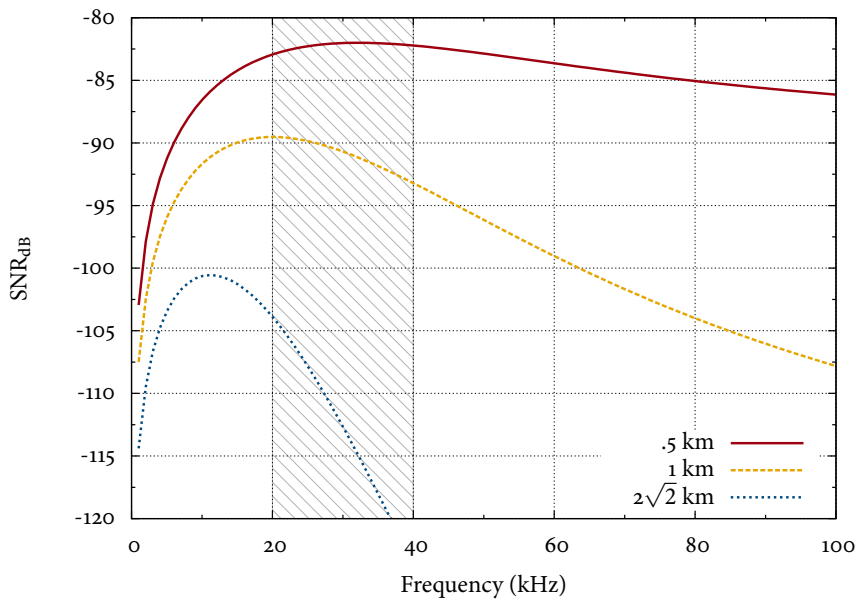

FIgURE 3.2 - Narrowband $\operatorname{SNR}_{\mathrm{dB}}(l, f)$ for $0.5,1$ and $2 \sqrt{2} \mathrm{~km}$. The passband of the WBT-30 transducer $(20 \mathrm{kHz}$ to $40 \mathrm{kHz})$ is annotated. 
To collect samples, an array of up to eight transducers is used. Coherent summing of these samples results in an angular directionality of the array. The directionality of the array can be steered electronically. Creation of these angular regions of directionality is a type of spatial equalization, known as beamforming. Beamforming will be discussed extensively in chapter 4 . A purpose of the multi-channel testbed is to evaluate beamforming algorithms that adapt to changing signal (conditions and) directions. These algorithms are known as adaptive beamforming algorithms.

Characteristics of the underwater channel and the transducer affect design decisions related to beamforming. The next sections clarify the implications of these characteristics.

\section{Wideband nature}

The type of processing that needs to be performed for beamforming depends on the bandwidth of the received signal with respect to its carrier frequency. The latter relationship is known as the fractional bandwidth (FB) and can be written as [2]:

$$
\mathrm{FB}=\frac{f_{h}-f_{l}}{\left(f_{h}+f_{l}\right) / 2} \cdot 100 \% .
$$

Herein, $f_{h}$ and $f_{l}$ are, respectively, the highest and the lowest frequency components in the desired signal. A signal is considered narrowband if the FB is one percent or less [3]. A narrowband signal can be approximated as a sinusoidal signal. Therefore, in a narrowband beamformer, phase shifts can be used to compensate for time lags (caused by non-broadside arrivals) in order to provide coherent summing of the transducer signals. Note that, time lags experienced due to multipath propagation can often not be corrected by mere phase shifts.

In underwater communications, the acoustic bandwidth of signals is (often) in the order of its carrier frequency. For example, if the complete passband bandwidth of the WBT-3o transducer is used for communication, then the FB of the transceived signal is $\frac{40-20}{(40+20) / 2} \cdot 100 \% \approx 67 \%$. A signal having a FB larger than one percent is called a wideband signal. Wideband signals cannot be treated as sinusoidal signals and consequently the performance of narrowband beamformers starts to deteriorate for wideband signals. A variety of techniques can be used to implement wideband beamforming, e.g., delay-sum beamformers, interpolating beamformers and tapped delay line (TDL) based methods [43].

TDL based beamformers sample the impinging signals in both time and space by appending multiple time delays to each antenna [36]. Their structure can be regarded as a finite impulse response (FIR) filter for each antenna. Our research focuses on these TDL based beamformers, because their structure offers a lot of flexibility. Typically, a TDL structure uses a large number of multipliers. The FPGA in our testbed offers flexibility and tens of embedded multipliers to perform TDL based processing. 
The spacing between elements of an array $d$ (in $\mathrm{m}$ ) is generally expressed in terms of fractions of the wavelength $\lambda$ (in $\mathrm{m}$ ) of the highest frequency $f_{h}$ (in $\mathrm{Hz}$ ) in the received signal. Ideally, wideband equidistantly spaced beamformers have $d \leq \frac{1}{2} \lambda$ [43].

The passband of the WBT-30 transducer extends from $20 \mathrm{kHz}$ upto $40 \mathrm{kHz}$. Due to the relatively large diameter of the WBT-30 transducer $(44 \mathrm{~mm})$ the array cannot be ideally spaced, since $\frac{1}{2} \lambda$ for a $40 \mathrm{kHz}$ underwater acoustic transmission is approximately $18.75 \mathrm{~mm}$. The actual array spacing in the testbed is shown in figure 3.3 .

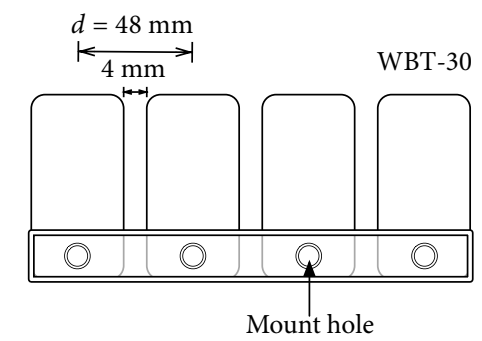

FIgURE 3.3 - Linear array made of four WBT-30 transducers.

A non-ideal spacing of isotropic array elements $\left(d>\frac{1}{2} \lambda\right)$ leads to spatial aliasing, which can be recognized as grating lobes in the array pattern. Based on the average underwater acoustic propagation speed $\left(1500 \mathrm{~m} \mathrm{~s}^{-1}\right)$ and the array spacing of $48 \mathrm{~mm}$, the highest frequency without grating lobes is $15.625 \mathrm{kHz}$. To illustrate these grating lobes, the magnitude of the array response is evaluated as function of frequency and angle of arrival in figure 3.4. For impinging signals with wavelengths equal to or smaller than the array spacing $(\lambda=0.048 \mathrm{~m})$, complete grating lobes are unavoidable. Complete grating lobes are grating lobes with a magnitude equal to the main beam's magnitude.

The grating lobes are a serious complication because the flat frequency response of the transducer commences at $20 \mathrm{kHz}$. Fortunately, by limiting the angular coverage (maximum angle of arrival) of the array and/or the highest frequency $f_{h}$ in the received signal, the impact of complete grating lobes can be reduced. The maximum steering angle $\theta_{0}$ before a complete grating lobe appears at $\theta_{g}= \pm 90^{\circ}$ follows from [3]:

$$
\begin{aligned}
\pi \frac{d}{\lambda}\left[\sin \theta_{g}-\sin \theta_{0}\right] & = \pm n \pi \quad(\text { find first alias, use } n=1) \\
{\left[\sin \theta_{g}-\sin \theta_{0}\right] } & = \pm \frac{\lambda}{d} \quad\left(\text { use } \theta_{g}= \pm 90^{\circ}\right) \\
\theta_{0} & =\sin ^{-1}\left( \pm \frac{\lambda}{d} \pm 1\right) .
\end{aligned}
$$


If a signal with $f_{h}$ equal to $20 \mathrm{kHz}(\lambda=0.075 \mathrm{~m})$ impinges at the current setup, Equation 3.6 has real solutions for $\theta_{0}= \pm 34^{\circ}$. Thus, by limiting the angular coverage to $\left|\theta_{0}\right|<34^{\circ}$ (for this scenario) complete grating lobes do not appear.

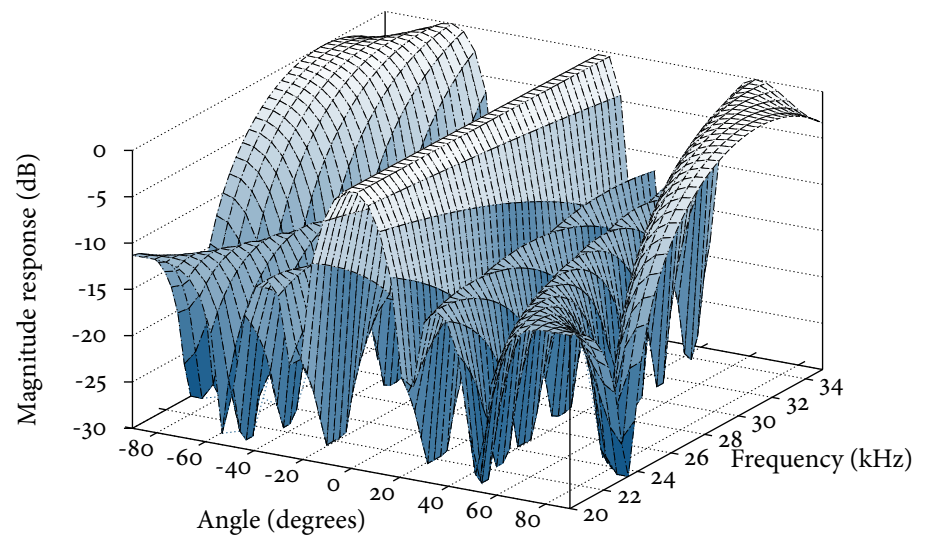

FIGURE 3.4 - Theoretical WBT-30 array response.

\subsubsection{PROCESSING PLATFORM}

In contrast to the Micro-modem and the rModem, our testbed uses an off-the-shelf FPGA mainboard. The use of a readily available FPGA board reduces the amount of engineering work before practical experiments can be performed. Our testbed is by no means an autonomous multi-channel modem. However, it provides a lot of flexibility in terms of processing and enables full control over the raw datastreams from the transducers. To test novel physical layer algorithms in real-time or offline, flexibility and access to raw data of all individual transducers can be regarded as a necessity.

\subsection{SySTEM IMPLEMENTATION}

A simplified schematic of the implementation of our multi-channel underwater testbed can be seen in figure 3.5. From left to right: an array of piezoelectric underwater transducers (figure 3.3) converts acoustic pressure disturbances to voltages. After amplification and low-pass filtering of these voltages, all analog signals are sampled synchronously and copied to the on-board memory of the FPGA board.

A brief description of the electronic hardware of our testbed is presented in this section. At the time of this writing, Terasic has a small form factor, low-cost development kit available with a relatively large FPGA and SDRAM memory (DE0Nano) [82]. To reduce engineering work, only a simple expansion board was added to fully constitute the electronics of our testbed. The analog operations for data acquisition (amplification, filtering and sampling) are performed on the expansion 


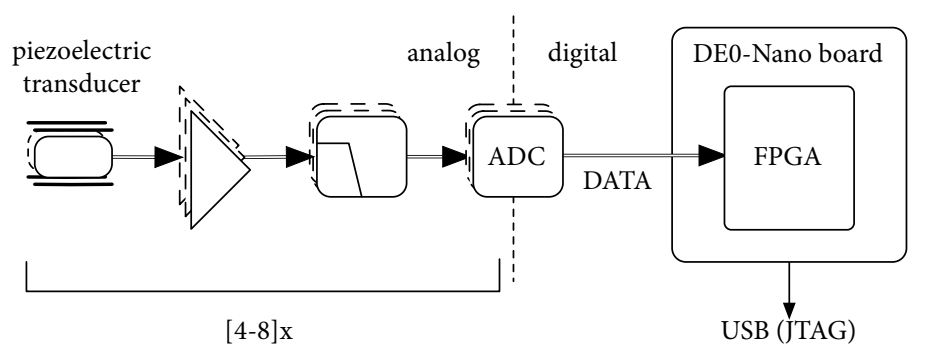

FiguRE 3.5 - Overview of the multi-channel underwater testbed.

board. All other operations are carried out in the digital domain on the FPGA. Figure 3.6 shows our expansion board stacked on top of the DE0-Nano. Upcoming sections discuss the major components of the expansion board and the two systemon-chip (SoC) architectures that have been built for physical layer processing on the FPGA.

\section{Expansion board hardware}

The multi-channel expansion board physically connects to four transducers. Two expansion boards can be stacked to capture upto eight channels. The WBT-30 transducer consists of piezoelectric ceramics encapsulated in a polyurethane housing. These piezoelectric ceramics generate electrical charges proportional to underwater pressure disturbances. Typically output voltages of the transducers are small and preamplifiers are needed. Initially, the electronics are positioned above the water surface and connected by long cables $(20 \mathrm{~m})$ to the transducers in the water. Because of these long cables charge mode amplifiers are used. A charge mode amplifier

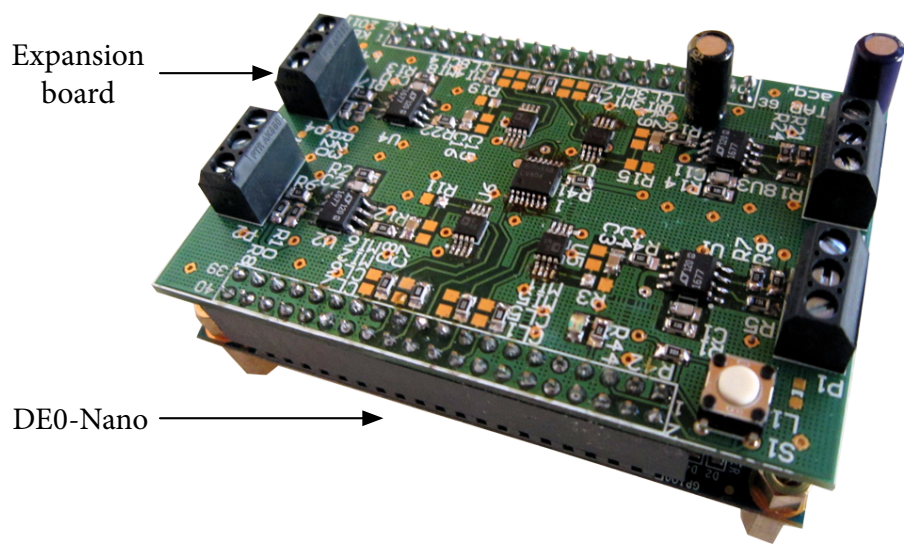

Figure 3.6 - Multi-channel expansion board stacked on top of the DEo-Nano. 
is a high gain operational amplifier with a feedback capacitor. The advantage of this configuration is that the output voltage is independent of the cable capacitance and other parasitic capacitances [40].

After amplification, first-order anti-alias filters are used before the data is synchronously sampled by 12-bit ADCs. The sampling speed of these converters is set to $150 \mathrm{kHz}$. However, these ADCs have a maximum sampling frequency of $3 \mathrm{MHz}$. Therefore, if necessary, oversampling can be applied to improve resolution.

Data is transferred from the ADCs to the FPGA via serial peripheral interface (SPI) buses. The input signal of the ADCs is sampled at the falling edge of the activelow chip select $(\overline{\mathrm{CS}})$. To guarantee synchronous sampling, a single $\overline{\mathrm{CS}}$ signal is generated on the FPGA and branched to all ADCs on the expansion board(s).

\subsubsection{MEMORY-MAPPED SYSTEM-ON-CHIP ARCHITECTURE}

During the first practical experiments, the SoC in figure 3.7 was instantiated on the FPGA. In this configuration, raw sample data from the ADCs is copied to the SDRAM on the DE0-Nano board and stored for further processing. To increase comprehensibility, the eight synchronization clock signals which are part of the SPI buses are not shown. The SoC uses off-the-shelf IP cores that communicate via a memory-mapped bus. Therefore, this SoC can be constructed and programmed with little engineering effort.

The $\overline{\mathrm{CS}}$ output that branches to all ADCs is generated in the FPGA by combining the $\overline{\mathrm{CS}}_{1 \ldots 8}$ signals using an AND gate. The SPI controllers are sequentially activated by software running on the Nios II softcore processor. After activation, $\mathrm{SPI}_{1}$ assigns $\overline{\mathrm{CS}}_{1}$ a logic low, which results in a logic low $\overline{\mathrm{CS}}$ output. The falling edge of this $\overline{\mathrm{CS}}$ output initiates simultaneous sampling of all ADCs. Due to the sequential activation of the SPI controllers and overlap of the corresponding data transfers, $\overline{\mathrm{CS}}$ stays low until the sample data from all ADCs is read.

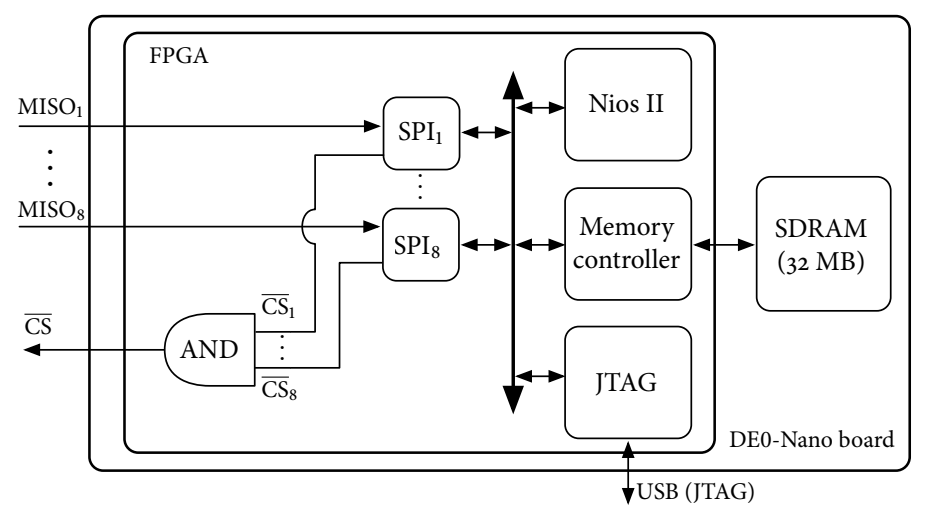

FIGURE 3.7 - SoC for (synchronous) multi-channel data acquistion and storage. 
Raw data is copied to the SDRAM until the $32 \mathrm{MB}$ memory limit is reached. In the memory-mapped SoC architecture, JTAG over USB is used to transfer data from the SDRAM to the PC.

\subsubsection{STREAMING SYSTEM-ON-CHIP ARCHITECTURE}

For raw data acquisition, the memory-mapped architecture (figure 3.7) offers an easy to build solution. Nevertheless, finite buffer sizes, low sample rates and the low JTAG transfer speed are a bottleneck for more involved experiments. Therefore, a streaming SoC architecture has been built that supports (i) real-time signal processing and (ii) real-time streaming of the (preprocessed) transducer signals to a PC.

The streaming SoC architecture for the multi-channel testbed can be seen in figure 3.8. This architecture is referred to as a streaming SoC architecture, because the data path for the sample data is implemented using only streaming interfaces. The source of the sample data is the ADC interface block, which acts as an SPI master for all ADCs and provides for synchronous sampling. The eight streams of raw sample data, a single stream per transducer, are input to the DSP block. The DSP block is a placeholder for real-time signal processing operations, such as mixing and filtering. Internally, the DSP block enables branching of its eight input channels to a maximum of 64 output channels. Whether all of these output channels are actually in use depends on the type of processing being performed in the DSP block. Eventually, the data is sent to the USB client interface that provides USB communication with the client PC.

Compared to the memory-mapped architecture (figure 3.7), the control and data path have been completely separated. To configure the sample rate, to set filter coefficients and to control communication with the PC; the ADC interface, the DSP block and the USB client interface are connected to the memory-mapped bus. Separation of the control and data path enables higher data throughputs, since the parallel nature of the FPGA can now be exploited.

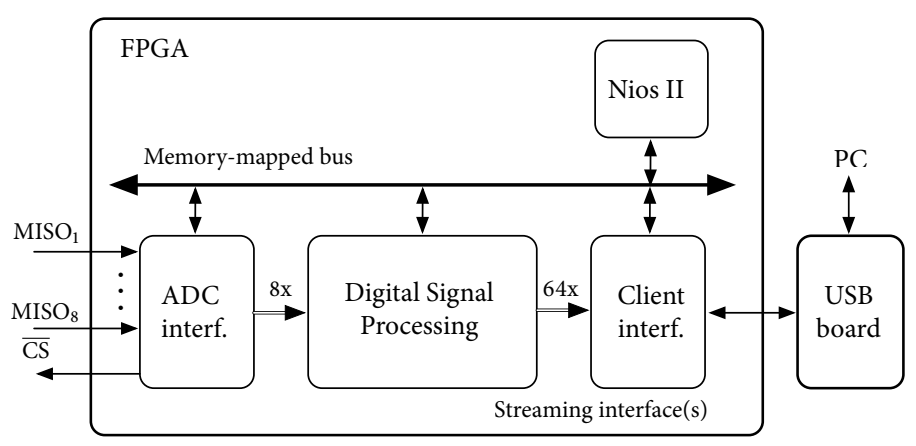

FIGURE 3.8 - SoC for streaming multi-channel data processing. 
The multi-channel underwater testbed (figure 3.5) has been used in two underwater experiments. Brief descriptions of these experiments and their outcomes are given below. The array terminology employed in this section is introduced in section 4.2.

\subsubsection{POOL EXPERIMENT}

In November 2011, underwater experiments were performed in a (freshwater) swimming pool at SUASIS Underwater Systems in Kocaeli, Turkey. The main purpose of these experiments was to verify the functionality of the testbed and to determine the array pattern of the multi-channel receiver.

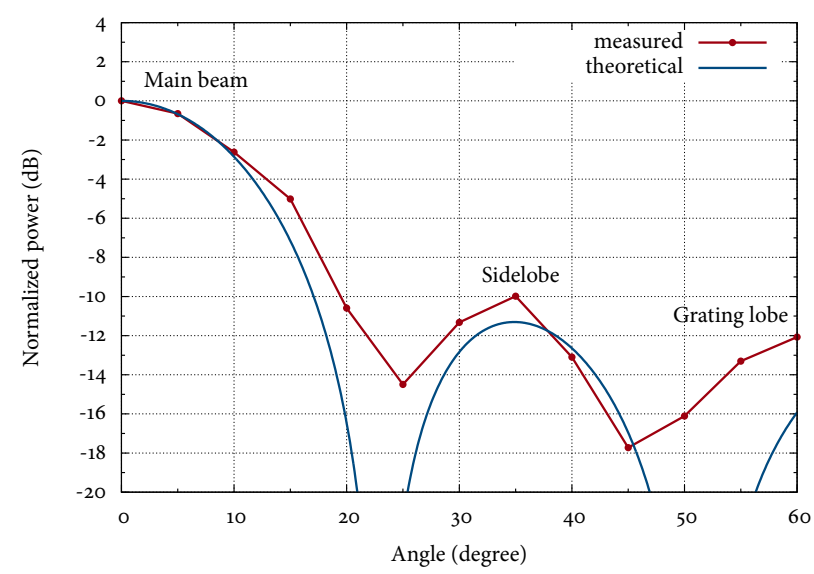

FIGURE 3.9 - Theoretical and measured four-channel array pattern.

An array of four WBT-30 transducers (figure 3.3) was placed in the pool and connected by long cables $(20 \mathrm{~m})$ to the multi-channel expansion board. Another transducer was placed in the far-field of the array and connected via a power amplifier to a signal generator producing short bursts (3 cycles) of a $20 \mathrm{kHz}$ sinusoid.

For twelve different angles, starting from broadside, sample data of a burst was stored in the SDRAM of the DE0-Nano. Afterwards, for every angle, the power of the beamformer output is determined by summing the four channels and taking the mean-square of this sum.

The normalized results of our experiment and the expected theoretical array pattern are shown in figure 3.9. The main lobe, sidelobe and grating lobe of the measured array pattern can clearly be recognized. Furthermore, a measured sidelobe level of $-10 \mathrm{~dB}$ is close to the theoretically expected level. We assume that small displacements of the individual array elements are an important source of the differences between the theoretical and empirical results. 


\subsubsection{DIVE-CENTER EXPERIMENT}

To gather data for evaluation of (i) beamforming techniques and (ii) to analyze performance of localization methods in a practical setting, experiments have been performed in a ten meter deep water tank. A graphical overview of the setup is presented in figure 3.10 and a photograph is shown in figure 3.11. During these experiments, the array of four WBT-30 transducers (figure 3.3) was attached to a stainless steel frame and located at the bottom of the tank. Acoustic signals were transmitted from four WBT-30 transducers $\left(\mathrm{TX}_{1}, \ldots, \mathrm{TX}_{4}\right)$ attached to a buoyant tube floating half a meter below the water surface. The transmissions were captured by the four-element array and recorded by the testbed located above water. Long cables $(20 \mathrm{~m})$ were used to connect the transducers of the array to the testbed's data acquisition board.

\section{Array processing experiment}

During two tests, empirical data for evaluation of array processing techniques has been collected. This section shortly elaborates on these measurements.

In the first test, acoustic sweep signals $(20-40 \mathrm{kHz})$ were consecutively transmitted by the four reference transducers. Decomposing the received wideband sweep
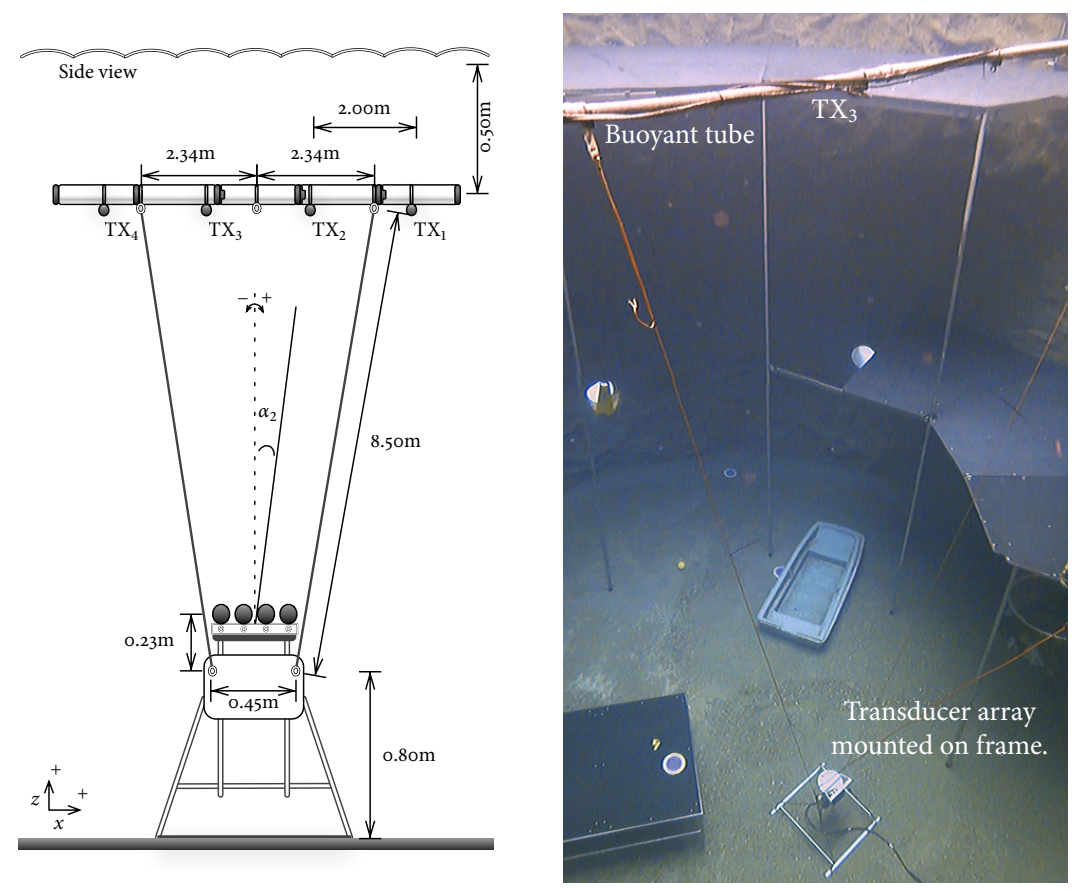

Figure 3.10 - Technical sketch of the setup. Figure 3.11 - Panorama of the test setup. 
into multiple narrow subbands validates the use of narrowband array processing techniques to create directionality. For each of the four reference transmissions, phase shift based beamforming (section 4.2.5) in the $20-20.5 \mathrm{kHz}$ subband, has been used to determine the signal power for every angle. These results are known as beamscan estimates [51] and are shown in figure 3.12. Multiple delayed copies of the original sweep signal reach the array from different directions. For each beamscan, the angle corresponding to the strongest signal power is considered the empirical LoS arrival angle. The expected LoS arrival angles, based on the geometry of the setup (figure 3.10), are indicated by the black triangles. Since our setup did not incorporate a ground-truth reference, it is difficult to justify the deviations between the empirical and the expected LoS angles. Nevertheless, for all four transmissions, this deviation is much less than the half-power beamwidth (HPBW) (section 4.2.7).

The second test focused on multi-channel reception of modulated signals. During this test, a narrowband quadrature phase-shift keying (QPSK) signal (40o bit s ${ }^{-1}$ ) was transmitted by reference transducer $\mathrm{TX}_{2}$. The multi-channel recording of this reference transmission has been used for empirical evaluation of various beamforming methods.

To qualitatively demonstrate the extent of the multipath experienced in the water tank, a scatter plot of the QPSK symbols at the output of the beamformer is shown in figure 3.13. For generation of this plot, the beamformer was configured to steer the main beam in the direction of the empirical LoS arrival angle associated with
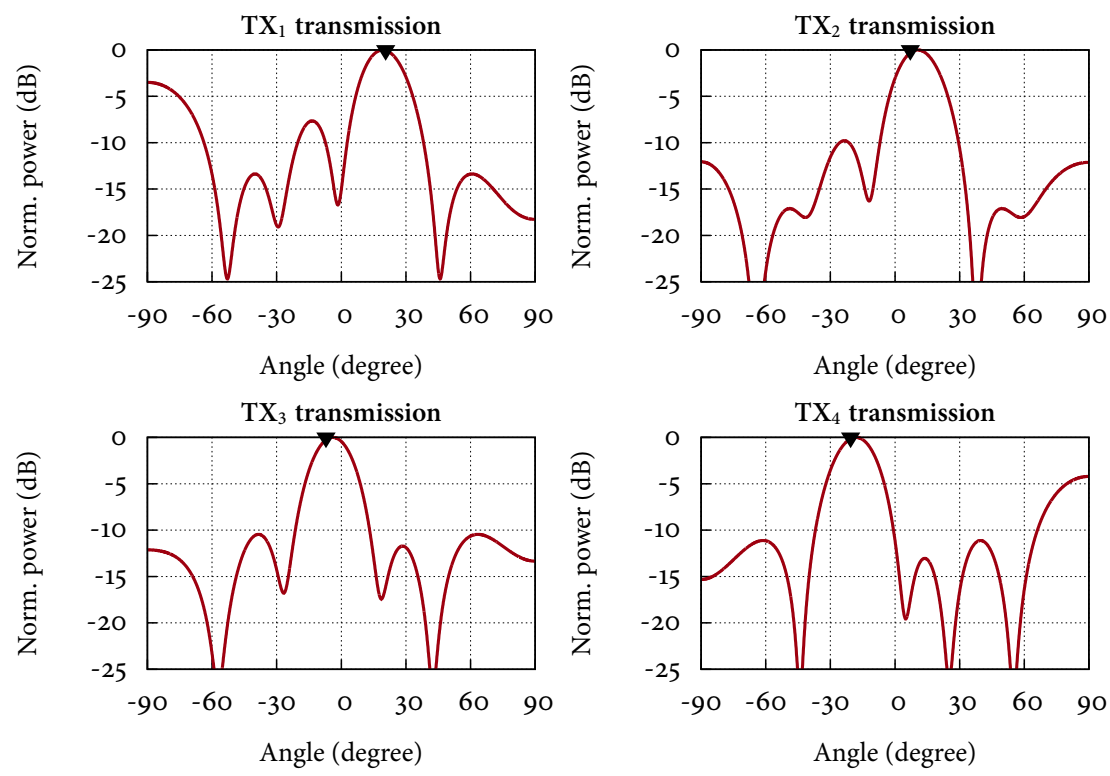

FIGURE 3.12 - Beamscan estimates for the reference transmissions. 


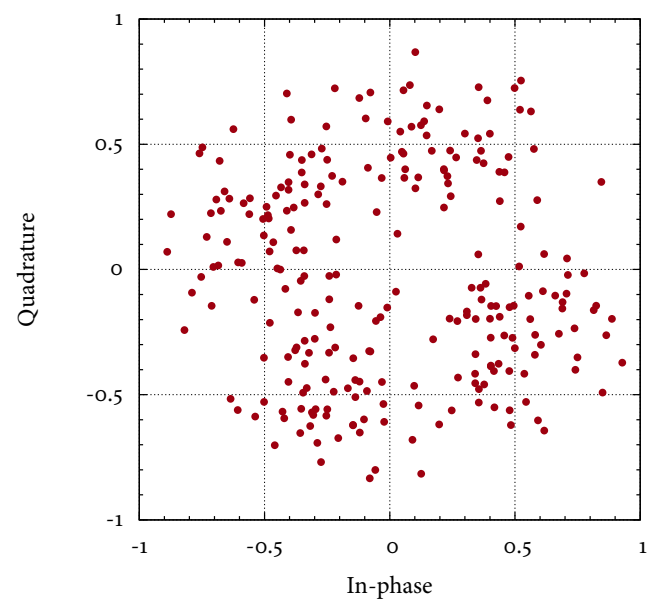

FIGURE 3.13 - Scatter plot after beamforming in the direction of the strongest signal power.

$\operatorname{TX}_{2}\left(\theta=10.6^{\circ}\right)$. In the constellation, four point clouds corresponding to the QPSK symbols can be recognized. Nevertheless, by adapting the directionality of the array more intelligently, the beamformer output can be further improved. In chapter 4, we elaborate on methods to adapt the directionality of an array without the use of training data. The empirical data gathered in this experiment is used to demonstrate the effectiveness of this approach.

\section{Localization experiment}

For underwater nodes in a UW-ASN, it is crucial for every node to know its position with respect to the other nodes [15]. Accurate underwater localization is a challenging task. Although localization is not the topic of this thesis, in the divecenter experiment, our testbed has also been employed to evaluate localization techniques. The availability of a four-element array made it possible to evaluate localization techniques that combine direction-of-arrival (DoA) and time-of-flight (ToF) information to perform localization and time-synchronization. An extensive description of this localization experiment can be found in [ $\mathrm{KCH}: 5]$.

\subsection{Conclusions}

An underwater acoustic testbed for evaluation of novel multi-channel physical layer algorithms is presented in this chapter. Based on the requirements and the physical limitations of the underwater channel, the appropriate transducer was chosen. To provide a low-cost, small form factor and flexible solution, an off-the-shelf FPGA board and a simple expansion board are used to interface with the transducers. On 
the FPGA, a SoC of readily available IP components was instantiated to provide (synchronous) data acquisition, storage and retrieval.

In underwater experiments, the array pattern of the testbed was measured and modulated transmissions were recorded. The measured array pattern resembles the exact pattern with sidelobe levels close to the theoretical levels. The collected measurements are used for evaluation of (blind) array processing techniques in chapter 4 . 


\title{
SPATIAL EQUALIZATION
}

\begin{abstract}
Aвstract - The spatial dimension can be considered for mitigation of underwater channel distortion. Linear combinations of spatially distinct samples, whose coefficients are known as weights, are used to improve the signal-of-interest. Instead of training sequences, the constant modulus property of the transmitted signal is exploited to find appropriate weights. A well-known method that uses this property for weight calculation is the constant modulus algorithm (CMA).

This chapter introduces two methods that both exploit the constant modulus property for weight calculation: (i) the extended CMA (E-CMA) and (ii) the angular CMA (A-CMA). The E-CMA is an algorithm that changes the directionality of a receiver, while simultaneously correcting for phase offsets. For our empirical data set, the E-CMA exhibits promising performance. In contrast to other methods, the A-CMA optimizes the steering angle instead of the array weights. Effectively, this dimensionality reduction results in faster convergence and a lower MSE floor.
\end{abstract}

\subsection{INTRODUCTION}

To compensate for the distortion of the underwater channel (chapter 2), different signal processing techniques can be employed. A signal being transmitted through a wireless channel, not necessarily an underwater channel, can be specified in terms of time, space and frequency. Time, space and frequency are the primary dimensions of the channel and the transmitted signal [11]. An ideal transmission occupies only a designated subspace within time-space-frequency. However, in practice unwanted energy from other subspaces often enters the signal's subspace. An example of these spillovers is spatial interference: spillovers of interferers overlapping the desired signal in the space dimension.

Mitigation of signal energy spillovers, beyond the intended time-space-frequency signal subspace, has to take place in the same time-space-frequency playground. In this chapter, for the most part, the spatial dimension is considered for mitigation

Parts of this chapter have been published in $[\mathrm{KCH}: 1,2]$. 
of signal distortion, which is known as spatial equalization. Spatial equalization is processing performed on snapshots of samples captured at the same time instant and the same frequency, but on different locations in space.

In literature, often the terms spatial equalization and array processing are used ambiguously. However, not all forms of array processing can be regarded as purely spatial equalization. In general terms, array processing can be defined as spatiotemporal equalization. Spatio-temporal equalization refers to processing of samples captured at multiple instants of time at multiple locations in space. Spatial equalization is a type of array processing. In this chapter, we prefer to use the general term array processing. From the context it will be clear whether we allude to spatial equalization or, e.g., spatio-temporal equalization.

A linear combination of spatially distinct samples captured by an array of transducers results in directionality of the array. Directionality is a preference for certain directions over others. The coefficients used to weigh the signals from the individual array elements are called array weights. Ideally, array directionality enhances the source signal, while attenuating noise and interferers. A concise introduction to the theory and terminology of arrays and array processing is given in section 4.2.

For array processing in dynamic environments, such as the underwater channel, the array weights need to be continuously adjusted to compensate for the changing signal (conditions and) directions. Adaptive methods are required to adequately update these weights. A short overview of adaptive array processing techniques can be found in section 4.3 .

An important subclass of adaptive array processing techniques is the class of blind adaptive array processing techniques. These blind methods use structural properties of the transmitted signal to calculate appropriate weight adjustments. Compared to non-blind equalization methods, this approach has several advantages. Since training sequences are not required, the scarce capacity of the underwater channel is more efficiently used. Furthermore, shortening transmission time slots by dropping training sequences reduces the energy consumption of underwater acoustic transmitters. Also, shorter transmissions decrease the risk of collisions in multipoint networks, such as UW-ASNs [52].

To attain relatively high data rates, while keeping the transmission time slots short, efficient use of the available bandwidth is necessary. To achieve (relatively) high spectral efficiencies, while being power-efficient, our underwater transmissions are assumed to be QPSK modulated. QPSK signals have an interesting structural property for blind adaptive array methods to exploit, namely, the constant modulus (CM) property. In [23, 84], Godard and independently Treichler and Agee proposed a method to update equalizer weights based on deviations of the equalizer output from a constant modulus. This popular algorithm is called the constant modulus algorithm (CMA), and it is discussed in section 4.4. This chapter introduces two blind adaptive array methods which both exploit the CM property: (i) the extended CMA (E-CMA) and (ii) the angular CMA (A-CMA). 
The E-CMA is an algorithm capable of simultaneously (i) updating the array directionality and (ii) correcting for Doppler phase offsets. We study the feasibility and computational complexity of the E-CMA in a blind adaptive spatial equalization context in section 4.5. The E-CMA is based on the CMA [84] with an extension by $\mathrm{Xu}$ [94]. Originally, Xu's method was introduced in a spectral equalization context.

Existing blind adaptive array algorithms operate on all transducer outputs and update the entire complex weight vector. These algorithms have no notion of the physical DoA angle of the impinging signal. In contrast, the A-CMA is a blind adaptive algorithm that identifies the presence of mispointing and calculates the required steering angle updates (instead of weight vector updates) to keep track of the desired signal. This approach is attractive in array architectures where distribution of the physical DoA angle is necessary, e.g., mixed-signal hierarchical arrays and collaborative array architectures. An in-depth discussion of the A-CMA is given in section 4.6.

An overview of the most significant results related to blind adaptive array processing, and the E-CMA and A-CMA algorithms can be found in section 4.7.

\subsection{ARRAY THEORY}

In this section, a short overview of array theory is given. The nomenclature and mathematics in this section are mostly based on $[36,43,89]$. A reader experienced in (adaptive) array theory may advance to section 4.4.

\subsubsection{ARRAY TOPOLOGIES}

An array consists of $N$ elements in a linear, planar or volumetric topology. A short discussion on linear and planar topologies is given below. For every topology, $\mathbf{p}_{n}$ is the Cartesian position vector representing the location of the $n^{\text {th }}$ element:

$$
\mathbf{p}_{n}=\left[\begin{array}{c}
p_{\mathrm{x}_{n}} \\
p_{\mathrm{y}_{n}} \\
p_{\mathrm{z}_{n}}
\end{array}\right]
$$

The positions of all array elements is given by the $3 \times N$ matrix $\mathbf{P}$ :

$$
\mathbf{P}=\left[\begin{array}{llll}
\mathbf{p}_{0} & \mathrm{p}_{1} & , \ldots, & \mathbf{p}_{N-1}
\end{array}\right]
$$

\section{Uniform linear array}

In literature, an equidistantly spaced linear array is called a uniform linear array (ULA). An example of a ULA is shown in figure 4.1. Combining signals from the array elements results in a directional dependence. Constructive and destructive interference cause maximum sensitivity for wavefronts arriving perpendicular to the array, since these signals add up coherently. The directions perpendicular and 
parallel to the array are called broadside and endfire, respectively. Section 4.2.5 presents methods to steer an array's directionality. The directionality of a ULA can only be changed in the $x z$-plane. Quantification and visualization of these directionality changes are discussed in section 4.2.7.

In antenna theory, a continuous spatial region that radiates or captures signals is called an aperture [65]. Rather than a single continuous aperture, an array can be considered as a discrete aperture. The linear array, shown in figure 4.1, is an aperture $D$ with a uniform aperture distribution of array elements. The array spacing $d$ and aperture $D$ can be regarded as key parameters in the design of arrays.

\section{Uniform planar array}

A commonly used two-dimensional array topology is the uniform planar array (UPA). This type of array has its elements positioned on a uniform grid. An example of a sixteen-element UPA can be seen in figure 4.2. Just as for a ULA, combining signals from the array elements introduces directionality. By means of appropriate array processing (section 4.2.5), a UPA can steer its directionality into all possible $x y z$-directions.

Note that the array spacing of a UPA in, e.g., the diagonal direction is different from its spacing in the $x$ - and $y$-direction. Consequences of this non-constant array spacing are discussed in section 4.2.7.

\subsubsection{NEAR- AND FAR-FIELD CONDITION}

Arrays have a near-and a far-field region. In the near-field region, large differences in acoustic pressure can be observed for small changes of the observation point. In the far-field region, acoustic pressure differences are more subtle. Pressure disturbances originating from a far-field source can be considered as a plane wave. In an acoustic plane wave, the pressure (at a given time instant) can be considered constant over any plane perpendicular to the propagation direction [50]. A plane wave, originating from a far-field source in the direction $\mathbf{u}$, is shown in figure 4.3.
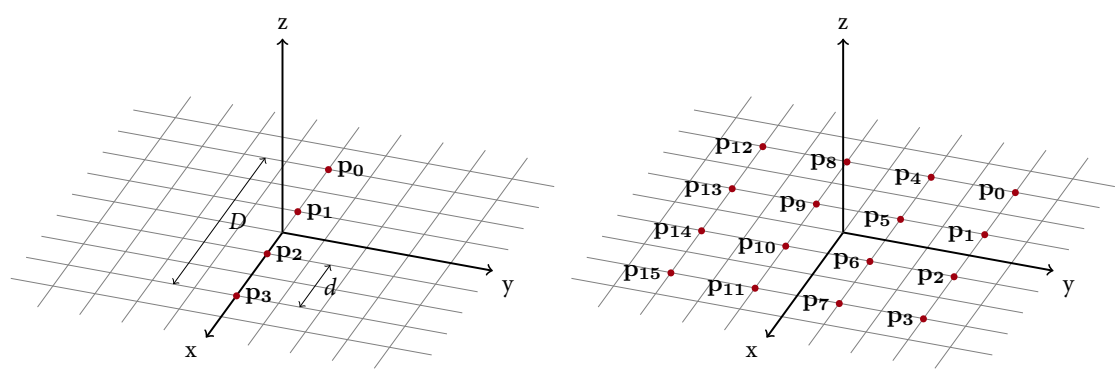

FIGURE 4.1 - Uniform linear array (ULA).

FIgURE 4.2 - Uniform planar array (UPA). 
An acoustic source is considered to be in the far-field if for its radial distance $r$ (in m) holds [68]:

$$
r>\frac{2 D^{2}}{\lambda}
$$

Herein, $D$ is the array aperture (in $\mathrm{m}$ ) and $\lambda$ the wavelength of the highest frequency (in $\mathrm{m}$ ). In this thesis, the source positions are located in the far-field of the array.

\subsubsection{FAR-FIELD SOURCE POSITION}

Source positions relative to the array are generally expressed in a spherical coordinate system. In these coordinates, vectors are described by their polar angle $\theta$, azimuth angle $\phi$ and radius $r$. If the source satisfies the far-field condition (section 4.2.2) then the radius $r$ is superfluous, which means that the unit vector $\mathbf{u}$ $(|\mathbf{u}|=1)$ can be used to represent the angle of incidence of the source signal.

The spherical coordinate system is shown in figure 4.3. Herein, azimuth angle $\phi$ is the angle between the $\mathrm{x}$-axis and $\mathbf{u}$ in the $\mathrm{xy}$-plane. The polar angle represents the angle between the $\mathrm{z}$-axis and $\mathbf{u}$.

The array topology is defined in Cartesian coordinates. It is mathematically convenient to describe the topology and the source position in the same coordinate system. Conversion of the angle of incidence, from spherical to Cartesian coordinates, can be performed by the following coordinate transform:

$$
\mathbf{u}(\theta, \phi)=\left[\begin{array}{l}
u_{x} \\
u_{y} \\
u_{z}
\end{array}\right]=\left[\begin{array}{c}
\sin (\theta) \cos (\phi) \\
\sin (\theta) \sin (\phi) \\
\cos (\theta)
\end{array}\right]
$$

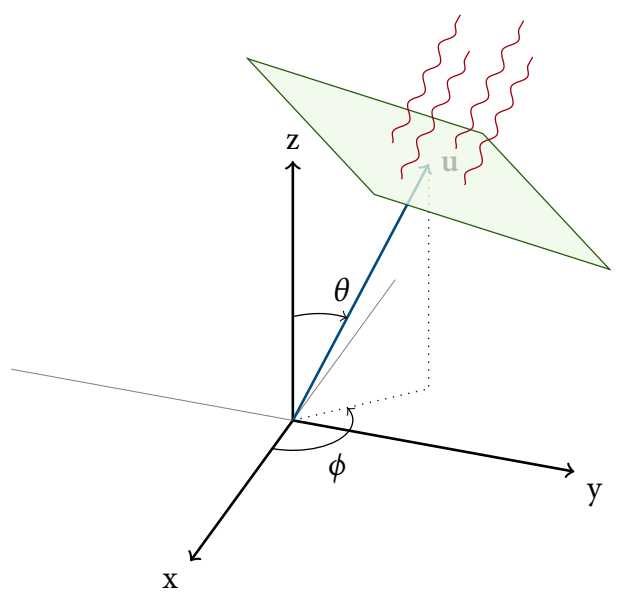

FIgURE 4.3 - Direction vector $\mathbf{u}$ pointing to a far-field source. 
The direction vector $\mathbf{u}$ can be negated to represent the wave propagation direction $\mathbf{a}=-\mathbf{u}$. The wave propagation direction is the unit vector perpendicular to the plane wave pointing in the direction of propagation. The latter is convenient when calculating the array manifold, which is shown in the next section.

\subsubsection{TIME DELAY AND ARRAY MANIFOLD VECTOR}

To quantify the transfer of an impinging wavefront on the array elements, (i) the time delay vector and (ii) the array manifold vector can be calculated. Unless mentioned otherwise, array elements are considered to be isotropic, which means that their individual response is uniform regardless of the signal direction.

The time delay vector $\boldsymbol{\tau}(\theta, \phi)$ contains the propagation times for each array element with respect to the reference point (origin). The vector $\boldsymbol{\tau}(\theta, \phi)$ can be found by calculating the relative path length differences through scalar projection of the positions $\mathbf{p}_{n}$ onto $\mathbf{a}(\theta, \phi)$ and dividing the resulting lengths by the wave propagation speed $c$. Scalar projection (onto a unit vector) can be calculated using the inner product. Therefore, time delay vector $\boldsymbol{\tau}(\theta, \phi)$ can mathematically be written as [89]:

$$
\boldsymbol{\tau}(\theta, \phi)=\left[\begin{array}{c}
\tau_{0}(\theta, \phi) \\
\vdots \\
\tau_{N-1}(\theta, \phi)
\end{array}\right]=\frac{\mathbf{P}^{T} \mathbf{a}(\theta, \phi)}{c} .
$$

For a four-element UPA, calculation of $\boldsymbol{\tau}(\theta, \phi)$ is visualized in figure 4.4. Herein, the wavefront first arrives at element $\mathbf{p}_{0}$, before simultaneously impinging at $\mathbf{p}_{1}, \mathbf{p}_{2}$ and the reference point. Finally, element $\mathbf{p}_{3}$ is affected.

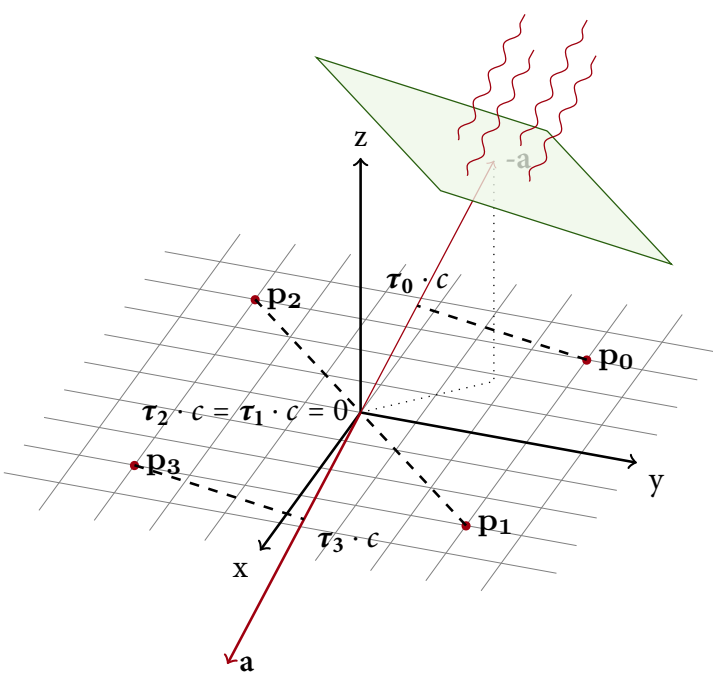

FIgURE 4.4 - Calculation of $\boldsymbol{\tau}(\theta, \phi)$ for a far-field source. 
Instead of having a spatial notion of time, for array processing, it is often more convenient to have a spatial notion of phase. Therefore, the wavenumber $k(\omega)$ is introduced. The wavenumber of a signal is the phase accumulation per unit length and is defined as $\frac{\omega}{c}$. Herein, $\omega$ is the frequency (in $\mathrm{rad} \mathrm{s}^{-1}$ ) and $c$ the wave propagation speed (in $\mathrm{m} \mathrm{s}^{-1}$ ). For example, a signal with wavenumber $k$ has a phase accumulation of $k \cdot l$ after travelling a distance $l$ in the propagation direction.

The relative phase accumulation of a signal, for all elements, can now be written as $k \cdot \mathbf{P}^{T} \mathbf{a}(\theta, \phi)$. These phase accumulations, for a signal with frequency $\omega$, written as phase shifts in the form of complex exponentials, are represented in the array manifold vector $\mathbf{v}(\theta, \phi, \omega)$ [89]:

$$
\mathbf{v}(\theta, \phi, \omega)=e^{-j \cdot k(\omega) \mathbf{P}^{T} \mathbf{a}(\theta, \phi)} .
$$

Herein, the propagation speed $c$ of the wavefront is assumed to be constant.

\subsubsection{ARRAY PROCESSING}

The combining and processing of array signals is called array processing [87] or beamforming [90]. Signal processing structures for array processing are known as beamformers. In this section, various beamformers will be discussed.

The structure of a beamformer highly depends on the bandwidth of the transceived signal with respect to its carrier frequency. The latter relationship is called the fractional bandwidth (FB). The FB was elaborated on in section 3.4.2. Remind that signals are considered narrowband if their FB is one percent or less and that these signals can often be treated as sinusoidal signals.

\section{Array processing methods}

An overview of array processing methods is presented in [36]. A review of these methods is given below. The first method, phase shift based beamforming, can only be used for narrowband signals. The other four methods are suited for both narrowband and wideband signals. Although most of the enumerated methods can be used for both transmit and receive beamforming, in this thesis we will mainly focus on the latter.

i Phase shift based beamforming: Narrowband array signals can be shifted in phase to compensate for the relative phase accumulation experienced by the array elements. The coherent combination of all the shifted signals results in angular regions of directionality known as lobes. The lobe containing the maximum directionality is called the main lobe or main beam. The frequency-dependent phase accumulation, for all elements, is given in eq. 4.6. Wideband signals cannot be treated as sinusoidal signals. Therefore, phase shift based beamforming of wideband signals results in changing locations of the main beam for large changes in the frequency of the impinging signal. The latter phenomenon is called beam squint [47]. 
A common method to implement phase shifts is to make use of complex multiplications. For each complex multiplication within the beamformer, apart from the phase, also the amplitude of each element signal can be set. The latter is called tapering and it can for example be used to broaden the main beam, while simultaneously lowering the directionality for angles outside the main beam [89].

ii Time delay based beamforming: Time delays can be introduced to steer the main beam towards a signal source. The time delays, to account for the path length differences experienced by the array elements, can be found using eq. 4.5 . The combination of the delayed signals is in-phase for every frequency component of the signal and can therefore be used in wideband applications. However, the gain and phase response of a time delay are fixed for all frequencies. Therefore, this beamforming method has limited flexibility.

iii Frequency domain beamforming: A frequency domain beamformer converts the wideband signal received by every distinct array element to the frequency domain using a discrete Fourier transform (DFT). Each bin of the resulting frequency domain representation corresponds to a narrowband part of the original wideband signal [89]. The amplitude and phase of each bin are weighted. Thereafter, corresponding bins are summed and the results are transformed to the time domain by application of the inverse discrete Fourier transform (IDFT). Although calculation of the DFT and IDFT increases computational overhead, this method offers a lot of flexibility.

iv Tapped delay line (TDL) based beamforming: A TDL based beamformer samples the received wideband signal in both time and space by appending multiple delays to each antenna [43]. This structure, which can be regarded as a FIR filter for each antenna, can be seen in figure 4.5. Herein, the output of the $n^{\text {th }}$ array element is denoted by $x_{n}$. The beamformer output is represented by $y$.

TDL based beamforming enables frequency-dependent control of the phase shift and gain for each antenna, without the overhead of conversion to the frequency domain. The beamformer is configured by setting its complex-valued weights. Often, for mathematical convenience, the weights of a TDL based beamformer are concatenated in a $1 \times M N$ row vector:

$$
\begin{aligned}
\mathbf{w}^{H}= & {\left[w^{*}{ }_{0,0}, \ldots, w^{*}{ }_{0, M-1},\right.} \\
& w^{*}{ }_{1,0}, \ldots, w^{*}{ }_{1, M-1}, \\
& \vdots \\
& \left.w^{*}{ }_{N-1,0}, \ldots, w_{N-1, M-1}^{*}\right] .
\end{aligned}
$$

Much literature is available on this flexible beamforming method [36, 43, 90].

$\mathrm{v}$ Beamspace beamforming: Ordinary beamformers combine the pre-processed outputs of multiple array elements. In contrast, a beamspace beamformer combines outputs of multiple beamformers. Therefore, such a beamformer is said to operate in beamspace instead of element space [55]. A common technique 


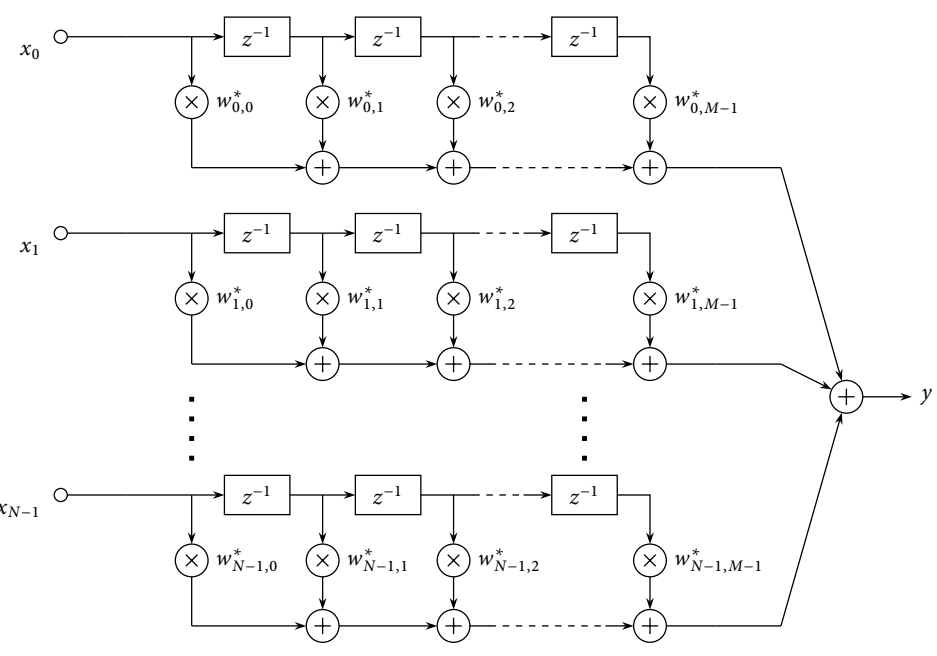

FIGURE 4.5 - TDL based beamformer

to convert array inputs to beamspace is the DFT. The $N$ array input signals are directly fed into an $N$-point DFT. The output of this 'spatial' DFT represents beams in $N$ different directions. A linear combination of samples in beamspace can be used to generate beams in other directions. Beamspace processing can be applied for both narrowband and wideband signals [91].

\subsubsection{ARRAY RESPONSE VECTOR AND BEAMFORMER RESPONSE}

The transfer of an impinging signal to the array elements, in terms of relative phase accumulations, is described by the array manifold vector (eq. 4.6). To find a mathematical transfer function for the entire beamformer, from the impinging signal to the output, it is convenient to also define the array response vector. The array response vector is the transfer of a source signal, through the beamformer structure, to the point at which the weights are applied. By appropriately defining the weight vector, the entire beamformer response can be expressed as the inner product of the weights and the array response vector. To illustrate this concept, this section elaborates on calculation of the beamformer response for the TDL based beamformer (figure 4.5) and the phase shift based beamformer.

\section{TDL based beamformer response}

The transfer through a TDL based beamformer structure with $M$ delay taps, to the point at which the weights are applied, is expressed by array response vector $\mathbf{d}(\theta, \phi, \omega)$ :

$$
\mathbf{d}(\theta, \phi, \omega)=\mathbf{v}(\theta, \phi, \omega) \otimes e^{-j \omega \mathbf{m} T_{s}}, \quad \mathbf{m}=[0,1, \ldots, M-1]^{T} .
$$


Herein, $\omega$ denotes frequency, $\theta$ and $\phi$ the incidence angle, $\otimes$ the Kronecker product and $T_{s}$ the sample period (in s). The $N M \times 1$-element column vector $\mathbf{d}(\theta, \phi, \omega)$ can be regarded as the phase accumulations caused by spatio-temporal sampling of the impinging signal [90].

For the TDL based beamformer, to find the beamformer response $B(\theta, \phi, \omega)$ of the entire structure, the weight vector (eq. 4.7) and the array response vector are combined by calculating their inner product:

$$
B(\theta, \phi, \omega)=\mathbf{w}^{H} \mathbf{d}(\theta, \phi, \omega) .
$$

Notice that $B(\theta, \phi, \omega)$ is a frequency domain description of the beamformer response. The beamformer output $y$ for an impinging signal $s$ can be calculated in either the frequency domain or the time domain. For analysis and to compare beamformer responses it is often convenient to use frequency domain descriptions. In this chapter, we are particularly interested in the transient behaviour of (adaptive) beamformers. Therefore, we prefer to work with time domain descriptions of the source signal and the beamformer output.

To calculate the discrete-time beamformer output $y[k]$ for the structure in figure 4.5, first the effect of spatial sampling is modelled by defining a vector $\mathbf{x}(t)$ with $N$ delayed versions of the continuous-time source signal $s(t)$ according to the time delay vector $\boldsymbol{\tau}(\theta, \phi)$ (eq. 4.5):

$$
\mathbf{x}(t)=\left[\begin{array}{c}
x_{0}(t) \\
x_{1}(t) \\
\vdots \\
x_{N-1}(t)
\end{array}\right]=\left[\begin{array}{c}
s\left(t-\tau_{0}\right) \\
s\left(t-\tau_{1}\right) \\
\vdots \\
s\left(t-\tau_{N-1}\right)
\end{array}\right] .
$$

The $n^{\text {th }}$ element of $\mathbf{x}(t)$ is the continuous-time signal received by the $n^{\text {th }}$ array element. Notice that, for mathematical clarity, the dependence on the incidence angle is dropped.

In the remainder of this thesis, we assume that each array element is a quadrature receiver equipped with an ADC. The discrete-time complex baseband signals generated by these $N$ array elements are represented by $x_{0}[k] \ldots x_{N-1}[k]$. Herein, $k$ is an index for the sampling instants. The discrete-time beamformer output $y[k]$ for the TDL based beamformer in figure 4.5 can now be expressed as the sum of $N$ convolutions [90]:

$$
y[k]=\sum_{n=0}^{N-1} \sum_{m=0}^{M-1} w_{n, m}^{*} x_{n}[k-m] .
$$

\section{Phase shift based beamformer response}

The structure of a phase shift based beamformer is equal to a TDL based beamformer without delay lines. Therefore, a phase shift based beamformer has an 
array response vector that is similar to its array manifold vector: $\mathbf{d}(\theta, \phi, \omega)=$ $\mathbf{v}(\theta, \phi, \omega) \otimes e^{-j \omega 0 T_{s}}=\mathbf{v}(\theta, \phi, \omega) \otimes 1=\mathbf{v}(\theta, \phi, \omega)$.

The response $B(\theta, \phi, \omega)$ of a phase shift based beamformer is equal to eq. 4.9. However, often the independent variable $\omega$ is dropped, since the impinging signal can be treated as a sinusoidal signal with a constant frequency $\left(\omega=2 \pi \cdot f_{h}\right)$ :

$$
B(\theta, \phi)=\mathbf{w}^{H} \mathbf{d}(\theta, \phi) .
$$

If the discrete-time complex baseband signals generated by the array elements are represented in an $N$-element column vector $\left(\mathbf{x}[k]=\left[x_{0}[k], \ldots, x_{N-1}[k]\right]^{T}\right)$ then the discrete-time beamformer output can be written as:

$$
y[k]=\mathbf{w}^{H} \mathbf{x}[k] .
$$

Usually, the weights are normalized to ensure a unit magnitude response in the direction of maximum sensitivity. If the weights are chosen to exactly compensate for the array response vector then the beamformer is known as a conventional beamformer [83].

\subsubsection{PERFORMANCE ANALYSIS}

\section{Array pattern}

To evaluate the performance of a beamformer, its response can be plotted for a range of incidence angles (and frequencies). This response is known as the array pattern or the beam pattern. Generally, when plotting these patterns, the array steering angle is set to the broadside direction. The array pattern of a conventional narrowband beamformer ${ }^{1}$ with a four-element ULA topology is shown in figure 4.6. Herein, the main beam (or main lobe) and two lobes with a smaller intensity can be recognized. The smaller lobes are known as sidelobes and the angles corresponding to zero intensity, due to destructive interference, are called nulls. The topology, which gives rise to figure 4.6 , has a spacing between the elements equal to half the wavelength of the signal of interest $\left(d=\frac{1}{2} \lambda\right)$. Such a spacing is often referred to as ideally spaced. To study the effect of altering key parameters of the array, it is helpful to realize there exists a discrete Fourier relation between the discrete aperture distribution and the far-field array pattern [8]. For example, choosing an array spacing larger than half the wavelength of the signal of interest can be considered an undersampled aperture distribution. The DFT of an undersampled time signal has spectral aliases. Analogously, the DFT of an undersampled aperture has spatial aliases.

\footnotetext{
${ }^{1} \mathrm{~A}$ (normalized) conventional narrowband beamformer steered into the broadside direction has $N$ weights which are all set to $1 / N$.
} 


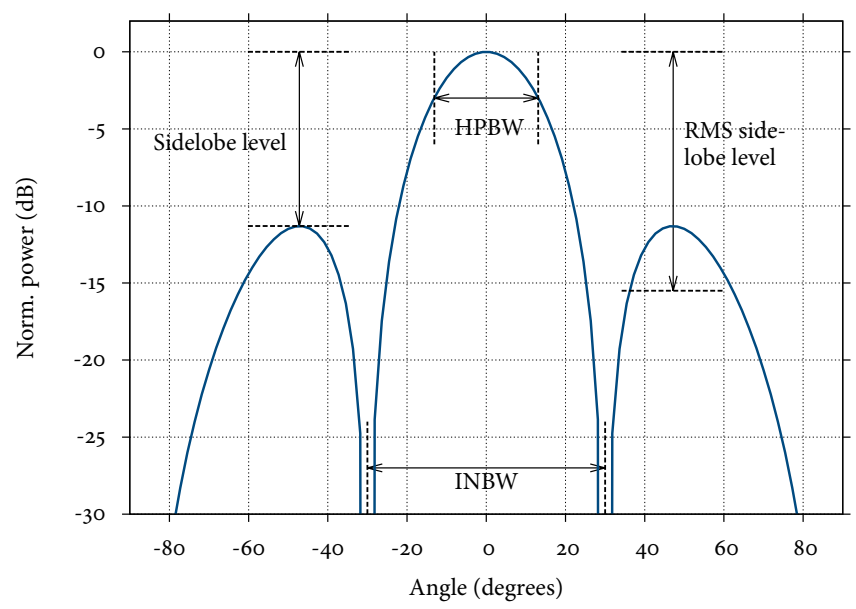

FIGURE 4.6 - Array pattern of a four-element ULA $\left(d=\frac{1}{2} \lambda\right)$.
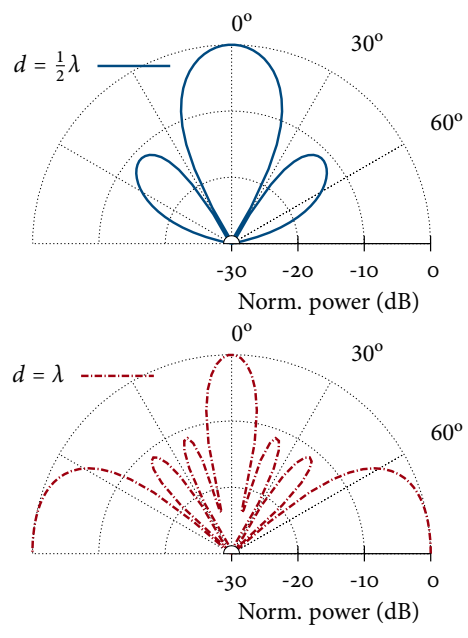

FIGURE 4.7 - Array patterns in polar form of a four-element ULA with different spacings.

Spatial aliases, better known as grating lobes, were shortly discussed in chapter 3 . To illustrate grating lobes, the array patterns of a four-element ULA with a halfwavelength and a wavelength spacing $\left(d=\frac{1}{2} \lambda\right.$ and $\left.d=\lambda\right)$ are shown in figure 4.7. Herein, both patterns are illustrated in a spherical coordinate system (polar plot). Such a representation gives more insight in the actual directionality of an array. In the array pattern with $d=\lambda$, grating lobes can be recognized in the positive and negative endfire directions. 


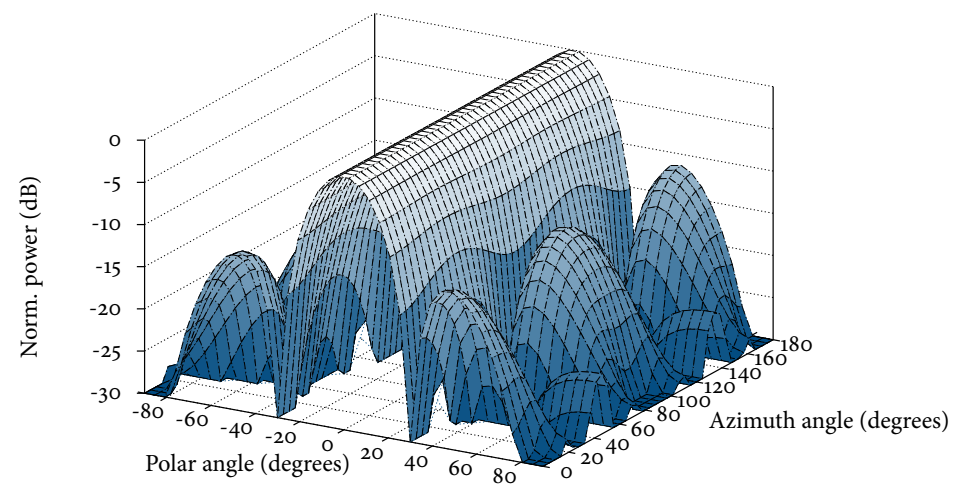

FIGURE 4.8 - Array pattern of a sixteen-element UPA $\left(d=\frac{1}{2} \lambda\right)$.

The array pattern of a $4 \times 4$ UPA is illustrated in figure 4.8 . The array response of a UPA depends on both the polar and the azimuth incidence angle. Most planar arrays are attached to a ground plane to eliminate lobes on the opposite site of the plane [48]. For incidence signals parallel to the diagonal axis, elements on both the $x$ - and the $y$-axis contribute to the directionality. Therefore, the sidelobe reduction is larger for diagonally impinging signals than for incidence signals parallel to either one of the principal axes.

\section{Quantitative analysis}

For quantitative evaluation of array patterns, performance measures are needed. A short overview of commonly used performance measures is presented below. As an example, the enumerated measures are annotated in figure 4.6.

i Half-power beamwidth: The width of the main beam is often specified in terms of the angular span between the $-3 \mathrm{~dB}$ points. This distance is known as the half-power beamwidth (HPBW) or the $3 \mathrm{~dB}$ beamwidth [3].

ii Inter-null beamwidth: The inter-null beamwidth (INBW) of the main beam is the angular span between the nulls surrounding the main beam [3].

iii (Root mean square) sidelobe level: The sidelobe level is specified as the intensity difference between the peak of the main beam and the highest sidelobe. Often, the root mean square (RMS) average of the entire sidelobe is a more important measure [74]. In figure 4.6 this RMS sidelobe level is $-15.5 \mathrm{~dB}$.

Other important measures are the directivity and the array gain [89]. Nevertheless, since we mainly elaborate on measures directly related to blind equalization, directivity and array gain are not discussed. 
In dynamic environments, the weights of an array need to be adjusted to compensate for the changing signal (conditions and) directions. An adaptive array algorithm is required to calculate appropriate weight adjustments. The general structure for adaptive array processing can be seen in figure 4.9. Herein, $\mathbf{x}, \mathbf{w}$ and $y$ are, respectively, the baseband signals from the array elements, the weight vector and the beamformer output.

The beamformer output $\hat{s}$ is an estimate of the baseband source signal $s$. Ideally, the beamformer output $y$ is equal to this source signal. Nevertheless, the beamformer is allowed to impose a delay and complex gain on the source transmission. Chapter 5 further elaborates on ideal equalization.

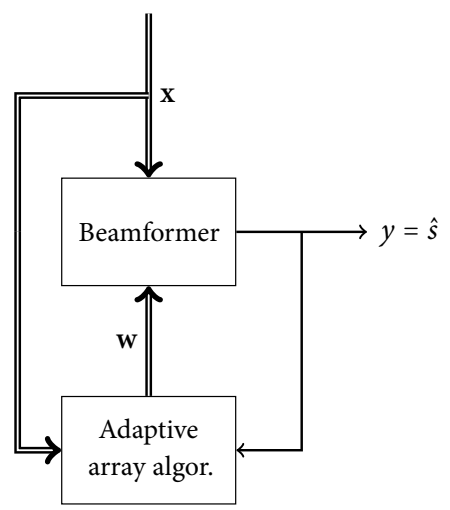

FIGURE 4.9 - General form of an adaptive beamformer.

Adaptive array algorithms can be categorized in three classes [3]:

i Temporal reference: Temporal reference algorithms use a known training sequence $r$ embedded in the source signal by the transmitter, to determine adjustments for the array weight vector $\mathbf{w}$. The optimum weight vector for reception of a single source out of noise plus interferers is the Wiener-Hopf optimum $\mathbf{w}_{\text {opt }}$ [3] [87]. Most temporal reference algorithms asymptotically convergence to this solution. Weight vector $\mathbf{w}_{\mathrm{opt}}$ can be found by calculating $\mathbf{w}_{\mathrm{opt}}=\mathrm{R}_{\mathrm{xx}}^{-1} \boldsymbol{\gamma}_{\mathrm{rx}}$. Herein, $\boldsymbol{R}_{\mathrm{xx}}$ is the spatial autocovariance matrix, which describes the degree of correlation between samples from different array elements for the same time instant $\left(\boldsymbol{R}_{\mathbf{x x}}=E\left[\mathbf{x x}^{H}\right]\right)$. And, $\gamma_{r \mathbf{x}}$ is the cross-covariance vector, which describes the correlation between samples from different array elements and the deterministic training sequence $r[k]\left(\gamma_{r \mathbf{x}}=E\left[r \mathbf{x}^{*}\right]\right)$.

Temporal reference algorithms can only be used in case training sequences are available. The lengthening of transmission time slots, due to training data, increases the energy consumption of communicating nodes. A real world radio 
frequency (RF) communication system that utilizes training data is the global system for mobile communication (GSM). Herein, the training data accounts for $\mathbf{2 2} \%$ communication overhead [52]. We aim for a solution with little or no overhead due to training data.

ii Spatial reference: Spatial reference algorithms exploit the correlation characteristics amongst samples from different array elements measured at the same time instant. An important application of these algorithms is (high accuracy) DoA angle estimation of all impinging signals. Following upon this DoA angle estimation, a pattern can be synthesized that points the main beam in the direction of the signal of interest, while simultaneously nulling (possible) interfering signals.

Some methods in this class are: multiple signal classification (MUSIC), estimation of signal parameters by rotational invariance techniques (ESPRIT) and maximum likelihood (ML) based techniques [70] [59] [98]. Typically, the better the accuracy of a spatial reference algorithm, the higher its computational complexity [3].

In general, if the array manifold has imperfections and is therefore not accurately known, the performance of spatial reference algorithms will degrade. The classes of temporal reference and blind adaptive algorithms are less sensitive to array imperfections.

iii Blind adaptive (array) algorithms: Blind adaptive algorithms do not require training data. A key challenge in the development of these algorithms is to find a substitute for this absent reference. In general, blind algorithms update the equalizer weights based on structural and statistical properties of the equalizer output and the received signals. In this chapter, blind adaptive algorithms are discussed and applied in a spatial equalization context. Chapter 5 elaborates on blind equalization in a spectral context.

Blind adaptive array processing can be based on second-order statistics (SOS) or on higher-order statistics (HOS). In general, HOS-based methods are more elaborate and computationally intensive, but their performance to focus on (non-Gaussian) source signals is improved with respect to SOS-based methods [52].

The class of blind adaptive array processing algorithms that exploits HOS can be subdivided into explicit HOS-based and implicit HOS-based methods. Explicit HOS-based methods directly operate on higher-order statistical properties of the input signals, such as the skewness or the kurtosis, to calculate weight adjustments. The relation between HOS and ideal equalization is discussed in more detail in chapter 5 and appendix A.

Implicit HOS-based methods optimize a nonlinear cost function. The implicit dependence on HOS can be made explicit by expanding a cost function's nonlinearity in terms of its higher-order moments. For the cost function employed in the remainder of this chapter, this expansion can be found in [96]. 
As explained in section 4.1, for power-efficiency, we employ QPSK modulation. QPSK is a form of PSK modulation that uses four different phases of the carrier wave to represent the data symbols to be transmitted. Since exclusively the phase of the carrier wave is adjusted, the output of a PSK modulator always has a constant modulus. In this section, we elaborate on exploiting the constant modulus (CM) property of a source signal to serve as a reference signal in blind array processing.

\subsubsection{HISTORY}

The CMA uses a nonlinear cost function $J_{\mathrm{CMA}}$ to penalize the deviation of an equalizer output from a constant modulus. Historically, the concept of applying a memoryless nonlinear cost function to the output of an equalizer to act as a substitute for a reference signal was invented by Sato [69]. Specifically exploiting the CM property of the source signal to generate such a reference was proposed by Godard [23]. Originally, Godard's method was developed to perform fast startup equalization in multipoint networks. Independently of Godard, a special case of this idea was published by Treichler and Agee [84]. Treichler and Agee named their algorithm the constant modulus algorithm (CMA).

\subsubsection{CMA COST FUNCTION}

In the context of array processing, the CMA cost function $J_{\mathrm{CMA}}$ is defined as the expected deviation of the squared modulus beamformer output $y$ with respect to the constant $R_{2}$ [26]:

$$
J_{\mathrm{CMA}}=E\left\{\left(|y[k]|^{2}-R_{2}\right)^{2}\right\} .
$$

Herein, $E$ is the expected value and $k$ an index for the sampling instants. Since CMA employs symbol-rate spaced equalization, ideally, the sampling instants coincide with the (optimally timed) symbol instants. Therefore, $k$ can also be thought of as a symbol index.

$R_{2}$ is known as the Godard parameter and is chosen such that the gradient of $J_{\text {CMA }}$ is zero when minimum costs are reached and is written as [23]:

$$
R_{2}=\frac{E\left[|s[k]|^{4}\right]}{E\left[|s[k]|^{2}\right]} .
$$

Herein, $s[k]$ represents the $k$-th symbol transmitted by the source. For an ideal QPSK transmission, the constant $R_{2}$ is equal to one. Since we only consider QPSK transmissions, in the remainder of this chapter, the constant $R_{2}$ is substituted by one.

The CMA cost function $J_{\mathrm{CMA}}$ is illustrated in figure 4.10. The $\mathrm{x}$-axis shows the real part of the equalizer output $y$, the $y$-axis shows the imaginary part of $y$ and the $\mathrm{z}$-axis shows the corresponding costs $J_{\mathrm{CMA}}$. Costs are completely minimized if 


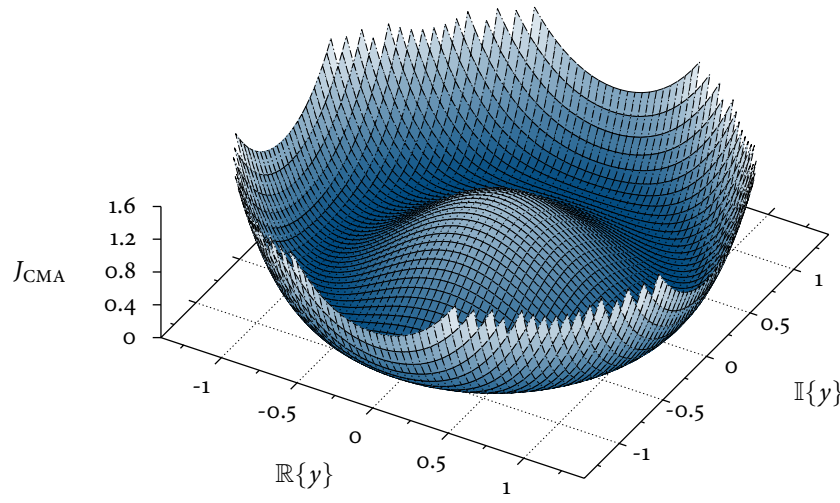

FIGURE 4.10 - Surface plot of the $J_{\mathrm{CMA}}$ cost function $\left(R_{2}=1\right)$

symbols at the output of the beamformer have unit modulus. Clearly, the latter is independent of the phase offset that these symbols might experience.

\subsubsection{CMA COST MINIMIZER}

The output of a narrowband beamformer $y$ can be expressed as $\mathbf{w}^{H} \mathbf{x}$ (eq. 4.13). The aim of the CMA is to minimize $J_{\mathrm{CMA}}$ by adjusting the weight vector $\mathbf{w}$. As in [23], we employ a stochastic gradient descent technique to attain this goal. In literature, other optimization techniques are considered for cost minimization [30]. It is worth mentioning that an analytical solution to the CM criterion, for a finite set of samples and antennas, is derived by van der Veen and Paulraj [88]. An extensive overview of this analytical solution and other optimization approaches is not within the scope of this thesis.

The CMA cost function is of type $\mathbb{C} \rightarrow \mathbb{R}$. Since $J_{\text {CMA }}$ is not complex differentiable, Wirtinger calculus is employed to find the maximum rate of change of $J_{\text {CMA }}$ with respect to $\mathbf{w}$. From the overview of Wirtinger calculus in [9], we learn that the maximum slope of a real scalar function of a complex vector, such as $J_{\mathrm{CMA}}(\mathbf{w})$, can be found by its gradient with respect to $\mathbf{w}^{*}$ (treated independently of $\mathbf{w}$ ). Herein, $\mathbf{w}^{*}$ is the complex conjugate of $\mathbf{w}$. A more mathematically rigor discussion of Wirtinger calculus is given in section 5.4.1.

By defining the gradient operator as $\nabla=\nabla_{\mathbf{w}^{*}}$ (Wirtinger calculus), the maximum rate of change of $J_{\mathrm{CMA}}$ with respect to $\mathbf{w}$ can be found as follows:

$$
J_{\mathrm{CMA}}=E\left\{\left(|y|^{2}-1\right)^{2}\right\}
$$




$$
\begin{aligned}
& \nabla J_{\mathrm{CMA}}=2 \cdot E\left\{\left(|y|^{2}-1\right) \cdot \nabla\left(\left|\mathbf{w}^{H} \mathbf{x}\right|^{2}-1\right)\right\} \\
& \nabla J_{\mathrm{CMA}}=2 \cdot E\left\{\left(|y|^{2}-1\right) \cdot \nabla\left(\mathbf{w}^{H} \mathbf{x} \mathbf{x}^{H} \mathbf{w}-1\right)\right\} .
\end{aligned}
$$

Continue using the fact that the values of $\mathbf{x} \mathbf{x}^{H} \in \mathbb{C}^{N \times N}$ are constant with respect to $\mathbf{w}$, such that $\nabla \mathbf{w}^{H} \mathbf{x} \mathbf{x}^{H} \mathbf{w}=\mathbf{x} \mathbf{x}^{H} \mathbf{w}$ holds:

$$
\begin{aligned}
& \nabla J_{\mathrm{CMA}}=2 \cdot E\left\{\left(|y|^{2}-1\right) \cdot \mathbf{x} \mathbf{x}^{H} \mathbf{w}\right\} \\
& \nabla J_{\mathrm{CMA}}=2 \cdot E\left\{\left(|y|^{2}-1\right) \cdot y^{*} \mathbf{x}\right\} .
\end{aligned}
$$

The weight vector $\mathbf{w}$ is updated in the direction of the negative gradient to minimize $J_{\text {CMA }}$. Mathematically, this can be written as [84]:

$$
\mathbf{w}[k+1]=\mathbf{w}[k]-\mu \nabla_{\mathbf{w}} J_{\mathrm{CMA}} .
$$

The factor $\mu$ determines the convergence rate of the gradient descent. Based on eq. 4.17 the cost minimizer for the stochastic gradient descent version of the CMA can be given as:

$$
\mathbf{w}[k+1]=\mathbf{w}[k]-\mu \cdot\left(|y[k]|^{2}-1\right) \cdot y[k]^{*} \mathbf{x}[k] .
$$

Herein, $\mu$ absorbs the factor 2 . Choosing a convergence factor is discussed in section 4.4.5. The gradient descent version of the CMA is often referred to as the stochastic gradient CMA (SG-CMA) [97].

\section{Choosing an initial weight vector}

The CMA cost function $J_{\mathrm{CMA}}$ is a nonconvex function of the equalizer weights [26]. Therefore, depending on the initial weight vector, the minimizer (eq. 4.19) may converge to a local minimum [26]. The latter is often referred to as ill-convergence.

Choosing an initial weight vector that guarantees global convergence is still an open problem [26]. Li and Ding conjecture that the CMA will converge to the desired optimum if a (sufficiently long) weight vector initialized with a nonzero center tap is used [41]. In our spatial equalization experiments, the array weight vector is initialized to steer the main beam in the broadside direction.

\subsubsection{COMPUTATIONAL COMPLEXITY}

An analysis on the computational complexity of the CMA is given to gather insight on the scalability of the algorithm for arrays with a large number of elements. A block diagram of the elementary operations required for an update of the weight vector can be seen in figure 4.11. In this block diagram, single-line arrows indicate real values, double-line arrows indicate complex values and thick double-line arrows indicate vectors of complex values. 


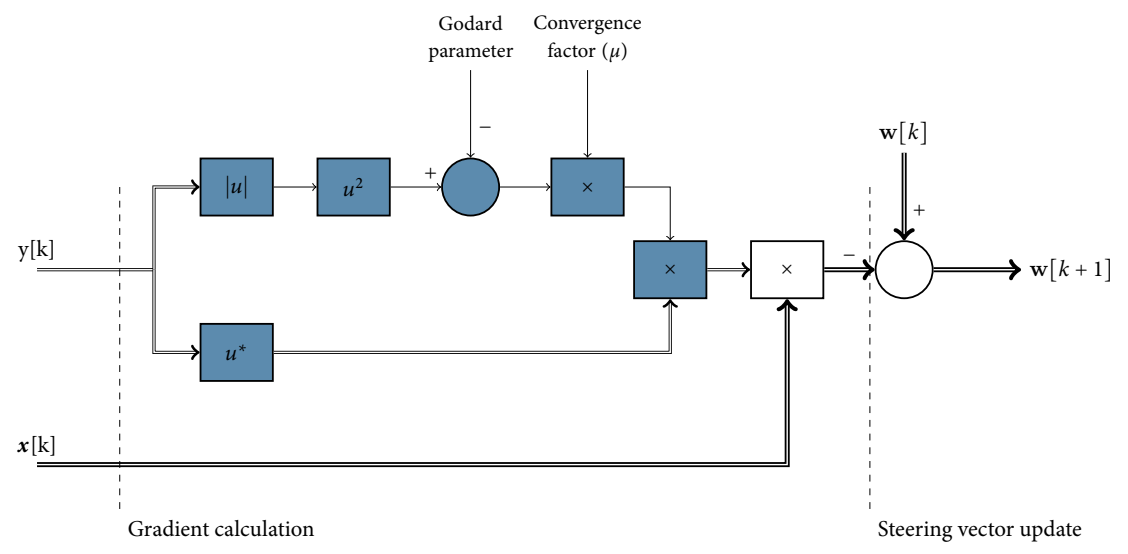

The computational complexity of the CMA can be determined by counting the number of complex multiply-accumulate (MAC) operations. Since the number of operations executed by the highlighted blocks (figure 4.11) does not dependent on the number of array elements, the complexity of the CMA grows linearly with the number of array elements.

\subsubsection{EMPIRICAL PERFORMANCE}

This section elaborates on the performance of the conventional CMA for empirical multi-channel data captured during the dive-center experiment (section 3.6.2). Although the CMA is a prominent technique in many existing RF applications [30], its practical employment in underwater acoustic communication is less prevalent.

\section{Wiener weight vector}

If the reference signal is known, the optimum array weights $\mathbf{w}_{\text {opt }}$ (in the MSE sense) can be calculated using the Wiener-Hopf equation (section 4.3). Based on our empirical data set, the scatter plot and the array pattern for beamforming with optimum weights are shown in figure 4.12. Additionally, the pattern for conventional beamforming in the direction of the strongest signal power is illustrated. Apparently, compared to conventional beamforming, slightly shifting the main beam and the two leftmost nulls to the right improves the MSE of the beamformer output.

\section{CMA learning curve}

The performance of the gradient descent version of the CMA can be studied by looking at the learning curve of its cost function $J_{\mathrm{CMA}}$. A key factor for stochastic gradient descent methods is the convergence factor $\mu$, since it determines the convergence speed and the stability during optimization. The choice for a particular 

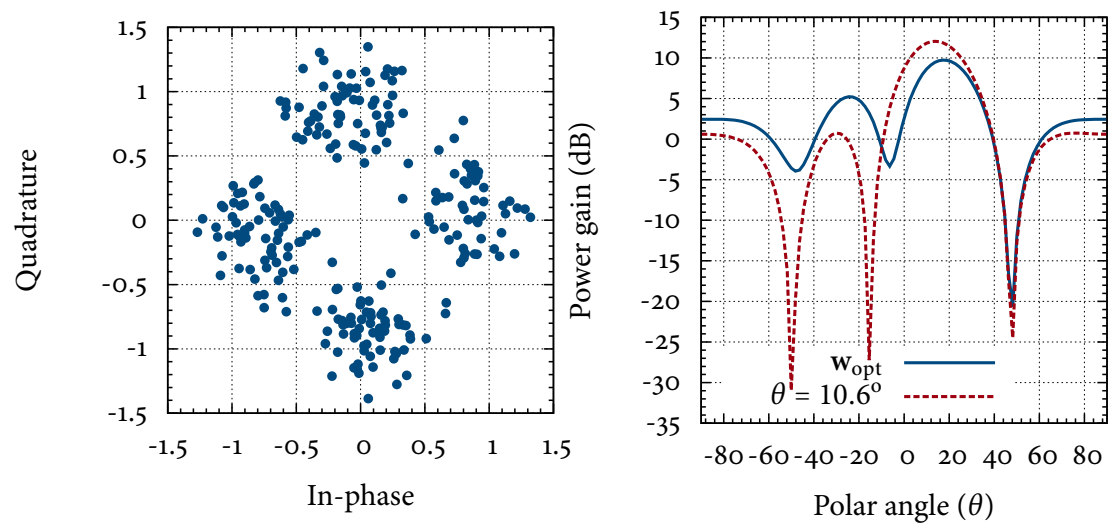

FIGURE 4.12 - left: Scatter plot for $\mathbf{w}_{\text {opt }}$. right: Pattern for conventional weights and $\mathbf{w}_{\text {opt }}$.

convergence factor (also) depends on the amplitude of the input signals. To eliminate this dependence, e.g., the normalized CMA (NCMA) can be employed [31].

For blind equalization of our normalized ${ }^{2}$ multi-channel recordings, we experimented with large convergence factors $(\mu=.25$ and $\mu=.5)$. The learning curve of the CMA applied to these recordings can be seen in figure 4.13. Since we are only interested in revealing the trend, the results are smoothed by a moving average filter. For comparison, also the CMA costs corresponding to the optimum weights $\mathbf{w}_{\text {opt }}$ are shown. In our experiment, even for large convergence factors, the CMA descends asymptotically towards the optimum solution.

\section{MSE performance}

Another common method to evaluate an equalizer is to determine the MSE of its output signal. This error is known as the predetection error. The CMA does not correct for phase offsets in the beamformer output signal. The uncorrected phase should not affect the MSE level of its output signal, therefore the following method is used to calculate the MSE:

$$
\mathrm{MSE} \triangleq \min _{\alpha} E\left\{\left|\mathbf{w}[k]^{H} \mathbf{x}[k]-e^{j \alpha} s[k]\right|^{2}\right\}
$$

Herein, $s[k]$ represents the (noiseless) transmitted symbol $k$. This unconventional method of MSE calculation was introduced by Treichler and Agee in [84].

${ }^{2}$ The signals of the array elements are normalized with respect to their maximum absolute values. 
Estimates for the MSE levels for Wiener beamforming and the CMA can be seen in figure 4.14. As expected, the almost exponential convergence of the learning curves in figure 4.13 results in a linearly decreasing MSE (on a dB scale) in figure 4.14.

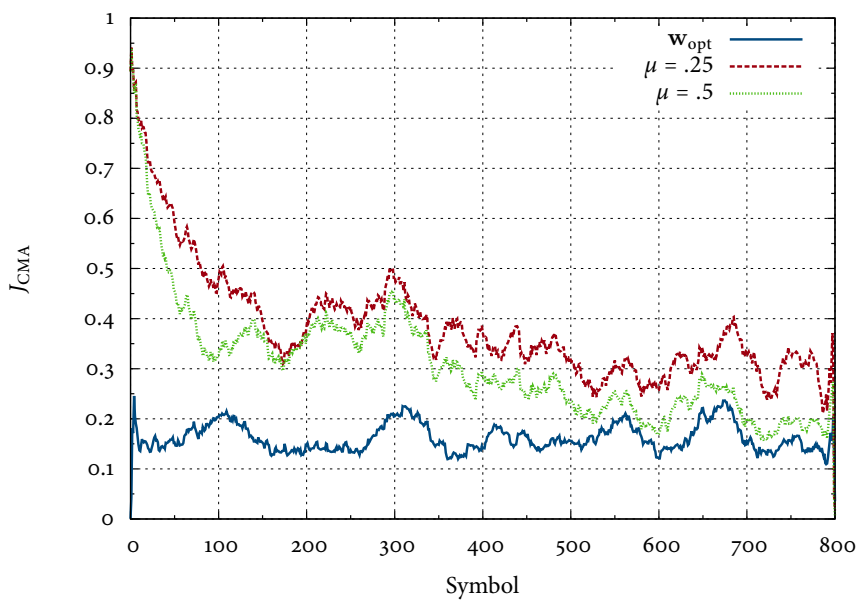

FIGURE 4.13 - Blind equalization of empirical data using the CMA.

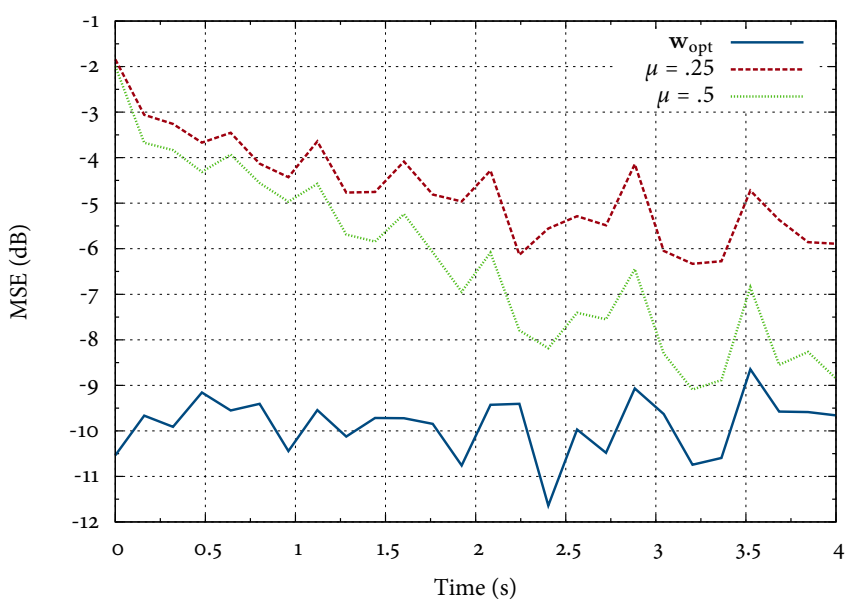

FIGURE 4.14 - MSE levels during application of the CMA. 
In addition to UW-ASNs, there is also a growing interest in application of autonomous underwater vehicles (AUVs) for underwater monitoring. Extending these AUVs to communicate acoustically requires hull-mounted transducers. An example of an AUV with a four-element hull-mounted array is given in [21].

Movement of a hull-mounted array introduces modulus and phase deviations in the QPSK modulated output of a beamformer due to mispointing and the Doppler effect, respectively. The CMA does not correct for phase rotations of the beamformer output. Traditionally, phase offsets in the beamformer output, caused by the Doppler effect, are corrected in the derotator of the QPSK demodulator [95]. Such a derotator is often implemented by a digital delay locked loop (DDLL) or a phase locked loop (PLL), which results in a more complicated structure, longer convergence times and a larger signal degradation [54].

So far no studies have been performed on the feasibility and computational complexity of the CMA with the extended cost function of Xu [94] for blind beamforming of underwater PSK transmissions. The latter approach is accounted for in this section and enables direct correction of phase offsets in the beamformer output. Alternative methods, such as techniques based on the finite-alphabet property [81], are not being discussed in this thesis.

\subsubsection{E-CMA COST FUNCTION}

To compensate for both phase and modulus deviations in a (QPSK modulated) beamformer output, the CMA cost function needs to be altered. As explained in section 4.4.2, the conventional CMA optimizes the weights based on (only) the expected deviation of the squared modulus of the beamformer output with respect to a constant value. For a normalized QPSK modulated beamformer output this constant value is one, since the QPSK symbols lie on the unit circle.

The CMA for QPSK based receivers can be improved by adjusting the weights based on both modulus and phase deviations. The equal phase distribution of a QPSK modulated signal $y$ can mathematically be expressed by $\sin \left(2 y_{<}\right)=0$, where $y_{<}$ represents the instantaneous phase angle of $y$. This phase distribution was used by $\mathrm{Xu}$ [94], originally in a spectral equalization context, to derive a new cost function $J_{\mathrm{E}-\mathrm{CMA}}$ based on both modulus and phase deviations:

$$
J_{\mathrm{E}-\mathrm{CMA}}=E\left\{\left(|y|^{2}-1\right)^{2}\right\}+E\left\{\sin ^{2}\left(2 y_{<}\right)\right\} .
$$

The cost function $J_{\mathrm{E}-\mathrm{CMA}}$ is illustrated in figure 4.15. The $\mathrm{x}$-axis shows the real part of the equalizer output $y$, the $y$-axis the shows the imaginary part of $y$ and the $z$-axis shows the corresponding costs $J_{\mathrm{E}-\mathrm{CMA}}$. Minimum costs are reached whenever $y$ simultaneously has a unit modulus and a phase equal to one of the QPSK symbol phases. 


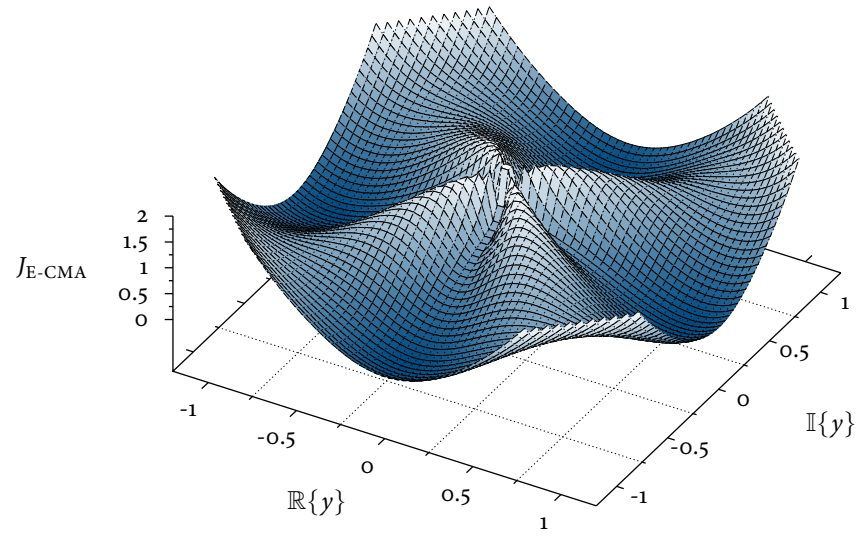

FIGURE 4.15 - Surface plot of the $J_{\mathrm{E}-\mathrm{CMA}}$ cost function.

Rewriting the instantaneous phase angle of $y$ in terms of $\mathbf{w}$ and $\mathbf{x}$ results in [94]:

$$
\begin{aligned}
y_{<} & =\arctan \left(\frac{y-y^{*}}{j\left(y+y^{*}\right)}\right) \\
& =\arctan \left(\frac{\mathbf{w}^{H} \mathbf{x}-\mathbf{x}^{H} \mathbf{w}}{j\left(\mathbf{w}^{H} \mathbf{x}+\mathbf{x}^{H} \mathbf{w}\right)}\right) .
\end{aligned}
$$

Thereafter, $J_{\mathrm{E}-\mathrm{CMA}}$ can be rewritten in terms of $\mathbf{w}$ and $\mathbf{x}$ :

$$
\begin{aligned}
J_{\mathrm{E}-\mathrm{CMA}}(\mathbf{w})= & E\left\{\left(\left|\mathbf{w}^{H} \mathbf{x}\right|^{2}-1\right)^{2}\right\}+ \\
& E\left\{\sin ^{2}\left(2 \cdot \arctan \left(\frac{\mathbf{w}^{H} \mathbf{x}-\mathbf{x}^{H} \mathbf{w}}{j\left(\mathbf{w}^{H} \mathbf{x}+\mathbf{x}^{H} \mathbf{w}\right)}\right)\right)\right\} .
\end{aligned}
$$

\subsubsection{E-CMA COST MINIMIZER}

Similar to the conventional version of the CMA, the cost function $J_{\mathrm{E}-\mathrm{CMA}}$ can be iteratively minimized using a stochastic gradient descent. The cost minimizer of the E-CMA, based on the gradient $\nabla_{\mathbf{w}} J_{\mathrm{E}-\mathrm{CMA}}$, can be written as [94]:

$$
\begin{aligned}
\mathbf{w}[k+1] & =\mathbf{w}[k]-\mu \cdot \nabla_{\mathbf{w}} J_{\mathrm{E}-\mathrm{CMA}} \\
& =\mathbf{w}[k]-\mu \cdot \frac{2 j\left(|y[k]|^{4}-|y[k]|^{2}\right)+\sin \left(4 y_{<}[k]\right)}{j \cdot y[k]} \cdot \mathbf{x}[k] .
\end{aligned}
$$


To determine the scalability of the E-CMA, a short analysis of its computational complexity is given. The basic operations of the cost minimizer are shown in figure 4.16. Herein, the number of operations executed by the highlighted blocks does not depend on the number of array elements. Therefore, similar to the CMA, the complexity of the E-CMA grows linearly with the number of array elements.

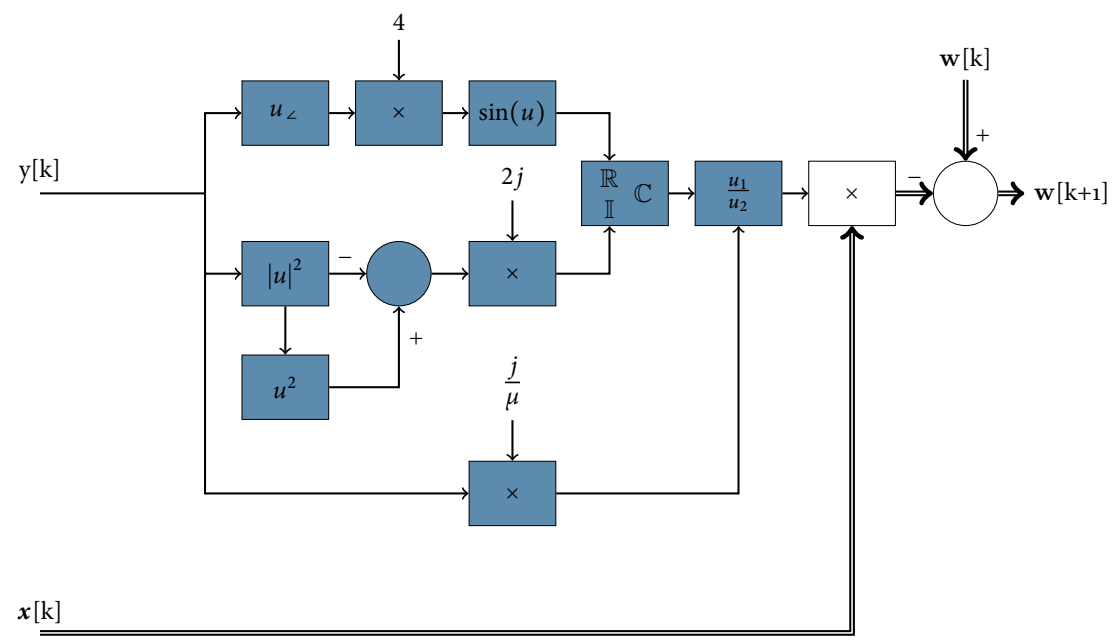

FIGURE 4.16 - Block diagram of the E-CMA algorithm.

\subsubsection{EMPIRICAL PERFORMANCE}

Similar to section 4.4.5, the E-CMA has been evaluated for empirical multi-channel data from the dive-center experiment (section 3.6.2).

\section{MSE performance}

Due to the different nature of the CMA and the E-CMA cost functions, it is difficult to evaluate these techniques merely in terms of cost behavior. In an attempt to compare their convergence performance, figure 4.17 shows the MSE estimates of the E-CMA output signal $(\mu=.25$ and $\mu=.5)$ and the MSE optimum. Since the E-CMA beamformer output is corrected for phase offsets by the structure of its cost criterion, it is not necessary to use the MSE calculation as specified in eq. 4.20. In contrast to the CMA learning curve illustrated in figure 4.14, the E-CMA seems to converge much faster to a lower MSE level. Evidently, the behavior of the ECMA for the different convergence factors exhibits almost similar performance. For robustness, it would therefore be wise to settle on the smallest convergence factor possible. In future work we would like to optimize the convergence factor on a symbol-to-symbol basis, analog to the techniques presented in [97]. 


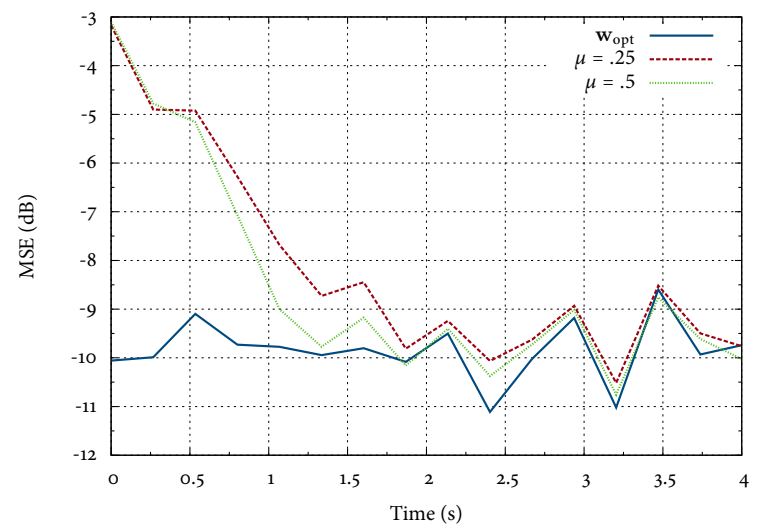

FIGURE 4.17 - MSE levels during application of the E-CMA.

\subsection{The ANgUlar CONSTANT MODULUS ALgORITHM}

Existing blind adaptive array algorithms operate on all signals from the distinct array elements and subsequently update the entire complex weight vector. This section elaborates on the angular CMA (A-CMA), a novel blind adaptive method that updates the steering angle instead of the entire weight vector. The A-CMA and its respective steering angle updates are particularly useful in the context of mixed-signal hierarchical arrays as a means to find and distribute steering parameters. In a mixed-signal hierarchical array, beamforming is performed on multiple levels, partly analog and partly digital. To a certain extent, spatial interference is already being suppressed by the analog beamformers before the signal is being digitized [75]. These analog beamformers decrease the dynamic range of the analog input signals and therefore lower the ADC requirements. Typically, less stringent ADC requirements yield a reduced power consumption, which is essential for battery limited underwater sensor nodes. After the sampling operation, remaining interference can be further suppressed or nulled, in the digital domain.

In a mixed-signal hierarchical array architecture, a classical digital blind beamforming algorithm has merely the results of the analog beamformers available and can therefore only update its own digital steering parameters. However, for deterministic steering of the complete hierarchical system, a novel adaptive algorithm is required that can efficiently track a desired signal and distribute steering parameters throughout the complete array. The proposed method, A-CMA, recognizes mispointing and calculates the required steering angle updates (instead of weight vector updates). The desired steering angle is calculated at the digital level of the hierarchical architecture and is thereafter distributed to both the analog and digital beamformers, as shown in figure 4.18. The top part of this figure (within the marked boxes) depicts the analog beamformers controlled by weights $\mathbf{w}_{a}$ and the 


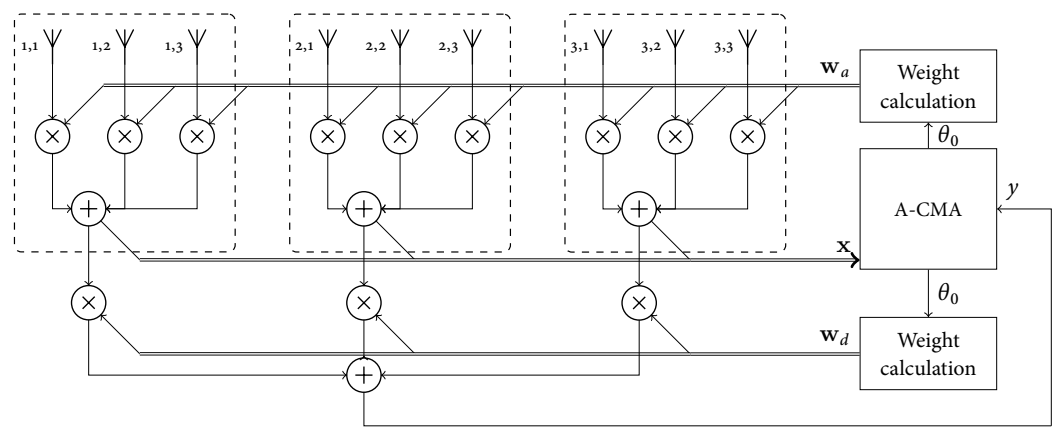

FIGURE 4.18 - Application of the A-CMA in a $3 \times 3$ mixed-signal hierarchical array.

bottom part depicts the digital beamformer controlled through weights $\mathbf{w}_{d}$. Our approach is based on the conventional CMA algorithm, but since it operates on the steering angle instead of the steering vector, it is called the A-CMA. Originally, this algorithm was designed for tracking a signal source which has a strong LoS arrival in an environment with practically no multipath, e.g., DVB-S signal tracking.

Both the CMA and the A-CMA use the CM property (section 4.4) of the received signal as a reference to calculate appropriate weight adjustments. The search space of the CMA constitutes of all complex weights that make up the steering vector [24]. However, in the A-CMA this search space is reduced to a single steering angle.

For analysis, this work explains the A-CMA in the context of a simplified (nonhierarchical) array such as shown in figure 4.19. Herein, the A-CMA operates on the narrowband signals received by an $N$-element ULA. The output of the ULA at each sample instant is the vector of $N$ quadrature baseband samples indicated by $\mathbf{x}$. A changing DoA angle affects the antenna data $\mathbf{x}$. The conventional CMA compensates for these effects by updating the steering vector $\mathbf{w}$ directly. The ACMA first calculates the desired steering angle $\theta_{0}$ and uses a linear phase taper (LPT) to determine the weights $\mathbf{w}$.

Cost function adjustments leading to the A-CMA and the actual derivation of the cost minimizer are discussed in section 4.6.1. Based on A-CMA's error-performance surface, its convergence properties are elaborated on in section 4.6.2. The computational complexity of the A-CMA is discussed in section 4.6.3. Section 4.6.4 compares simulation results of the A-CMA and the conventional CMA. An overview of the most significant results and ideas for future work are given in section 4.7.

\subsubsection{A-CMA COST CRITERION AND MINIMIZER}

The A-CMA cost criterion $J_{\mathrm{A}-\mathrm{CMA}}\left(\theta_{0}\right)$ can be constructed by reducing the $\mathrm{N}$ complex weights of the CMA criterion to a single steering angle $\theta_{0}$. A linear phase taper (LPT) is used to provide the mapping $\mathbf{w}\left(\theta_{0}\right)$ between the complex weights and the steering angle. The concept of an LPT is elaborated below. The cost minimizer for 


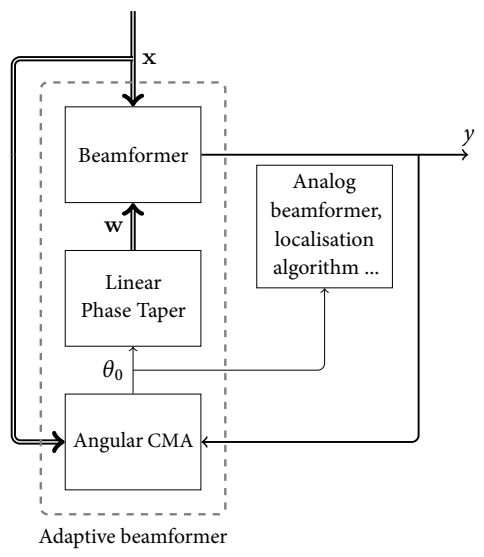

FIGURE 4.19 - Adaptive beamforming using the A-CMA.

the A-CMA cost function can be found by differentiating $J_{\mathrm{A}-\mathrm{CMA}}\left(\theta_{0}\right)$ with respect to the steering angle $\theta_{0}$, as explained in the end of this section.

\section{Linear Phase Taper}

An LPT is a linear phase variation across the array aperture. The weights of a conventional (narrowband) beamformer can be considered an LPT. Therefore, for a narrowband ULA, the LPT $\mathbf{w}\left(\theta_{0}\right)$ determines the array weights that exactly compensate the array response vector ${ }^{3} \mathbf{d}\left(\theta_{0}\right)$ (section 4.2.6):

$$
\mathbf{w}\left(\theta_{0}\right)=\mathbf{d}^{*}\left(\theta_{0}\right)=\left(\mathbf{v}\left(\theta_{0}\right) \otimes e^{-j \omega 0 T_{s}}\right)^{*}=\mathbf{v}^{*}\left(\theta_{0}\right) .
$$

Herein, assume that $\mathbf{v}\left(\theta_{0}\right)$ is the array manifold vector for an $N$-element ULA, with an element spacing of $d$, having the leftmost array element $\mathrm{p}_{0}$ positioned on the origin (eq. 4.6):

$$
\mathbf{v}\left(\theta_{0}\right)=e^{-j \frac{\omega}{c} d[0,-1, \ldots,-(N-1)]^{T} \cdot \mathbf{a}\left(\theta_{0}, 0\right)}=e^{j \frac{\omega}{c} d[0,1, \ldots,(N-1)]^{T} \cdot \mathbf{a}\left(\theta_{0}, 0\right)} .
$$

Since all array elements are located on the $\mathrm{x}$-axis, rewrite $\mathbf{a}\left(\theta_{0}, 0\right)$ in terms of its $x$-component $-u_{x}\left(\theta_{0}, 0\right)=-\sin \left(\theta_{0}\right)$ to find the LPT $\mathbf{w}\left(\theta_{0}\right)$ :

$$
\mathbf{w}\left(\theta_{0}\right)=\mathbf{v}^{*}\left(\theta_{0}\right)=e^{j \frac{\omega}{c} d \sin \left(\theta_{0}\right)[0,1, \ldots,(N-1)]^{T}}=e^{\psi\left(\theta_{0}\right) \mathbf{n}} .
$$

with $\psi\left(\theta_{0}\right)$ and $\mathbf{n}$ defined as, respectively:

$$
\psi\left(\theta_{0}\right)=j \frac{\omega}{c} d \sin \left(\theta_{0}\right), \quad \mathbf{n}=[0,1, \ldots,(N-1)]^{T} .
$$

Herein, $c$ represents the propagation speed of the incidence wave and $\omega$ the highest frequency (in $\mathrm{rad} \mathrm{s}^{-1}$ ) of the narrowband signal.

${ }^{3}$ A ULA can only change its directionality in the xz-plane. Therefore, the azimuth angle $\phi$ in the weight and the array response vector is dropped. 
A cost minimizer for the A-CMA can be found by expressing $y$ in eq. 4.16 as $\mathbf{w}\left(\theta_{0}\right)^{H} \mathbf{x}=\left(e^{\psi\left(\theta_{0}\right) \cdot \mathbf{n}}\right)^{H} \mathbf{x}$. Thereafter, the first derivative of $J_{\mathrm{A}-\mathrm{CMA}}$ with respect to $\theta_{0}$ is determined:

$$
\begin{aligned}
J_{\mathrm{A}-\mathrm{CMA}}\left(\theta_{0}\right) & =E\left\{\left(\left|\left(e^{\psi\left(\theta_{0}\right) \cdot \mathbf{n}}\right)^{H} \mathbf{x}\right|^{2}-1\right)^{2}\right\} \\
\frac{\delta}{\delta \theta_{0}} J_{\mathrm{A}-\mathrm{CMA}} & =E\left\{2\left(|y|^{2}-1\right) \cdot \frac{\delta}{\delta \theta_{0}}\left(\left(e^{\psi\left(\theta_{0}\right) \cdot \mathbf{n}}\right)^{H} \mathbf{x x}^{H} e^{\psi\left(\theta_{0}\right) \cdot \mathbf{n}}-1\right)\right\} \\
& =2 E\left\{\left(|y|^{2}-1\right) \cdot \frac{\delta}{\delta \theta_{0}}\left(\mathbf{x}^{H} e^{\psi\left(\theta_{0}\right) \cdot \mathbf{n}}\left(e^{-\psi\left(\theta_{0}\right) \cdot \mathbf{n}}\right)^{T} \mathbf{x}-1\right)\right\} .
\end{aligned}
$$

Continue by writing $e^{\psi\left(\theta_{0}\right) \cdot \mathbf{n}}\left(e^{-\psi\left(\theta_{0}\right) \cdot \mathbf{n}}\right)^{T}$ as matrix $\mathbf{B}$ :

$$
\frac{\delta}{\delta \theta_{0}} J_{\mathrm{A}-\mathrm{CMA}}=2 E\left\{\left(|y|^{2}-1\right) \cdot \frac{\delta}{\delta \theta_{0}}\left(\mathbf{x}^{H} \mathbf{B} \mathbf{x}-1\right)\right\} .
$$

with B,

$$
\mathbf{B}=\left[\begin{array}{ccc}
1 & \cdots & e^{\psi\left(\theta_{0}\right) \cdot\left(n_{0}-n_{N-1}\right)} \\
\vdots & 1 & \vdots \\
e^{\psi\left(\theta_{0}\right) \cdot\left(n_{N-1}-n_{0}\right)} & \cdots & 1
\end{array}\right]
$$

The derivative of $J_{\mathrm{A}-\mathrm{CMA}}$ with respect to $\theta_{0}$ is now written in eq. 4.35 .

$$
\frac{\delta}{\delta \theta_{0}} J_{\mathrm{A}-\mathrm{CMA}}=2 E\left\{\left(|y|^{2}-1\right) \cdot\left(\mathbf{x}^{H} \mathbf{B}^{\prime}\left(\theta_{0}\right) \mathbf{x}\right)\right\}
$$

where $\mathbf{B}^{\prime}\left(\theta_{0}\right)=\frac{\delta}{\delta \theta} \mathbf{B}\left(\theta_{0}\right)$ can be written as:

$$
\left[\begin{array}{ccc}
0 & \cdots & \left(n_{0}-n_{N-1}\right) \psi^{\prime} e^{\left(n_{0}-n_{N-1}\right) \psi} \\
\vdots & 0 & \vdots \\
\left(n_{N-1}-n_{0}\right) \psi^{\prime} e^{\left(n_{N-1}-n_{0}\right) \psi} & \cdots & 0
\end{array}\right] .
$$

Herein, $\psi^{\prime}\left(\theta_{0}\right)$ is $\frac{\delta}{\delta \theta_{0}} \psi\left(\theta_{0}\right)$ :

$$
\psi^{\prime}\left(\theta_{0}\right)=j \frac{\omega}{c} d \cos \left(\theta_{0}\right)
$$

The use of an instantaneous gradient estimate for eq. 4.35 results in the following (gradient descent) cost minimizer for $J_{\mathrm{A}-\mathrm{CMA}}$ :

$$
\theta_{0}[k+1]=\theta_{0}[k]-\mu\left(|y[k]|^{2}-1\right) \cdot\left(\mathbf{x}[k]^{H} \mathbf{B}^{\prime}\left(\theta_{0}[k]\right) \mathbf{x}[k]\right)
$$

Herein, the value 2 is absorbed in the convergence factor $\mu$. 


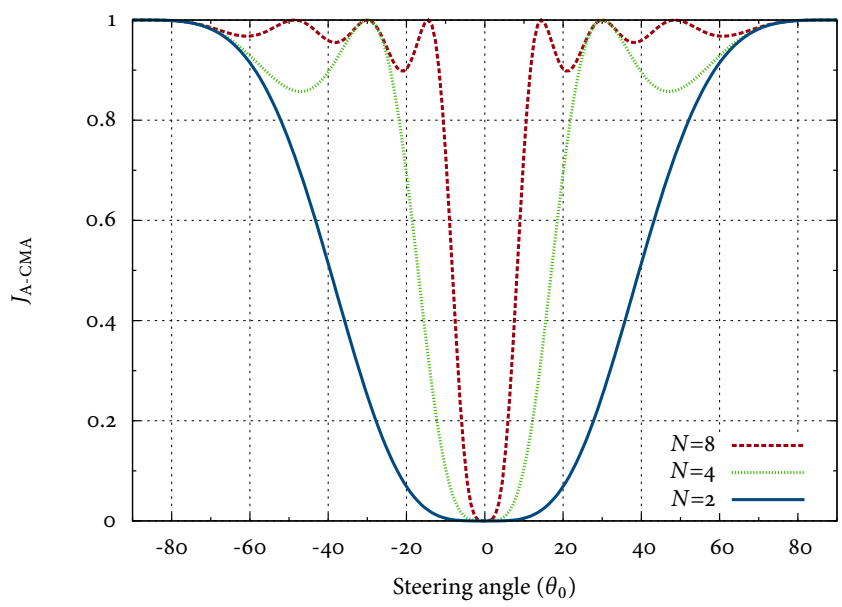

FIGURE 4.20 - Error-performance surface of the A-CMA.

\subsubsection{ERROR-PERFORMANCE SURFACE}

The dependence of a cost function on its weights is called the error-performance surface [26]. For the CMA, this dependence is an $(N+1)$-dimensional surface with $N$ degrees of freedom, where $N$ is the number of array elements. In the case of more than two degrees of freedom, such a surface is hard to visualize.

The error-performance surface of the A-CMA is two-dimensional with only one degree of freedom (the steering angle $\theta_{0}$ ). The A-CMA's error-performance can be written as:

$$
J_{\mathrm{A}-\mathrm{CMA}}\left(\theta_{0}\right)=\left(\left|\mathbf{w}\left(\theta_{0}\right)^{H} \mathbf{x}\right|^{2}-1\right)^{2}=\left(\left|\left(e^{\psi\left(\theta_{0}\right) \cdot \mathbf{n}}\right)^{H} \mathbf{x}\right|^{2}-1\right)^{2}
$$

Herein, the expectation operator ' $E$ ' is dropped because $\mathbf{x}$ is assumed to be array data for a single source with no additive noise.

Figure 4.20 shows the error-performance surface of the A-CMA for an element spacing of $\frac{\lambda}{2}$ and signals arriving from broadside. The error-performance is drawn for a two-, four- and eight-element array. Since impinging signals arrive from broadside, for all three array configurations, the steering angle $\theta_{0}$ equal to zero has the lowest costs.

Based on figure 4.20, one can recognize that the error-performance surface of the A-CMA has the appearance of a vertically flipped array pattern. An increase in the number of array elements results in a smaller angular range where convergence to the global minimum can be guaranteed. The convergence region corresponds to the INBW of an array pattern (section 4.2.7). The INBW of the main beam can be expressed as follows:

$$
\mathrm{INBW}=2 \sin ^{-1}(\lambda /(d N))
$$


For example, for an eight-element array with $\frac{\lambda}{2}$ element spacing, the A-CMA has a convergence region width of $2 \sin ^{-1}\left(\frac{1}{4}\right) \approx 29^{\circ}$.

DoA estimation algorithms, such as MUSIC, can be used to provide an initial steering angle for the A-CMA [70]. The accuracy of this estimate should be within the angular range that provides convergence to the global minimum.

\subsubsection{COMPLEXITY ANALYSIS}

A short analysis on the complexity of the A-CMA is given to assess scalability and implementability. The computational complexity of the A-CMA is compared with the complexity of the CMA and MUSIC. MUSIC is included in the comparison because it provides DoA estimates of all impinging signals. Therefore, repeated execution of MUSIC could be used for tracking. However, this approach requires a method to identify the DoA of the desired signal out of all angles found by MUSIC.

The computational order of complexity of the algorithms is determined by counting the number of complex MAC operations. The complexity of the A-CMA is determined based on eq. 4.36. Calculation of matrix $\mathbf{B}^{\prime}\left(\theta_{0}[k]\right)$ is kept out of the complexity analysis since we expect that, by exploiting its Hermitian symmetry, this can be done very efficiently. Therefore, not taking into account calculation of $\mathbf{B}^{\prime}\left(\theta_{0}[k]\right)$, the order of the A-CMA is dominated by the matrix-vector multiplication $\mathbf{B}^{\prime}\left(\theta_{0}[k]\right) \mathbf{x}[k]$ and is for that reason $\Theta\left(N^{2}\right)$. Herein, $N$ is the number of array elements.

The conventional gradient-descent version of the CMA is of order $\Theta(N)$ [97]. MUSIC is computationally much more expensive with a complexity of order $\Theta\left(N^{3}\right)$ [67]. Thus, the complexity of the A-CMA is in between that of the CMA and MUSIC.

\subsubsection{SIMULATION RESULTS}

The A-CMA has also been applied to empirical data from the dive-center experiment (section 3.6.2). Given this input data, the algorithm initially converges into the direction of the expected LoS angle. However, the A-CMA is restricted to array responses generated by an LPT. Therefore, the positions of the nulls are coupled to the steering angle $\theta_{0}$. This limited freedom did not result in practically useful MSE levels. Therefore, the convergence of the A-CMA is examined by looking at its cost and MSE behaviour for synthetic input data. To investigate whether the A-CMA is practical in less reflective underwater environments is part of future work.

Simulations are performed for both the A-CMA and the conventional CMA to provide means for comparison. For simulation, the adaptive beamformer architecture presented in figure 4.19 is implemented. An eight-element ULA with a half-wavelength element spacing is assumed. The synthetic baseband samples $\mathbf{x}$ are based on a single QPSK-modulated source with additive noise (SNR of $16 \mathrm{~dB}$ ). 


\section{Learning curve}

Similar to analysis of the CMA (section 4.4), the performance of the A-CMA is studied by looking at the learning curve of its cost function. As mentioned in section 4.6.2, an eight-element array executing the A-CMA should be able to converge to the correct array steering angle if the DoA of the impinging signal stays within the $29^{\circ}$ wide convergence region. Validity of this statement is checked in simulation by setting the DoA angle to broadside $\left(0^{\circ}\right)$, while having the initial steering angle set to one of the extremes of the convergence region $\left(0^{\circ} \pm \frac{29}{2} \approx \pm 14^{\circ}\right)$. Figure 4.21 shows that the A-CMA rapidly convergences to the correct steering angle and consequently minimizes the costs. The simulation uses a convergence factor $\mu$ of $5 \cdot 10^{-2}$. The A-CMA is still robust with a large convergence factor, because the gradient-descent ensures global minimization if the steering angle is initially within the convergence region.

To compare the convergence properties of the A-CMA with the CMA, the cost behaviour of the CMA, for similar initial weights and the same convergence factor, is also illustrated in figure 4.21. Clearly, the CMA takes more samples to converge than the A-CMA. The rapid convergence of the A-CMA is caused by its drastic dimensionality reduction from $N$ complex weights to a single real value.

\section{Mean square error}

The estimates are based on the same scenario as in the previous simulation. Since we are only interested in revealing trends, the results are smoothed by a moving average filter. Initially, the MSE level of the A-CMA after convergence is below that of the CMA. Asymptotically, the MSE level of the CMA approaches the MSE level of the A-CMA.

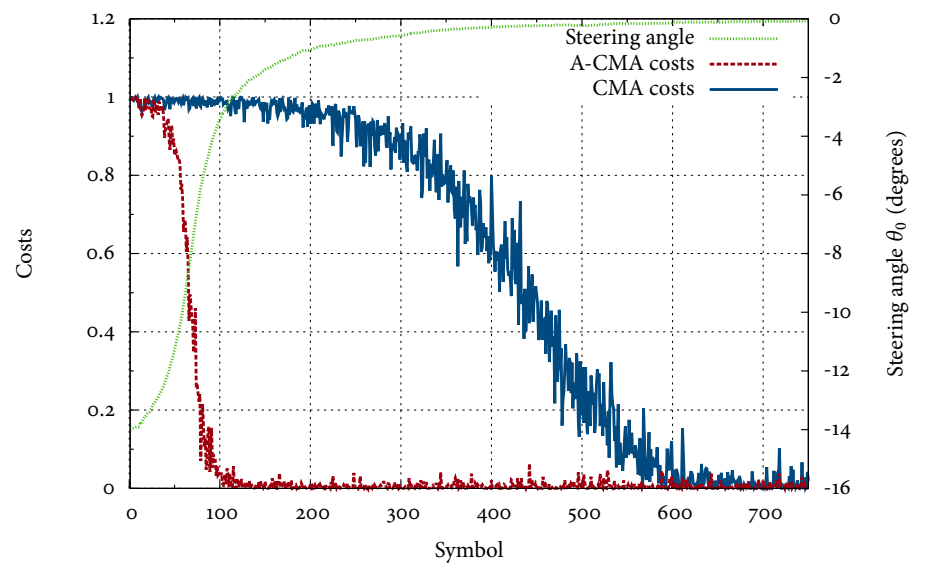

FIGURE 4.21 - Convergence behaviour of the CMA and the A-CMA $\left(\mu=5 \cdot 10^{-2}\right)$. 


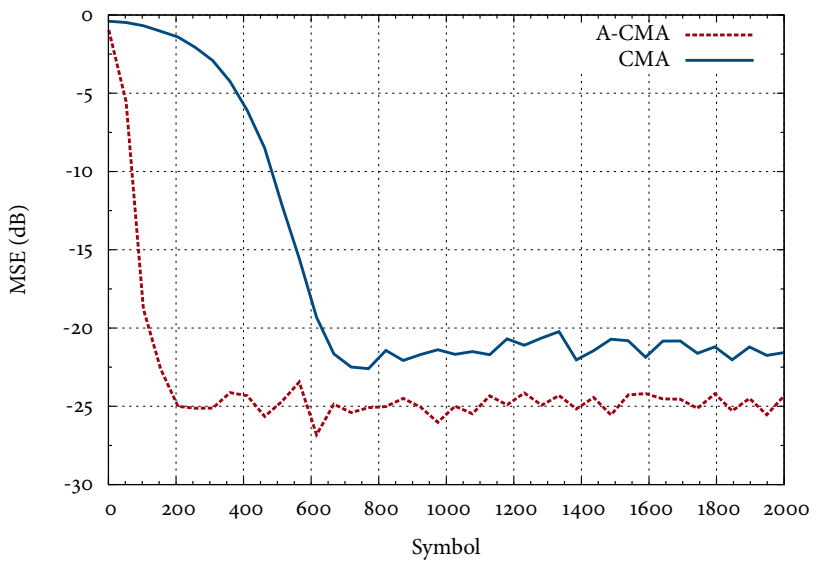

FigURE 4.22 - MSE comparison $\left(\mu=5 \cdot 10^{-2}\right)$.

\subsection{CONCLUSIONS AND FUTURE WORK}

In this chapter, techniques for mitigation of signal distortion in the spatial dimension were presented. Within this general class, we limited our focus to linear combinations of spatially distinct samples, whose coefficients are known as array weights. To reduce energy consumption, structural properties of the transmitted signal (instead of training sequences) were exploited to appropriately update the array weights. The latter is known as blind spatial equalization.

To achieve a (relatively) high spectral efficiency, while being power-efficient, our underwater transmissions are QPSK modulated. The output of a QPSK modulator always has a constant modulus (CM). A well-known blind equalization method that uses this CM property, the CMA, has been extensively discussed and applied to multi-channel data from the dive-center experiment (section 3.6.2). CMA's empirical performance reveals, even for relatively large convergence factors, asymptotic convergence to the optimum array weights.

The CMA does not compensate for phase deviations. Therefore, the E-CMA is studied. The E-CMA is a stricter blind narrowband method, which exploits both the CM property and the equal phase distribution of QPSK signals to optimize array weights. This blind method has never been studied in a spatial (underwater) context. In our empirical evaluation, the MSE levels of the E-CMA seem to converge much faster than those of the CMA. However, we do note that our empirical data was captured in a reverberant, but relatively time-invariant environment (ten meter deep water tank). Further research should investigate performance of the E-CMA in more dynamic environments. 
Additionally, a rather unconventional method for blind equalization, known as the A-CMA, has been presented in this chapter. In contrast to conventional blind equalization methods, it calculates steering angle updates instead of weight vector updates. Originally this algorithm was designed for tracking a single source that has a strong LoS arrival in an environment with practically no multipath. Effectively, our approach reduces the dimensionality of the CMA's search space from $N$ complex weights (number of array elements) to a single real value. We have illustrated that this approach is attractive in mixed-signal hierarchical arrays, e.g., to lower ADC requirements. Though in this thesis, the A-CMA was examined within the context of a simplified non-hierarchical beamformer. Further research should look at the applicability of the algorithm in a hierarchical setup in simulation and in practice.

Based on synthetic data, the cost behavior of the A-CMA has been compared with that of the CMA. Since the A-CMA is restricted to array responses generated by a linear phase taper (LPT), it cannot freely position nulls or correct for small array imperfections. Nevertheless, the A-CMA provides faster convergence and a slightly lower MSE floor. 



\title{
5 \\ SPECTRAL EQUALIZATION
}

\begin{abstract}
The propagation speed of underwater acoustic pressure waves is variable. Therefore, a direct-path acoustic wave can arrive later than a reflected wave. This phenomenon, known as nonminimum-phase behaviour, complicates blind extraction of a channel's phase response. Blind equalization of the magnitude and phase response of a nonminimum-phase channel can always be performed separately, because the equalizer of a nonminimum-phase channel is decomposable into a (i) minimum-phase and an (ii) all-pass part.
\end{abstract}

This chapter introduces an approach, called the all-pass CMA, that reduces the dimensionality of the CMA algorithm to improve blind equalization of a channel's all-pass part. The dimensionality reduction has been performed by parameterizing the CMA cost function in terms of the nonminimumphase zero location of the all-pass part to be compensated. The all-pass $C M A$ can compensate a single nonminimum-phase zero. Compared to the CMA, it typically provides a faster and more accurate compensation of this zero.

\subsection{INTRODUCTION}

The previous chapter discusses the primary dimensions of the channel and the transmitted signal: time, space and frequency. In the multipath-rich underwater environment, energy spillovers in the time dimension (reflections from current and previous transmissions) add up constructively and destructively, producing frequency-selective channel distortion. Various techniques exist for mitigation of frequency-selective channel distortion, e.g., spectral equalization, spread spectrum (SS) modulation and multi-carrier modulation [73]. This chapter elaborates on the spectral counterpart of spatial equalization, known as spectral equalization. Spectral equalization to deal with the effects of multipath propagation is a major challenge in the design of underwater acoustic communication systems. In this thesis, we have chosen to focus on spectral and spatial equalization. This approach is advantageous, since spectral and spatial methods have many mathematical analogies. It is likely

Parts of this chapter have been published in [KCH:3] . 
that a method with promising performance in one of these domains can easily be transformed to the other domain.

In the underwater environment, the source, the scatterers, the medium and the receiver can be moving. Similar to adaptive equalization in the spatial dimension, also the weights of a spectral equalizer need to be adjusted continuously. To perform these adjustments, adaptive spectral equalizers can be employed. Ideally, an adaptive spectral equalizer is a filter that, in combination with the (time-varying) channel response, approximates the identity operation. For similar reasons as in chapter 4; (i) our transmissions are QPSK modulated and (ii) to find appropriate weight updates, we focus on blind adaptive equalization. Blind adaptive spectral equalization and the criterion for ideal spectral equalization are briefly discussed in section 5.2.1.

A realistic channel response, such as the response in figure 2.8 , can be modeled by a finite impulse response (FIR). For ideal equalization of the distortion caused by a FIR, an infinite impulse response (IIR) is needed. Depending on the $(z \text {-domain })^{1}$ zero positions of the FIR, a finite-length approximation of the IIR can be used for equalization. Long FIR equalizers, having a large computational complexity, are needed for channels with zeros close to the unit circle.

As previously discussed in section 2.5.4, underwater channel responses can be minimum-phase or nonminimum-phase. A $z$-domain discrete-time channel model is characterized as nonminimum-phase if at least one of its $z$-plane zeros is located outside the unit circle. For all channels with a similar magnitude response, the minimum-phase system has (most of) its energy concentrated near the start of the impulse response [64]. For a minimum-phase system, there is a one-to-one relation between the magnitude response and the phase response [52]. Such a relationship does not exists for a nonminimum-phase channel. Therefore, given only the magnitude response of a nonminimum-phase channel, its phase response cannot uniquely be identified.

Since many blind equalization techniques cannot determine the correct phase response of nonminimum-phase channels, equalization of these channels is more difficult than equalization of minimum phase channels. This apparent phase blindness is caused by the fact that a lot of equalizers are based on SOS (e.g., autocorrelation) and reasoning in the autocorrelation domain suppresses phase information [52]. SOS-based equalization applied to a nonminimum-phase channel produces an equalizer for the spectrally equivalent minimum phase (SEMP) version of the channel, but it leaves the phase response uncorrected.

To blindly extract the phase response of nonminimum-phase channels, (i) methods based on higher-order statistics (HOS) or (ii) techniques exploiting cyclostationarity can be used [52]. Cyclostationarity is a property used to designate stochastic

\footnotetext{
${ }^{1}$ Note that the symbol $z$ is reused. In chapter 2, as in other literature on underwater acoustics [86], $z$ represents depth. In this chapter, $z$ refers to the complex variable used in the power series of the $z$-transform.
} 
processes whose statistical properties vary periodically over time [22]. Exploiting cyclostationarity requires an equalizer input which is sampled faster than the symbol rate. Since we assume symbol-rate spaced equalization, methods based on cyclostationarity are not taken into consideration. Similar to chapter 4 , in this chapter, HOS-based methods are employed to find appropriate weight updates. Fundamental theorems on the identifiability of nonminimum-phase channels by application of HOS methods are discussed in appendix A.

Blind equalization of the magnitude and phase response of a nonminimum-phase channel can be performed separately because a nonminimum-phase channel equalizer can always be decomposed into a minimum-phase and an all-pass part. To reduce the computational complexity of a nonminimum-phase channel equalizer, Da Roche et al. [16] proposed an equalizer based on a cascade of an (computationally less complex) IIR filter and an all-pass response approximated by an FIR filter. The responsibility of the IIR filter is compensation of the channel's magnitude response, whereas the all-pass filter equalizes the phase. To equalize the phase, the all-pass filter weights are estimated by minimizing a direct decision (DD) error criterion. A further reduction of the computational complexity of this cascaded equalizer structure can be found in the work of Lambotharan and Chambers [38]. Herein, the computational complexity of the equalizer is reduced by splitting the all-pass filter into an IIR and a FIR filter. In their approach, the FIR filter weights are estimated using a normalized version of the CMA algorithm.

Apart from computational complexity, another important consideration for application of adaptive equalizers is their convergence time. The convergence time should be small with respect to the coherence time of the channel. This chapter does not focus on the complexity of equalization structures, as in $[16,38]$, but addresses accelerating and improving blind estimation of the filter weights that mitigate the channel's phase response. To provide fast and accurate compensation for the distortion caused by a nonminimum-phase channel, we exploit prior knowledge of the mathematical structure of the possible equalizer weights to reduce the dimensionality of this blind estimation problem. Our technique is based on the CMA, but confines the CMA's solution space to merely all-pass filters and is therefore called the all-pass CMA (AP-CMA).

A theoretical background on equalization of nonminimum-phase channels using a cascade of a magnitude and a phase equalizer is given in section 5.2. Section 5.3 elaborates on our proposed dimensionality reduction of the conventional CMA to confine its solution space. The actual derivation of the AP-CMA, using Wirtinger calculus, is discussed in section 5.4. Herein, to fully focus on the algorithm's convergence behaviour instead of (potential) instability, the derivation only considers FIR filters. A comparison of the conventional CMA and the AP-CMA for equalization of the channel's phase response can be found in section 5.5. Conclusions and ideas for future work are discussed in section 5.6. 


\subsection{ThEORETICAL BACKGROUND}

In this section, we shortly elaborate on blind adaptive equalization and the decomposition and equalization of nonminimum-phase channels.

\subsubsection{BLIND ADAPTIVE EQUALIZATION}

Consider the structure of the blind adaptive equalizer shown in figure 5.1. The equalizer $W(z)$ mitigates time-varying frequency-selective distortion $H(z)$ in the received signal $x[k]$ based on structural (and related statistical) properties of the non-Gaussian source signal $s[k]$. The additive white gaussian noise (AWGN) is represented by $v[k]$. In further analysis, $v[k]$ will be ignored, because usually the ISI caused by $H(z)$ dominates the degradation of the received signal [26].

\section{Ideal equalization criterion}

Ideal equalization is attained if the aggregate channel response $P(z)(=H(z) \cdot W(z))$ approximates the identity operation. Nevertheless, the equalizer is allowed to impose a constant delay $d_{\mathrm{eq}}$ and a complex gain $c_{\mathrm{eq}}$ to the output signal $y[k]$ [52]:

$$
Z^{-1}\{P(z)\}=p[m]=\delta_{k}\left[m-d_{\mathrm{eq}}\right] \cdot c_{\mathrm{eq}} .
$$

Herein, $m$ is the index for the filter taps, $Z^{-1}$ is the inverse $z$-transform and $\delta_{k}$ represents the Kronecker delta function.

\subsubsection{MINIMUM-PHASE/ALL-PASS DECOMPOSITION}

A nonminimum-phase channel has at least one zero outside the unit circle and can always be decomposed into a minimum-phase and an all-pass part [64]. To find its minimum-phase part ${ }^{2}$, the zero(s) of a nonminimum-phase transfer located outside the unit circle, have to be reflected inside the unit circle to their conjugate reciprocal location(s). For a single-pole single-zero nonminimum-phase channel $H_{\mathrm{NMP}}(z)$, this minimum-phase/all-pass decomposition is shown in figure 5.1. Herein, $H_{\mathrm{NMP}}(z)$ is factored into its minimum-phase part $H_{\mathrm{MP}}(z)$ and its all-pass part $H_{\mathrm{AP}}^{a}(z) . H_{\mathrm{AP}}^{a}(z)$ is the all-pass channel with a zero at location $a$. Mathematically, the decomposition is given as:

$$
\begin{aligned}
H_{\mathrm{NMP}}(z) & =n_{0} \cdot \frac{(z-a)}{(z-b)} \\
& =n_{0} \cdot \frac{\left(z-\frac{1}{a^{*}}\right)}{(z-b)} \cdot \frac{(z-a)}{\left(z-\frac{1}{a^{*}}\right)}=H_{\mathrm{MP}}(z) \cdot H_{\mathrm{AP}}^{a}(z) .
\end{aligned}
$$

Herein, $a$ and $b$ are the zero and pole location of the nonminimum-phase channel. In section 5.3, the gain factor $n_{0}$ is used for normalization of the response.

\footnotetext{
${ }^{2}$ The minimum-phase part of a nonminimum-phase channel is known as its SEMP channel.
} 
By definition, a minimum-phase channel always has a causal and stable inverse (since its zeros are located inside the unit circle). In figure 5.1, the inverse of $H_{\mathrm{MP}}(z)$ is denoted $W_{\mathrm{MP}}(z)$. The stable and causal time-domain characterization of $W_{\mathrm{MP}}(z)$, the impulse response $w_{\mathrm{MP}}[\mathrm{m}]$, can be used to equalize the magnitude response of the channel.

The inverse of the channel's all-pass part $H_{\mathrm{AP}}^{a}(z)$ is known as $W_{\mathrm{AP}}^{a}(z)$. The inverse $\mathrm{z}$-transform of $W_{\mathrm{AP}}^{a}(z)$ is only stable if the region-of-convergence (ROC) is chosen to extend inward from the outermost pole, which results in the anti-causal timedomain characterization $w_{\mathrm{AP}}^{a}[m]$. Consequently, in order to equalize the channel's phase transfer $H_{\mathrm{AP}}^{a}(z)$, an anti-causal response (for example approximated by an FIR) or a block-based time reverser are required [16, 38].

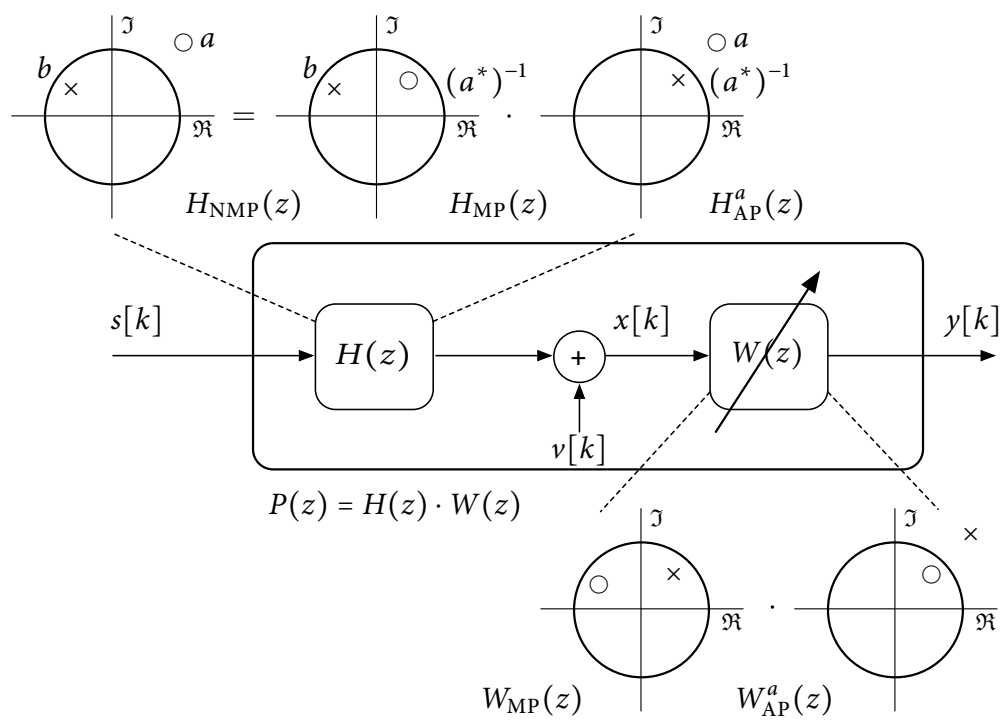

FIGURE 5.1 - Structure of a blind adaptive equalizer and the decomposition of a (single-pole single-zero) nonminimum phase channel.

\subsection{Dimensionality Reduction OF THE CMA}

Phase equalization requires an all-pass magnitude response. In this section, we exploit this knowledge to narrow the search space of the CMA to (single-pole single-zero) all-pass filters by reducing the dimensionality of the parameters to be estimated.

The time-domain expressions of a single-pole single-zero all-pass channel and equalizer are determined in section 5.3.1. In section 5.3.2 this expression is used 
to reduce the dimensionality of the conventional CMA. Section 5.3.3 discusses the error-performance surface of the all-pass CMA.

\subsubsection{ALL-PASS CHANNEL AND EQUALIZER}

\section{All-pass channel time-domain characterization}

To determine the unit magnitude and causal time-domain characterization of the channel's all-pass part $H_{\mathrm{AP}}^{a}(z)$, first its numerator and denominator are divided by $z$ to write the transfer in a more common form:

$$
H_{\mathrm{AP}}^{a}(z)=n_{0} \cdot \frac{z-a}{z-\frac{1}{a^{\star}}}=n_{0} \cdot \frac{1-a z^{-1}}{1-\frac{1}{a^{\star}} z^{-1}} .
$$

Thereafter, $n_{0}$ is set to $1 / a^{*}$ to normalize the magnitude response ${ }^{3}$. The unit magnitude and causal $z$-domain characterization of $H_{\mathrm{AP}}^{a}(z)$ can now be written as (ROC: $\left.|z|>\left|1 / a^{*}\right|\right)$ :

$$
H_{\mathrm{AP}}^{a}(z)=\frac{1}{a^{\star}} \frac{1-a z^{-1}}{1-\frac{1}{a^{\star}} z^{-1}}=\frac{1-a z^{-1}}{a^{*}-z^{-1}} .
$$

Subsequently, long division of the numerator over the denominator results in:

$$
H_{\mathrm{AP}}^{a}(z)=a+\frac{1-a a^{*}}{a^{*}-z^{-1}}
$$

The $M$-tap causal time-domain approximation of $H_{\mathrm{AP}}^{a}(z)$ is the discrete-time impulse response $\mathbf{h}_{\mathrm{AP}}^{a}$. Given eq. 5.2, the response $\mathbf{h}_{\mathrm{AP}}^{a}$ can be found by consulting the $z$-transform pair table in [64]:

$$
\mathbf{h}_{\mathrm{AP}}^{a}=a \cdot \delta_{k}[m]+\left(\frac{1}{a^{*}}-a\right)\left(\frac{1}{a^{*}}\right)^{m} u[m], \quad m=0, \ldots,(M-1) .
$$

Herein, $u[m]$ is the discrete-time unit step function. Note that, $m$ is an index for the filter taps.

\section{All-pass equalizer time-domain characterization}

To compensate for the effects of $H_{\mathrm{AP}}^{a}(z)$, a time-domain characterization of its inverse is used. Similar to the previous section, derivation of this time-domain characterization requires long division, followed by consulting the $z$-transform pair table in [64].

As explained in section 5.2.3, to guarantee stability, the time-domain characterization of $H_{\mathrm{AP}}(z)^{-1}$ must be anti-causal. The $M$-tap anti-causal time-domain approximation $\mathbf{w}_{\mathrm{AP}}^{a}$ of the (single-pole single-zero) all-pass equalizer $W_{\mathrm{AP}}^{a}(z)$ can be

\footnotetext{
${ }^{3}$ For verification, evaluate the frequency response $H_{\mathrm{AP}}^{a}(z)_{\mid z=e^{j \omega}}$ at $\omega=0$.
} 
found as follows (ROC: $|z|<|a|$ ):

$$
\begin{aligned}
\mathbf{w}_{\mathrm{AP}}^{a} & =Z^{-1}\left\{\frac{a^{*}-z^{-1}}{1-a z^{-1}}\right\} \\
& =Z^{-1}\left\{\frac{1}{a}+\frac{a^{*}-a^{-1}}{1-a z^{-1}}\right\} \\
& =\frac{\delta[m]}{a}-\left(a^{*}-\frac{1}{a}\right) a^{m} u[-m-1], \quad m=(-M+1), \ldots, 0 .
\end{aligned}
$$

Since we are only dealing with all-pass transfers in the rest of this chapter, for notational convenience and clarity, $\mathbf{h}_{\mathrm{AP}}^{a}$ and $\mathbf{w}_{\mathrm{AP}}^{a}$ will be written as $\mathbf{h}(a)$ and $\mathbf{w}(a)$.

\subsubsection{SINGLE- POLE SINGLE-ZERO ALL-PASS CMA}

The cost criterion of the CMA, which is a measure for the average deviation of the equalizer output $y$ from a constant modulus, was extensively discussed in section 4.4 .

In the context of spectral equalization, the expected CMA costs (over time) can be written in terms of the equalizer weights $\mathbf{w}$ as follows:

$$
J_{\mathrm{CMA}}(\mathbf{w})=E\left\{\left[\left|\mathbf{w}^{T} \mathbf{x}\right|^{2}-R_{2}\right]^{2}\right\}, \quad \mathbf{x}=x[k, \ldots, k+M-1]^{T},
$$

where $k$ is an index for the sampling instants and $R_{2}$ is the Godard parameter. As in chapter 4, the Godard parameter is set to one. Note that, the spectral equalizer output $y$ is written as $\mathbf{w}^{T} \mathbf{x}$ instead of $\mathbf{w}^{H} \mathbf{x}$. In spatial equalization or beamforming it is convenient to use the Hermitian inner product. When the Hermitian form is used then, in case of conventional beamforming, the weight vector equals the array response vector.

The conventional CMA tries to minimize the deviation from a constant modulus by choosing an appropriate weight vector w. In this work, the dimensionality of the CMA is reduced by substituting w with $\mathbf{w}(\hat{a})$, where $\hat{a}$ is the (estimated) zero position compensated by the equalizer. Note that this substitution is only valid if the CMA is used for (single-pole single-zero) phase equalization. Effectively, the dimensionality-reduced cost function $J_{\mathrm{AP}-\mathrm{CMA}}$ is a function of the zero position $\hat{a}$ compensated by the equalizer:

$$
J_{\mathrm{AP}-\mathrm{CMA}}(\hat{a})=E\left\{\left[\left|\mathbf{w}(\hat{a})^{T} \mathbf{x}\right|^{2}-1{ }^{2}\right\}, \quad \mathbf{x}=x[k \ldots k+M-1]^{T} .\right.
$$

In contrast to an $M$-tap conventional CMA equalizer, the dimensionality of the optimization problem is now reduced from $M$ complex weights to a single complex weight $\left(\mathbb{C}^{M} \rightarrow \mathbb{C}\right)$. 
The dependence of a cost function on its weight(s) is called the error-performance surface (section 4.6.2). For the AP-CMA, this surface is written as $J_{\mathrm{AP} \text { - CMA }}^{a}(\hat{a})$, where $a$ is the zero position in the nonminimum-phase channel and $\hat{a}$ is the zero position compensated by the equalizer.

Since $J_{\mathrm{AP} \text { - CMA }}^{a}(\hat{a})$ is of type $\mathbb{C} \rightarrow \mathbb{R}$, the error-performance surface of the APCMA can be visualized in a three-dimensional surface plot. The error-performance surface can be written as follows:

$$
\begin{aligned}
J_{\mathrm{AP}-\mathrm{CMA}}^{a}(\hat{a}) & =E\left\{\left[\left|\mathbf{w}(\hat{a})^{T} \mathbf{x}\right|^{2}-1\right]^{2}\right\} \\
\mathbf{x} & =\left[\begin{array}{c}
\mathbf{h}(a)^{T} s[k, \ldots, k-M+1] \\
\mathbf{h}(a)^{T} s[(k+1), \ldots,(k+1)-M+1] \\
\vdots \\
\mathbf{h}(a)^{T} s[(k+M-1), \ldots,(k+M-1)-M+1]
\end{array}\right] .
\end{aligned}
$$

Herein, the received signal $\mathbf{x}$ is generated by filtering a sequence of uniform independent and identically distributed (i.i.d.) zero-mean QPSK symbols $s[k]$ with response $\mathbf{h}(a)$. Column vector $\mathbf{h}(a)$ represents an $M$-tap time-domain approximation of the channel's all-pass response. Therefore, the values in $\mathbf{x}$ can be considered as output samples of a moving average (MA) process.

The error-performance surface $J_{\mathrm{AP} \text { - CMA }}^{1+1 j}(\hat{a})$ for the channel $\mathbf{h}(1+1 j)$ is shown in figure 5.2. Both $\mathbf{h}(1+1 j)$ and $\mathbf{w}(\hat{a})$ are calculated for eight-order FIR filters $(M=9)$. It can clearly be seen that the costs are minimized if the (ideal) equalizer $\mathbf{w}(1+1 j)$

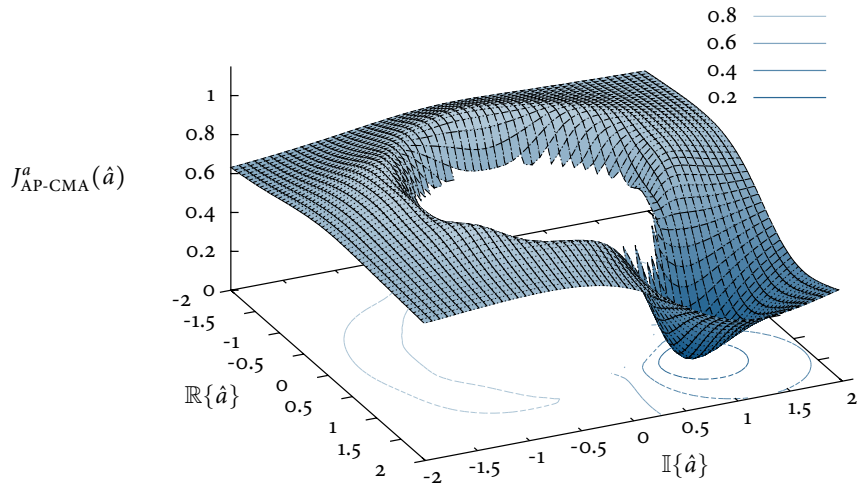

FigURE 5.2 - Error-performance surface $J_{\mathrm{AP}-\mathrm{CMA}}^{1+1 j}(\hat{a})(\mathrm{M}=9)$. 


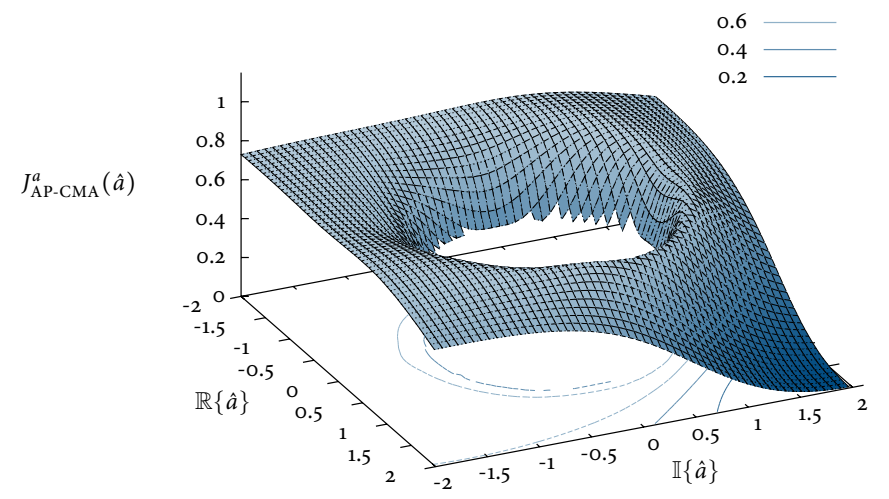

FiguRE 5.3 - Error-performance surface $J_{\mathrm{AP}-\mathrm{CMA}}^{2+2 j}(\hat{a})(\mathrm{M}=9)$.

is used for compensating the channel's response. Note that, values of $|\hat{a}|<1$ are not shown because these result in an unbounded equalizer impulse response. By choosing appropriate initial conditions for $\hat{a}$, such as $\hat{a}[1]=2+2 j$, the gradientdescent optimization would never converge to such an unbounded response.

The error-performance surface of our CM-based nonlinearity is multimodal. However, no other zero-memory nonlinear cost function is known, which would always result in global convergence [26]. The basin of attraction to a global minimum becomes larger if the zero of the nonminimum-phase channel moves further away from the unit circle. The latter can be seen in figure 5.3, wherein $a$ is set to $2+2 j$. Effectively, in such a scenario the chance of misconvergence decreases.

\subsection{The AP-CMA cost Minimizer}

To derive the gradient-descent minimizer of the all-pass CMA, Wirtinger calculus is used. Section 4.4 shortly elaborated on Wirtinger calculus. However, a detailed discussion of this calculus is given in section 5.4.1. The stochastic gradient-descent minimizer of the all-pass CMA is derived in section 5.4.2.

\subsubsection{WIRTINGER CALCULUS}

As most cost functions, our AP-CMA cost function is real-valued $\left(J_{\mathrm{AP}-\mathrm{CMA}}: \mathbb{C} \rightarrow\right.$ $\mathbb{R})$. Complex-valued cost functions would not be useful, because ordering is not defined in the complex domain. For minimization of $J_{\mathrm{AP}-\mathrm{CMA}}$, we use a stochastic gradient-descent method. Unfortunately, the function $J_{\mathrm{AP}-\mathrm{CMA}}$ is not complex differentiable, since it does not satisfy the Cauchy-Riemann equations. This problem of complex differentiability occurs in many other signal processing methods. 
An elegant approach to relax complex differentiability is to work in the Wirtinger calculus. The latter provides a means to overcome the hassle of decomposing the complex cost function into real and imaginary parts and differentiating both parts separately. In the Wirtinger calculus the cost function is decomposed into two complex conjugate variables and these variables are treated as if they are independent from each other. The work by Brandwood [9] states:

Theorem.[Wirtinger Calculus] Let $f: \mathbb{C}^{N} \rightarrow \mathbb{R}$ be a real-valued scalar function of a complex vector $\mathbf{z}$. Let $f(\mathbf{z})=g\left(\mathbf{z}, \mathbf{z}^{*}\right)$, where $g: \mathbb{C}^{N} \times \mathbb{C}^{N} \rightarrow \mathbb{R}$ is a real-valued function of two complex variables and $g$ is analytic with respect to each element in $\mathbf{z}$ and $\mathbf{z}^{*}$. Then either of the conditions $\nabla_{\mathbf{z}} g=0$ or $\nabla_{\mathbf{z}^{*}} g=0$ is necessary and sufficient to determine a stationary point of $f$. The partial gradient of $g$ with respect to $\mathbf{z}^{*}\left(\nabla_{\mathbf{z}^{*}} g\right)$, rather than the partial gradient of $g$ with respect to $\mathbf{z}\left(\nabla_{\mathbf{z}} g\right)$, gives the direction of the maximum rate of change of $f$ with respect to $\mathbf{z}$.

From the above mathematical context, we infer that for a gradient-descent optimization of $f$, the gradient $\nabla_{\mathbf{z}^{*}} g$ is preferred over the gradient $\nabla_{\mathbf{z}} g$. Brandwood also notes that the gradient $\nabla_{\mathbf{z}^{*}} g$ typically leads to neater mathematical expressions [9]. Therefore, in our derivation, we always derive the partial gradient with respect to the complex conjugate vector.

\subsubsection{MINIMIZER DERIVATION}

Below the instantaneous gradient-descent minimizer of the AP-CMA is shown, followed by a step-by-step derivation based on Wirtinger calculus.

Corollary.[The AP-CMA minimizer]

An estimate for the channel's nonminimum-phase zero $\hat{a}$ can be found using the following gradient-descent minimization:

$$
\begin{aligned}
\hat{a}[k+1] & =\hat{a}[k]-\mu \cdot\left[\mid\left(\left.\mathbf{w}(\hat{a})^{T} \mathbf{x}\right|^{2}-1\right] .\right. \\
& \left\{\mathbf{w}_{1}(\hat{a}) \mathbf{x}^{*} \mathbf{x}^{T} \mathbf{w}(\hat{a})+\mathbf{w}(\hat{a})^{H} \mathbf{x}^{*} \mathbf{x}^{T} \mathbf{w}_{2}(\hat{a})\right\},
\end{aligned}
$$

where,

$$
\begin{aligned}
& \mathbf{w}_{1}(\hat{a})=\left|\begin{array}{c}
w_{1}(-M+1, \hat{a}) \\
\vdots \\
w_{1}(0, \hat{a})
\end{array}\right| \quad \text { with } w_{1}(m, \hat{a})=-\hat{a} m\left(\hat{a}^{*}\right)^{m-1}+(m-1)\left(\hat{a}^{*}\right)^{m-2}, \\
& \mathbf{w}_{2}(\hat{a})=\left|\begin{array}{c}
w_{2}(-M+1, \hat{a}) \\
\vdots \\
w_{2}(0, \hat{a})
\end{array}\right| \quad \text { with } w_{2}(m, \hat{a})=-\hat{a}^{m} u[-m-1] .
\end{aligned}
$$

Herein, $\mathbf{x}$ are the equalizer input samples, $\mathbf{w}$ are the equalizer weights, $\mu$ is the convergence factor and $M$ is the order of the blind equalizer. 
Proof. The weight vector $\mathbf{w}(\hat{a})$ for an all-pass equalizer, where $\hat{a}$ is the zero to be compensated, was given in eq. 5.5. First, we rewrite $J_{\mathrm{AP}-\mathrm{CMA}}(\hat{a})$ in terms of $\hat{a}$ and $\hat{a}^{*}$ by regarding this weight vector $\mathbf{w}(\hat{a})$ as a function of $\hat{a}$ and $\hat{a}^{*}$ :

$$
J_{\mathrm{AP}-\mathrm{CMA}}(\hat{a})=G\left(\hat{a}, \hat{a}^{*}\right)=E\left\{\left[\mid\left(\left.\mathbf{w}\left(\hat{a}, \hat{a}^{*}\right)^{T} \mathbf{x}\right|^{2}-1\right]^{2}\right\} .\right.
$$

Note that, function $G\left(\hat{a}, \hat{a}^{*}\right)$ is analytic with respect to $\hat{a}$ and $\hat{a}^{*}$. Therefore, according to the Wirtinger calculus in section 5.4.1, we may partially differentiate $G\left(\hat{a}, \hat{a}^{*}\right)$ with respect to $\hat{a}^{*}$ to find the direction of the maximum rate of change of $J_{\mathrm{AP}-\mathrm{CMA}}$ with respect to $\hat{a}$.

Start partial differentiation by expanding $G\left(\hat{a}, \hat{a}^{*}\right)$ using the chain rule:

$$
\frac{\delta G\left(\hat{a}, \hat{a}^{*}\right)}{\delta \hat{a}^{*}}=2 E\left\{\left[\left|\mathbf{w}\left(\hat{a}, \hat{a}^{*}\right)^{T} \mathbf{x}\right|^{2}-1\right] \frac{\delta}{\delta \hat{a}^{*}}\left\{\mathbf{w}\left(\hat{a}, \hat{a}^{*}\right)^{H} \mathbf{x}^{*} \mathbf{x}^{T} \mathbf{w}\left(\hat{a}, \hat{a}^{*}\right)\right\}\right\} .
$$

Thereafter, the partial derivative of the second part is found by application of the product rule:

$$
\begin{gathered}
\frac{\delta G\left(\hat{a}, \hat{a}^{*}\right)}{\delta \hat{a}^{*}}=2 E\left\{\left[\left|\mathbf{w}\left(\hat{a}, \hat{a}^{*}\right)^{T} \mathbf{x}\right|^{2}-1\right]\left(\frac{\delta}{\delta \hat{a}^{*}} \mathbf{w}\left(\hat{a}, \hat{a}^{*}\right)^{H}\right) \mathbf{x}^{*} \mathbf{x}^{T} \mathbf{w}\left(\hat{a}, \hat{a}^{*}\right)+\right. \\
\mathbf{w}\left(\hat{a}, \hat{a}^{*}\right)^{H} \mathbf{x}^{*} \mathbf{x}^{T} \frac{\delta}{\delta \hat{a}^{*}} \mathbf{w}\left(\hat{a}, \hat{a}^{*}\right),
\end{gathered}
$$

having,

$$
\begin{array}{ll}
\frac{\delta}{\delta \hat{a}^{*}} \mathbf{w}\left(\hat{a}, \hat{a}^{*}\right)^{H}=-a m\left(a^{*}\right)^{m-1}+(m-1)\left(a^{*}\right)^{m-2}, & \\
\frac{\delta}{\delta \hat{a}^{*}} \mathbf{w}\left(\hat{a}, \hat{a}^{*}\right)=-a^{m} u[-m-1], & m=(-M+1), \ldots, 0 .
\end{array}
$$

To find an estimate for $\hat{a}$, the derivative $\frac{\delta}{\delta \hat{a}^{*}} G\left(\hat{a}, \hat{a}^{*}\right)$ can now be used in a gradientdescent as follows:

$$
\hat{a}[k+1]=\hat{a}[k]-\mu \cdot \frac{\delta}{\delta \hat{a}^{*}} G\left(\hat{a}, \hat{a}^{*}\right)
$$

By absorbing the factor 2 in the convergence factor $\mu$ and dropping the expected value operator, we find the instantaneous gradient-descent minimizer shown in eq. 5.9. 
To evaluate the AP-CMA, we illustrate its convergence behaviour in section 5.5.1. Thereafter, the AP-CMA is compared to the conventional CMA in terms of ISI in section 5.5.2.

For simulations, the following real single-pole single-zero all-pass channel from literature is used [33]:

$$
H(z)=\frac{0.7-z^{-1}}{1-0.7 z^{-1}}
$$

In the following simulations, both the all-pass channel transfer $H(z)$ and the equalizer are implemented using an eight-tap FIR filter.

In [33], channel $H(z)$ also includes a constant phase offset. This phase offset has been removed, to facilitate analysis, because the CMA and the AP-CMA do not correct rotations of the symbol constellation. Therefore, other means are necessary to perform derotation of the constellation, e.g., the derotator of the QPSK demodulator or a PLL [95].

The convergence factor of the CMA is set to $5 \cdot 10^{-3}$. A factor in this order of magnitude typically results in decent equalization performance. To initialize the CMA, the fourth tap of the weight vector $\mathbf{w}$ is set to one and all others to zero. Initialization of the CMA weight vector was discussed in section 4.4 .

The convergence factor of the AP-CMA is set to $1.5 \cdot 10^{-1}$. Choosing an initial estimate $\hat{a}[1]$ for the zero position is discussed in section 5.5.1.

\subsubsection{CONVERGENCE BEHAVIOUR ANALYSIS}

The error-performance surface of the AP-CMA can be visualized in a contour plot. In such a plot, the gradient-descent paths of the AP-CMA can be included to study the algorithm's convergence behaviour. This relatively simple representation of the search space is helpful to make informed decisions on, e.g., initial conditions for the AP-CMA.

As an example, the descent paths of our algorithm for the channel $H(z)$ and two initial values of the zero position $(\hat{a}[1]=5+0 j$ and $\hat{a}[1]=2+2 j)$ are shown in figure 5.4.

The values of $\hat{a}[1]$, being used in the simulations of figure 5.4, have equal costs. Clearly, both gradient-descent paths find the correct zero position $\left(\frac{1}{0.7}+0 j\right)$. However, in the contour plot we recognize a steeper gradient in the imaginary direction than in the real direction. Therefore, one can conclude that, for channels having real zeros, our adaptive algorithm can possibly benefit from having a non-zero imaginary component in the initial estimate.

Although not shown in figure 5.4, simulations with $16 \mathrm{~dB}$ AWGN still result in correct convergence. However, to prevent ill-convergence, the convergence factor $\mu$ should be chosen smaller in the case of larger additive noise levels. 


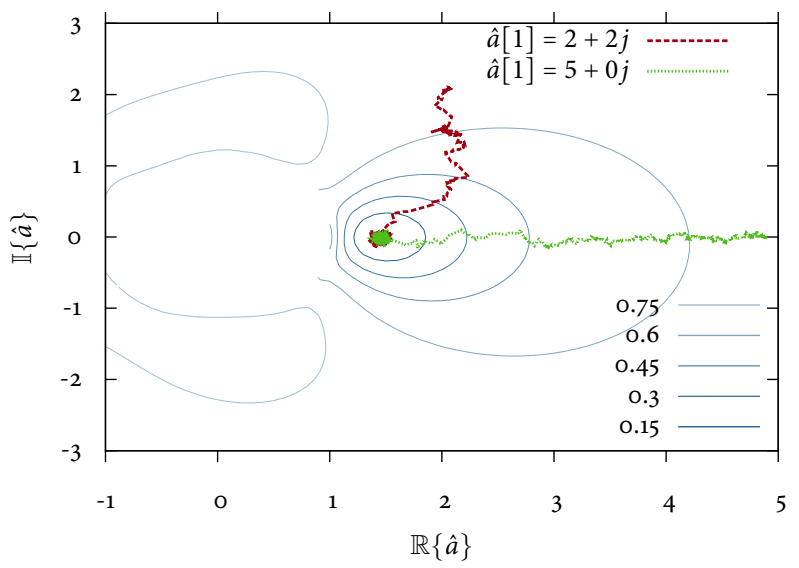

FIGURE 5.4 - Contour plot of $J_{\mathrm{AP}-\mathrm{CMA}}^{\frac{1}{0.7}+0 j}(\hat{a})$ annoted with the gradient-descent estimates $\hat{a}[n]$ of the AP-CMA for two different starting positions.

\subsubsection{EQUALIZATION PERFORMANCE COMPARISON}

The equalization performance of the AP-CMA is studied in terms of the ISI level of the aggregate channel. For the aggregate channel response $\mathbf{p}=\mathbf{h} * \mathbf{w}(\hat{a})$, a numerical measure of the ISI is found using [52]:

$$
\text { ISI }=\frac{\sum|p[n]|^{2}-\left|p\left[n_{\max }\right]\right|^{2}}{\left|p\left[n_{\max }\right]\right|^{2}} .
$$

Herein, $n_{\max }$ is the index of the impulse response component having the largest magnitude. Clearly, if the impulse component with the largest power $\left(\left|p\left[n_{\max }\right]\right|^{2}\right)$ is equal to the sum of all powers $\left(\sum|p[n]|^{2}\right)$ then ISI is minimal. Note that $*$ represents discrete-time convolution.

The ISI levels of the CMA and the AP-CMA, during adaptive equalization of channel $H(z)$, are shown in figure 5.5. Based on the ISI levels after convergence, we recognize a more accurate compensation of the nonminimum-phase zero by the AP-CMA compared to the conventional CMA. For eight-tap AP-CMA equalizers, the ISI levels after convergence are approximately $10 \mathrm{~dB}$ smaller. Such an ISI reduction enables the use of higher-order PSK modulation schemes and is advantageous for channels corrupted by high levels of AWGN.

In figure 5.5, the CMA equalizer and the two instances of the AP-CMA equalizer have similar ISI levels for the initial aggregate channel response. The AP-CMA typically provides comparable or faster convergence than the conventional CMA. 


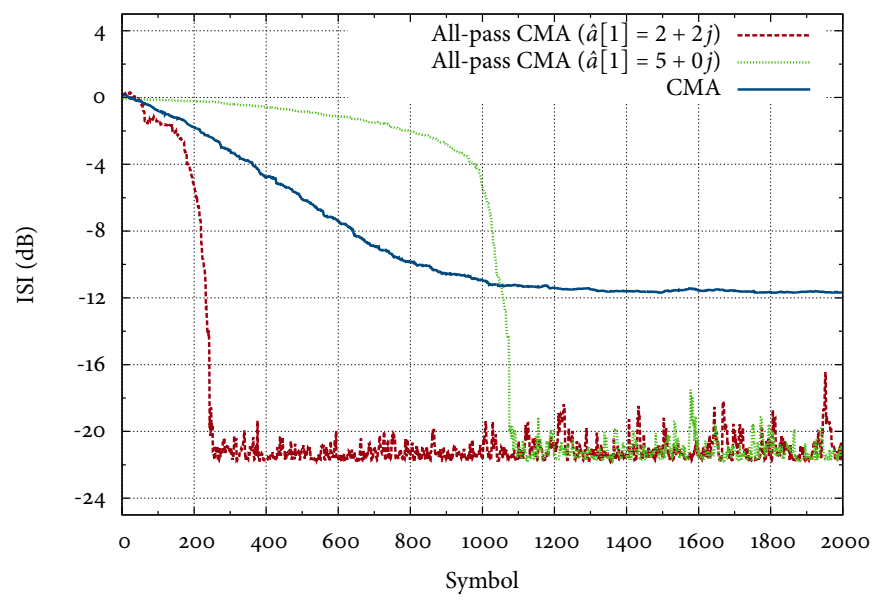

FIGURE 5.5 - Aggregate channel ISI levels for CMA and all-pass CMA.

Nevertheless, the convergence speed of both algorithms strongly depends on their initial weights and the convergence factor $\mu$. As explained in section 5.5.1, being able to visualize to search space of the AP-CMA, can be valuable to find appropriate initial weights. A theoretical analysis of the convergence speed of the AP-CMA is beyond the scope of this thesis.

\subsection{CONCLUSIONS AND FUTURE WORK}

A nonminimum-phase channel can always be decomposed into a cascade of (i) a minimum-phase response and (ii) an all-pass response. In general, blind equalization of such an all-pass response is more difficult than blind equalization of the minimum-phase response, since HOS are required to estimate the nonminimumphase zero positions of the all-pass response. In our blind equalization method, the search space of our equalizer is reduced to merely all-pass responses to provide faster and more accurate compensation of the all-pass part of a nonminimum-phase channel.

Our dimensionality reduction has been performed by parameterizing the CMA cost function in terms of the nonminimum-phase zero location of the all-pass response to be compensated. Effectively, this led to a search space reduction from $M$ complex weights to a single complex weight $\left(\mathbb{C}^{M} \rightarrow \mathbb{C}\right)$.

The AP-CMA can only be used for compensation of a single-pole single-zero allpass part of a nonminimum-phase channel. However, multiple AP-CMA equalizers could be used in a filterbank to enable compensation of multiple nonminimumphase zeros. The effectiveness of such a filterbank, for more realistic underwater channel responses, should be investigated. 
Equalization performance and convergence behaviour of the AP-CMA have been compared to the CMA. The AP-CMA provides a far more accurate compensation of the all-pass response, while having a similar or improved convergence rate. E.g., the ISI floor of an eight-tap AP-CMA equalizer is $10 \mathrm{~dB}$ lower than for the conventional CMA.

The error-performance surface of the AP-CMA can be visualized in a surface plot. For more complex channels, it would be of interest to perform an extensive analysis of the effect of choosing certain initial conditions. 



\section{CONCLUSIONS AND FUTURE WORK}

\subsection{BLIND EQUALIZATION FOR UNDERWATER COMMUNICATIONS}

To support the growing interest in underwater monitoring, large-scale underwateracoustic sensor networks (UW-ASNs) can be employed. Communication between nodes in the harsh underwater environment poses significant challenges. In this thesis, adaptive spatial and spectral equalization methods were presented to compensate for the time-varying distortion caused by the underwater acoustic channel.

In general, the communication capacity of the underwater channel is low and underwater acoustic nodes have limited energy resources. To reduce energy consumption, structural properties of the transmitted signal (instead of training sequences) were exploited to calculate equalizer weight adjustments. The latter is known as blind equalization. To optimize spectral efficiency, while being power-efficient, our underwater transmissions are QPSK modulated. QPSK transmissions have a constant modulus. The blind equalization methods that were discussed in this thesis exploit deviations of the equalizer output from a constant modulus as a training sequence substitute. A well-known blind equalization method that uses this constant modulus property is called the constant modulus algorithm (CMA).

Since no standard underwater channel model exists, real-life experiments are necessary for proper evaluation. Existing systems for underwater acoustic signal processing did not provide enough flexibility for our experiments. Therefore, a flexible multi-channel underwater testbed has been developed and used in our experiments.

For mitigation of signal distortion in the spatial dimension, various blind spatial equalization methods have been discussed. First, the CMA has been applied to empirical underwater data. In our experiment, CMA's behavior reveals asymptotic convergence to the optimum equalizer weights. Thereafter, a stricter blind method, known as the extended CMA (E-CMA), has been studied in a spatial underwater context. The E-CMA is stricter since it exploits both the constant modulus and the phase distribution of QPSK signals. For the same dataset, the MSE level of the ECMA converges faster compared to the CMA. Also, the angular CMA (A-CMA) has been presented. In contrast to other blind methods, the A-CMA calculates steering angle updates instead of weight vector updates. This approach is attractive in mixedsignal hierarchical arrays where distribution of the steering angle is needed. The 
applicability of the A-CMA for underwater communications should further be investigated, since the algorithm was originally designed for tracking a strong LoS arrival in an environment with practically no multipath.

In the underwater environment, multipath components add up constructively and destructively producing frequency-selective channel distortion. To compensate for frequency-selectivity, blind spectral equalization has been studied. A directpath underwater transmission might arrive later than its reflections. The latter is known as nonminimum-phase behavior. A blind method, named the all-pass CMA (AP-CMA), was introduced for faster and more accurate compensation of nonminimum-phase channel distortion.

\subsubsection{CONTRIBUTIONS}

In the context of blind equalization for underwater communications, as well as in a more general context, the contributions of this thesis are:

"An assessment of the quantitative characterization of the underwater channel that elaborates on the channel's properties relevant for the design of underwater communication hardware and physical layer algorithms (chapter 2) [KCH:4] .

"A multi-channel underwater testbed developed to evaluate physical layer algorithms, and used in localization and spatial equalization experiments (chapters 3 and 4 ) [KCH:4, 5] .

»A blind spatial equalization algorithm, designated the extended CMA (ECMA), that provides tracking of the signal-of-interest, while simultaneously correcting phase offsets (chapter 4 ) $[\mathrm{KCH}: 1]$.

» A blind spatial equalization algorithm, called the angular CMA (A-CMA), that mitigates distortion and simultaneously provides a means for steering angle distribution in mixed-signal hierarchical arrays (chapter 4) [ $\mathrm{KCH}: 2]$.

» A blind spectral equalization algorithm, called the all-pass CMA (AP-CMA), that provides a fast and accurate compensation for the distortion caused by single-pole single-zero nonminimum-phase channels (chapter 5) $[\mathrm{KCH}: 3]$.

\subsubsection{RECOMMENDATIONS}

Recommendations for further analysis and improvement of the blind methods presented in this thesis are given below.

The CMA and the E-CMA have been evaluated based on empirical data. Evidently, the MSE levels of the E-CMA converge faster than those of the CMA. However, these measurements were acquired in the reverberant, but relatively time-invariant environment of the dive-center water tank. Further theoretical and empirical studies should elaborate on the error performance and the convergence behaviour of the E-CMA, in more dynamic environments. 
The dimensionality reduction of the A-CMA, from $N$ complex weights to a single steering angle, might be overly restrictive. For future work, we suggest to moderate restrictions by incorporating an additional parameter into the optimization. E.g., apart from a linear phase taper expressed in terms of the steering angle, it is interesting to investigate the inclusion of an amplitude taper which is expressed in terms of a single parameter. Additional freedom might probably improve the MSE performance, while still providing faster convergence than the conventional CMA. In general terms, expressing the CMA cost function in a single or multiple parameters could be designated as the parameterized CMA.

The convergence region of the A-CMA is limited. An increase of the number of array elements decreases the range where global convergence can be guaranteed. By applying other optimization methods, it might be possible to improve convergence, independent of (i) the number of array elements and (ii) the initial steering angle.

The AP-CMA can only compensate for a single-pole single-zero channel. For channels affected by more than a single nonminimum-phase zero, a filterbank with multiple AP-CMA equalizers would be effective. Ideally, an AP-CMA equalizer is developed that can compensate a specified (maximum) number of nonminimumphase zeros.

Altogether, we have discussed blind adaptive equalization as an energy-efficient means to provide fast and accurate compensation of underwater channel distortion. Application of our proposed methods, together with the recommendations, seems a promising approach for future underwater communications. 



\section{IDENTIFIABILITY OF NONMINIMUM-PHASE CHANNELS}

\section{A.1 THEOREMS}

In chapter 5, we identified the nonminimum-phase channel response by implicitly using HOS by means of the nonlinearity of the A-CMA cost criterion. This appendix shortly elaborates on two major theorems which define the relationship between explicit HOS and ideal blind equalization of nonminimum-phase channels.

\section{A.1.1 BENVENISTE-GOURSAT-RUGET}

The Benveniste-Goursat-Ruget [6] theorem relates to the scenario where the nonGaussian i.i.d. samples $s[k]$ are observed through a noiseless LTI with impulse response $h[m]$. An estimate of the source $y[k]=\hat{s}[k]$ must be obtained (using an equalizer) given merely the input samples $x[k]$ and knowledge of the PDF of $s[k]$. The Benveniste-Goursat-Ruget theorem states that, if the PDFs of $s[k]$ and $y[k]=\hat{s}[k]$ are identical, then correct estimation is guaranteed, except for a possible complex gain factor and delay as in eq. 5.1.

\section{A.1.2 SHALVI-WEINSTEIN}

Shalvi and Weinstein [71] proof that a necessary and sufficient condition for perfect equalization only requires equalization of a few moments of the probability distributions of the in- and output signals. Therefore, with respect to BenvenisteGoursat-Ruget's theorem, the theorem of Shalvi and Weinstein is less stringent.

Shalvi and Weinstein use the Cauchy-Schwarz inequality to connect higher-order statistics to the ideal equalization criterion given in Equation eq. 5.1. An overview of this proof of Shalvi and Weinstein [71] can be seen below.

Lemma $1 E\left\{|y[k]|^{2}\right\}=E\left\{|s[k]|^{2}\right\} \sum_{m}|p[m]|^{2}$

Proof. Start by writing output $y[k]$ as the convolution of the non-Gaussian i.i.d. (zero-mean) input samples $s[k]$ with the compound channel response $p[m]$ :

$$
y[k]=s[k] *(h[m] * w[m])=\sum_{m} s[k-m] p[m]
$$


Herein, * represents discrete-time convolution. The magnitude squared of $y[k]$ can be written as:

$$
\begin{aligned}
E\left\{|y[k]|^{2}\right\} & =E\left\{\left|\sum_{m} s[k-m] p[m]\right|^{2}\right. \\
& =E\left\{\sum_{m} s[k-m] p[m] \sum_{l} s[k-l]^{*} p[l]^{*}\right\} \\
& =\sum_{m} \sum_{l} E\left\{s[k-m] s[k-l]^{*}\right\} p[m] p[l]^{*}
\end{aligned}
$$

Due to values of $s[k]$ being mutually independent, iff $m=l$ then $\sum_{m} \sum_{l} E\left\{s[k-m] s[k-l]^{*}\right\} \neq 0$. Therefore, $E\left\{|y[k]|^{2}\right\}$ can be rewritten as:

$$
\begin{aligned}
E\left\{|y[k]|^{2}\right\} & =\sum_{m} E\left\{s[k-m] s[k-m]^{*}\right\} p[m] p[m]^{*} \\
& =\sum_{m} E\left\{s[k-m] s[k-m]^{*}\right\}|p[m]|^{2}
\end{aligned}
$$

The variance $E\left\{s[k-m] s[k-m]^{*}\right\}$ of $s[k]$ is time-invariant because of its identical distribution property. Therefore, we can write:

$$
E\left\{|y[k]|^{2}\right\}=E\left\{|s[k]|^{2}\right\} \sum_{m}|p[m]|^{2}
$$

Lemma $2 \mathrm{~K}(y[k])=\mathrm{K}(s[k]) \sum_{m}|p[m]|^{4}$

Proof. Analog to eq. A.2-eq. A.7, an estimate of the fourth power of the equalizer magnitude $E\left\{|y[k]|^{4}\right\}$ can also be rewritten in terms of $s[k]$ and $p[k]$ :

$$
\begin{aligned}
E\left\{|y[k]|^{4}\right\} & \left.=\left.\left(E\left\{|s[k]|^{4}\right\}-2 E^{2}\left\{|s[k]|^{2}\right\}-\mid E\{s[k]]^{2}\right\}\right|^{2}\right) \cdot \sum_{m}|p[m]|^{4} \ldots \\
& +2 E^{2}\left\{|s[k]|^{2}\right\} \cdot\left(\sum_{m}|p[m]|^{2}\right)^{2}+\left|E\left\{s[k]^{2}\right\}\right|^{2} \cdot\left|\sum_{m} p[m]^{2}\right|^{2}
\end{aligned}
$$

An estimate of the complex kurtosis $\mathrm{K}(y[k])$ of $y[k]$ can found using:

$$
\mathrm{K}(y[k]) \triangleq E\left\{|y[k]|^{4}\right\}-2 E^{2}\left\{|y[k]|^{2}\right\}-\left|E\left\{y[k]^{2}\right\}\right|^{2}
$$

Now assume the lemma is true. By expanding the kurtosis terms, using eq. A.9, lemma 2 can be given as:

$$
\begin{aligned}
\left.E\left\{|y[k]|^{4}\right\}-2 E^{2}\left\{|y[k]|^{2}\right\}-\mid E\{y[k]]^{2}\right\}\left.\right|^{2} & =\left(E\left\{|s[k]|^{4}\right\}-2 E^{2}\left\{|s[k]|^{2}\right\}-\left|E\left\{s[k]^{2}\right\}\right|^{2}\right) \sum_{m}|p[m]|^{4} \\
E\left\{|y[k]|^{4}\right\} & =\left(E\left\{|s[k]|^{4}\right\}-2 E^{2}\left\{|s[k]|^{2}\right\}-\left|E\left\{s[k]^{2}\right\}\right|^{2}\right) \sum_{m}|p[m]|^{4} \\
& +2 E^{2}\left\{|y[k]|^{2}\right\}+\left|E\left\{y[k]^{2}\right\}\right|^{2}
\end{aligned}
$$


The lemma is now shown to be correct by demonstrating equality between the last two terms of Equation A.8 and the last two terms of Equation A.10. The latter is done by applying lemma 1 and $E\left\{y[k]^{2}\right\}=E\left\{s[k]^{2}\right\} \sum_{m} p[m]^{2}$ :

$$
\begin{aligned}
& 2 E^{2}\left\{|y[k]|^{2}\right\}=2 \cdot\left(E\left\{|s[k]|^{2}\right\} \sum_{m}|p[m]|^{2}\right)^{2}=2 E^{2}\left\{|s[k]|^{2}\right\} \cdot\left(\sum_{m}|p[m]|^{2}\right)^{2} \\
& \left|E\left\{y[k]^{2}\right\}\right|^{2}=\left|E\left\{s[k]^{2}\right\} \sum_{m} p[m]^{2}\right|^{2}=\left|E\left\{s[k]^{2}\right\}\right|^{2} \cdot\left|\sum_{m} p[m]^{2}\right|^{2}
\end{aligned}
$$

Lemma 1 and 2 are the basis of the Shalvi-Weinstein equalization theorem.

Corollary. $\sum_{m}|p[m]|^{4} \leqslant\left(\sum_{m}|p[m]|^{2}\right)^{2}$

The corollary is related to the Cauchy-Schwarz inequality and is stated in [71].

Theorem.[Shalvi-Weinstein Equalization Theorem]

If $E\left\{|y[k]|^{2}\right\}=E\left\{|s[k]|^{2}\right\}$ then:

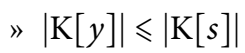

» $|\mathrm{K}[y]|=|\mathrm{K}[s]|$ iff $p[m]$ has the form of $p_{\mathrm{unit}[m]}{ }^{1}$.

Essentially, the Shalvi-Weinstein theorem states that a necessary and sufficient condition for equalization is 'power normalization' $\left(E\left\{|y[k]|^{2}\right\}=E\left\{|s[k]|^{2}\right\}\right)$ and kurtosis matching $(|\mathrm{K}[y]|=|\mathrm{K}[s]|)$.

Proof. Recapitulate the corollary and lemma 1 and 2:

$$
\begin{aligned}
& » \sum_{m}|p[m]|^{4} \leqslant\left(\sum_{m}|p[m]|^{2}\right)^{2}
\end{aligned}
$$

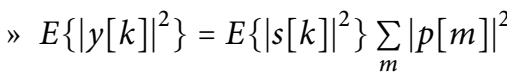

$$
\begin{aligned}
& \text { " } \mathrm{K}(y[k])=\mathrm{K}(s[k]) \sum_{m}|p[m]|^{4}
\end{aligned}
$$

If and only if $E\left\{|y[k]|^{2}\right\}=E\left\{|s[k]|^{2}\right\}$ then $\left(\sum_{m}|p[m]|^{2}\right)^{2}=1$. Now based on the corollary $\sum_{m}|p[m]|^{4} \leqslant 1$. In this case, $|\mathrm{K}[y]| \leqslant|\mathrm{K}[s]|$.

Equality $|\mathrm{K}[y]|=|\mathrm{K}[s]|$ holds iff $\sum_{m}|p[m]|^{4}=\left(\sum_{m}|p[m]|^{2}\right)^{2}=1$. The latter requires at most one nonzero (unit magnitude) element in the $p$ vector, which corresponds to the ideal equalization criterion $p_{\text {unit }[m]}$.

\footnotetext{
${ }^{1} p_{\text {unit }[m]}$ is a filter allowed to impose a delay $d_{\mathrm{eq}}$ and a phase shift $\theta: p_{\mathrm{unit}}[m]=\delta_{k}\left[m-d_{\mathrm{eq}}\right] \cdot e^{j \theta}$.
} 



\section{ACronyms}

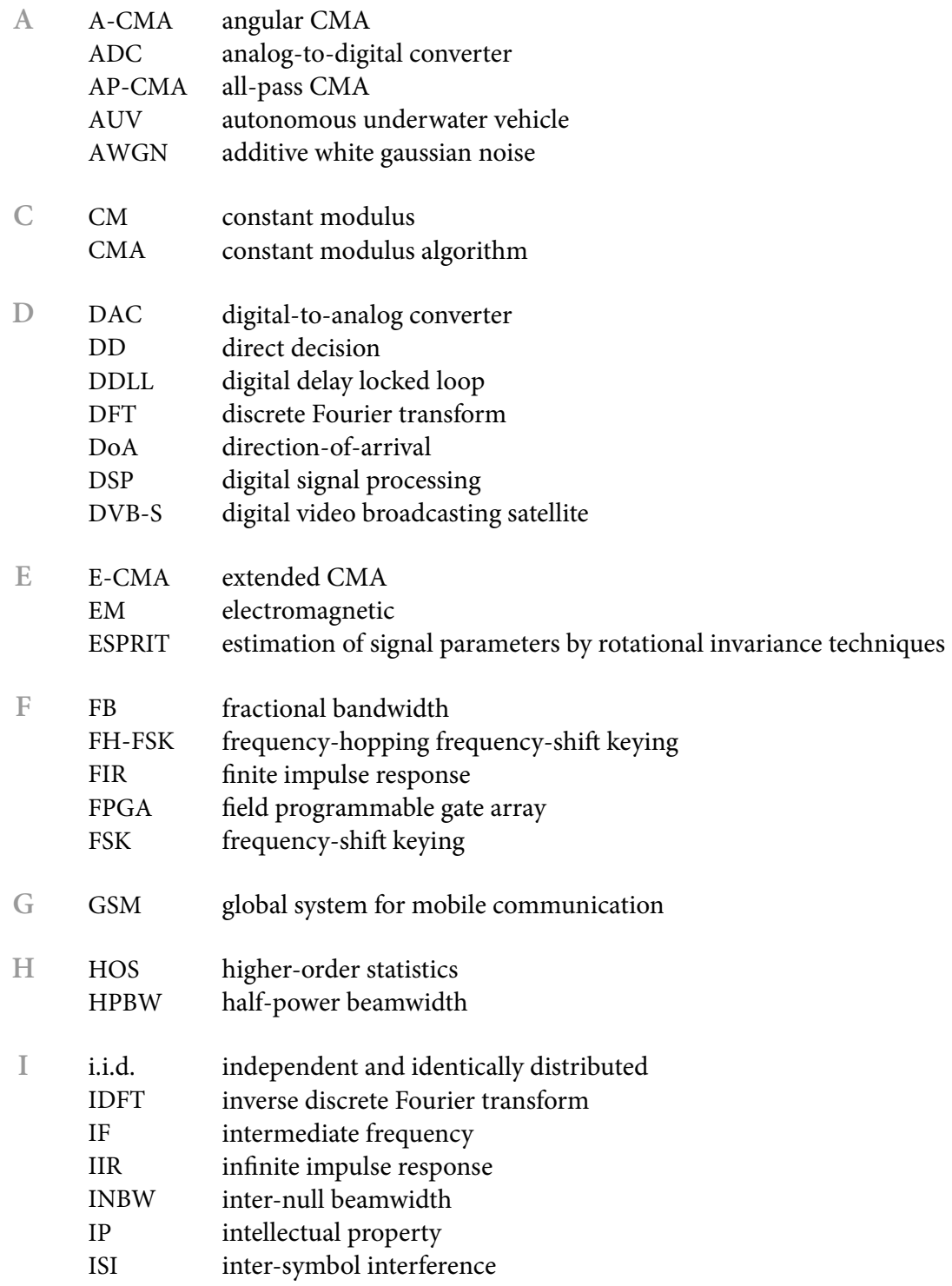




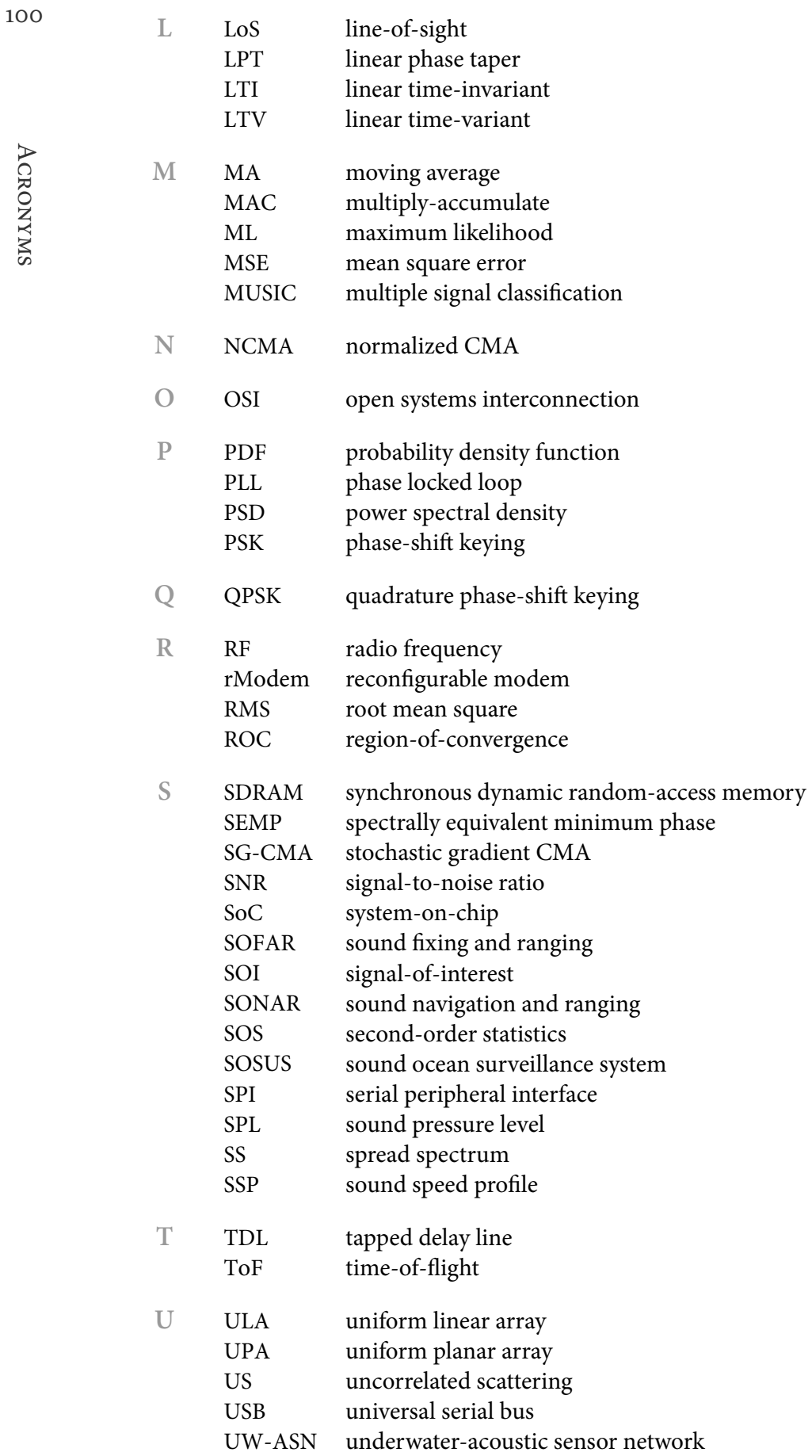


W WSS wide-sense stationary

WSSUS wide-sense stationary uncorrelated scattering 



\section{Nomenclature}

$\begin{array}{ll}\gamma_{r \mathbf{x}} & \text { Cross-covariance vector } \\ \boldsymbol{\tau} & \text { Time delay vector } \\ \boldsymbol{R}_{\mathrm{xx}} & \text { Spatial autocovariance matrix } \\ \delta_{k} & \text { Kronecker delta function } \\ \gamma_{h h} & \text { Autocorrelation function of } h \\ \hat{a} & \text { Estimate for the channel's nonminimum-phase zero } \\ \lambda & \text { Wavelength (m) } \\ \mathbf{a} & \text { Wave propagation direction } \\ \mathbf{h}(a) & \text { All-pass channel having a zero at } a \\ \mathbf{p}_{n} & \text { Cartesian position vector of } n^{\text {th }} \text { array element } \\ \mathbf{u} & \text { Far-field source direction vector } \\ \mathbf{w}(a) & \text { All-pass channel equalizer for channel with a zero at } a \\ \mathbf{x}(t) & \text { Continuous-time array element sample vector } \\ \mathbf{x}[k] & \text { Discrete-time array element sample vector } \\ \mu & \text { Convergence factor } \\ v_{p} & \text { Doppler shift of } p^{\text {th }} \text { propagation path } \\ \phi & \text { Azimuth angle } \\ \tau_{p} & \text { Time delay of } p^{\text {th }} \text { propagation path } \\ \mathbf{d} & \text { Array response vector } \\ \mathbf{P} & \text { Array elements position matrix } \\ \mathbf{v} & \text { Array manifold vector } \\ \mathbf{w} & \text { Equalizer weight vector } \\ \mathbf{w}_{\mathrm{opt}} & \text { Wiener-Hopf optimum } \\ \theta & \text { Polar angle } \\ \theta_{0} & \text { Steering angle } \\ & \end{array}$




\begin{tabular}{|c|c|c|}
\hline \multirow{3}{*}{104} & $\theta_{g}$ & (Complete) grating lobe angle \\
\hline & $\varepsilon$ & Transmission loss offset \\
\hline & $a$ & Zero location of channel \\
\hline \multirow{5}{*}{ 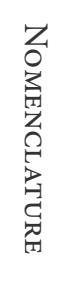 } & $a(f)$ & Absorption coefficient \\
\hline & $B$ & Beamformer response \\
\hline & $b$ & Pole location of channel \\
\hline & $c$ & Underwater speed of sound $\left(\mathrm{m} \mathrm{s}^{-1}\right)$ \\
\hline & $c_{\mathrm{eq}}$ & Equalizer complex gain factor \\
\hline & $D$ & Array aperture \\
\hline & $d$ & Array spacing (m) \\
\hline & $d_{\mathrm{eq}}$ & Equalizer delay \\
\hline & $f$ & Frequency \\
\hline & $f_{h}$ & Highest frequency in signal-of-interest \\
\hline & $f_{l}$ & Lowest frequency in signal-of-interest \\
\hline & $f_{r}$ & Reference frequency $(=1 \mathrm{~Hz})$ \\
\hline & $h(\tau)$ & LTI impulse response \\
\hline & $h(t, \tau)$ & LTV impulse response \\
\hline & $H(z)$ & Channel $z$-domain characterization \\
\hline & $H_{\mathrm{AP}}^{a}(z)$ & All-pass part of nonminimum-phase channel \\
\hline & $H_{\mathrm{NMP}}(z)$ & Nonminimum-phase channel \\
\hline & $h_{p}$ & Complex gain factor of $p^{\text {th }}$ propagation path \\
\hline & $H_{\mathrm{MP}}(z)$ & Minimum-phase part of nonminimum-phase channel \\
\hline & $H(\omega)$ & LTI frequency response \\
\hline & $J_{\mathrm{AP}-\mathrm{CMA}}$ & The AP-CMA cost function \\
\hline & $J_{\mathrm{CMA}}$ & The CMA cost function \\
\hline & $J_{\mathrm{A}-\mathrm{CMA}}$ & The A-CMA cost function \\
\hline & $J_{\mathrm{E}-\mathrm{CMA}}$ & The E-CMA cost function \\
\hline & $k$ & Spreading factor \\
\hline & $k(\omega)$ & Wavenumber \\
\hline & $l_{0}$ & Reference distance (= $1 \mathrm{~m})$ \\
\hline
\end{tabular}


Number of delay taps

$N \quad$ Number of array elements

$N\left(f, s_{\mathrm{n}}, w_{\mathrm{n}}\right) \quad$ Underwater ambient noise $\left(\mu \mathrm{Pa}^{2} \mathrm{~Hz}^{-1}\right)$

$n_{0}$

Normalization factor

$N_{\mathrm{s}}\left(f, s_{\mathrm{n}}\right) \quad$ Shipping noise $\left(\mu \mathrm{Pa}^{2} \mathrm{~Hz}^{-1}\right)$

$p$

Pressure $(\mu \mathrm{Pa})$

$P(z)$

Aggregate channel $z$-domain characterization

$p_{\text {ref }}$

Reference pressure $(=1 \mu \mathrm{Pa})$

$r$

Radial distance $(\mathrm{m})$

$R_{2}$

The Godard parameter

$S$

Salinity (parts per thousand)

$s(t)$

Continuous-time source signal

$s[k]$

Discrete-time source symbols

$S_{h}(\tau, v)$

Delay-Doppler spreading function

$s_{n}$

Shipping factor

$T$

Temperature $\left({ }^{\circ} \mathrm{C}\right)$

$v[k]$

Additive white gaussian noise

$W(z)$

Equalizer $z$-domain characterization

$W_{\mathrm{AP}}^{a}(z)$

All-pass part of channel equalizer

$w_{\mathrm{AP}}^{a}[m]$

All-pass part of channel equalizer

$w_{n}$

Wind speed $\left(\mathrm{m} \mathrm{s}^{-1}\right)$

$w_{r}$

Reference wind speed $\left(=1 \mathrm{~m} \mathrm{~s}^{-1}\right)$

$W_{\mathrm{MP}}(z)$

Minimum-phase part of channel equalizer

$w_{\mathrm{MP}}[m]$

Minimum-phase part of channel equalizer

$x(t)$

Continuous-time received signal

$x[k]$

Discrete-time received signal

$y$

Equalizer output

$y[k]$

Discrete-time equalizer output

$y_{<}$

Instantaneous phase angle of $y$

$z$

Depth (m) 
$z_{0} \quad$ Reference depth $(=2 \mathrm{~m})$

$N_{\mathrm{t}}(f) \quad$ Turbulence noise $\left(\mu \mathrm{Pa}^{2} \mathrm{~Hz}^{-1}\right)$

$N_{\mathrm{w}}\left(f, w_{\mathrm{n}}\right) \quad$ Wind-related noise $\left(\mu \mathrm{Pa}^{2} \mathrm{~Hz}^{-1}\right)$

FB

Fractional bandwidth

INBW

Inter-null beamwidth

$\mathrm{NL}_{\mathrm{dB} \text { re } 1 \mu \mathrm{Pa}^{2}}(f) \quad$ Narrowband ambient noise level at the receiver

$\operatorname{SNR}_{\mathrm{dB}}(l, f) \quad$ Narrowband range- and frequency-dependent SNR

$\mathrm{SPL}_{\mathrm{dB} \text { re } 1 \mu \mathrm{Pa}} \quad$ Sound pressure level

$\mathrm{DI}_{\mathrm{dB}}(f) \quad$ Directivity of the receiver

$\mathrm{SL}_{\mathrm{dB} \text { re } 1 \mu \mathrm{Pa}} \quad$ Acoustic intensity of the source measured at $1 \mathrm{~m}$ distance

$\operatorname{TL}_{\mathrm{dB}}(l, f) \quad$ Range- and frequency-dependent transmission loss 


\section{BIBLIOGRAPHY}

[1] M. A. Ainslie. Principles of Sonar Performance Modelling. Springer-Praxis books in geophysical sciences. Springer Berlin Heidelberg, 2010. ISBN 9783540876625. URL http://books .google.nl/books?id=EqDnP-lAw40C.

[2] I. F. Akyildiz, D. Pompili, and T. Melodia. Underwater acoustic sensor networks: research challenges. Ad Hoc Networks, 3(3):257 - 279, 2005. ISSN 1570-8705. doi: DOI: 10.1016/j.adhoc.2005.01.004. URL http://www.sciencedirect.com/science/ article/B7576-4FD0HTG-1/2/401adb1308d0e999607eb9f9b041d027.

[3] B. Allen and M. Ghavami. Adaptive Array Systems, Fundamentals and Applications. John Wiley \& Sons, 2005.

[4] J. I. Antonov, D. Seidov, T. P. Boyer, R. A. Locarnini, A. V. Mishonov, H. E. Garcia, O. K. Baranova, M. M. Zweng, and D. R. Johnson. World Ocean Atlas 2009, Volume 2: Salinity. U.S. Government Printing Office, 2010. Ed. NOAA Atlas NESDIS 69.

[5] P. Bello. Characterization of randomly time-variant linear channels. Communications Systems, IEEE Transactions on, 11(4):360-393, 1963. ISSN 0096-1965. doi: 10.1109/TCOM.1963.1088793.

[6] A. Benveniste, M. Goursat, and G. Ruget. Robust identification of a nonminimum phase system: Blind adjustment of a linear equalizer in data communications. Automatic Control, IEEE Transactions on, 25(3):385-399, 1980.

[7] L. Bjørnø. Features of underwater acoustics from Aristotle to our time. Acoustical Physics, 49(1):24-30, 2003. ISSN 1063-7710. doi: 10.1134/1.1537384. URL http: //dx.doi.org/10.1134/1.1537384.

[8] R. N. Bracewell. The Fourier Transform and Its Applications. McGraw-Hill international editions. McGraw-Hill Education, 2000. ISBN 9780071160438. URL http: //books .google.nl/books?id=ecH2KgAACAAJ.

[9] D. H. Brandwood. A complex gradient operator and its application in adaptive array theory. In IEE Proceedings H (Microwaves, Optics and Antennas), volume 130, pages 11-16. IET, 1983.

[10] W. S. Burdic. Underwater Acoustic System Analysis. Peninsula Publishing, 2002.

[11] G. M. Calhoun. Third Generation Wireless Systems: Post-Shannon signal architectures. Artech House Universal Personal Communications. Artech House, 2003. ISBN 9781580535830. URL http://books.google.nl/books?id=jVte4vrh89UC. 
[12] Y. T. Chan and North Atlantic Treaty Organization. Scientific Affairs Division. Underwater Acoustic Data Processing. Applied sciences. Springer, 1989. ISBN 9780792301271. URL http://books . google.nl/books?id=tAP3zn0TtkkC.

[13] M. Chitre, S. Shahabudeen, and M. Stojanovic. Underwater acoustic communications and networking: Recent advances and future challenges. Marine Technology Society Journal, 42(1):103-116, 2008. doi: doi:10.4031/002533208786861263. URL http://www . ingentaconnect . com/content/mts/mtsj/2008/00000042/ $00000001 /$ art00014.

[14] R. F. Coates. Underwater Acoustic Systems. Wiley, New York, 1989.

[15] J.-H. Cui, J. Kong, M. Gerla, and S. Zhou. The challenges of building mobile underwater wireless networks for aquatic applications. Network, IEEE, 20(3):12-18, 2006. ISSN 0890-8044. doi: 10.1109/MNET.2006.1637927.

[16] C. Da Rocha, O. Macchi, and J. Romano. An adaptive nonlinear iir filter for selflearning equalization. In Internat. Telecom. Conf. Rio de Janeiro, Brasil, pages 6-10, 1994.

[17] O. Dahlman, H. Haak, and S. Mykkeltveit. Nuclear Test Ban: Converting Political Visions to Reality. Humanities, Social Science and Law. Springer, 2009. ISBN 9781402068850. URL http://books . google.nl/books?id=LC8BfjZTvBgC.

[18] T. Eggen, A. Baggeroer, and J. Preisig. Communication over doppler spread channels. part i: Channel and receiver presentation. Oceanic Engineering, IEEE Journal of, 25 (1):62-71, Jan 2000. ISSN 0364-9059. doi: 10.1109/48.820737.

[19] M. Ewing, G. P. Woollard, A. C. Vine, and J. L. Worzel. Recent results in submarine geophysics. Geological Society of America Bulletin, 57(10):909-934, 1946. doi: 10.1130/o016-7606(1946)57[909:RRISG]2.0.CO;2. URL http://gsabulletin . gsapubs.org/content/57/10/909. abstract.

[20] L. Freitag, M. Grund, S. Singh, J. Partan, P. Koski, and K. Ball. The WHOI micromodem: an acoustic communications and navigation system for multiple platforms. In OCEANS, 2005. Proceedings of MTS/IEEE, pages 1086 -1092 Vol. 2, sept. 2005. doi: 10.1109/OCEANS.2005.1639901.

[21] L. E. Freitag, M. Grund, J. Partan, K. Ball, S. Singh, and P. Koski. Multi-band acoustic modem for the communications and navigation aid auv. In OCEANS, 2005. Proceedings of MTS/IEEE, pages 1080-1085, Washington, DC, 2005. IEEE.

[22] W. A. Gardner, A. Napolitano, and L. Paura. Cyclostationarity: Half a century of research. Signal processing, 86(4):639-697, 2006.

[23] D. Godard. Self-recovering equalization and carrier tracking in two-dimensional data communication systems. Communications, IEEE Transactions on, 28(11):18671875, Nov. 1980. ISSN oogo-6778. doi: 10.1109/TCOM.1980.1094608.

[24] R. Gooch and J. Lundell. The CM array: An adaptive beamformer for constant modulus signals. In Acoustics, Speech, and Signal Processing, IEEE International Conference on ICASSP '86., volume 11, pages 2523-2526, Tokyo, Apr 1986. 
[25] M. Haraldsson, C. Jaspers, P. Tiselius, D. L. Aksnes, T. Andersen, J. Titelman, et al. Environmental constraints of the invasive Mnemiopsis leidyi in Scandinavian waters.

Limnol. Oceanogr, 58(1):37-48, 2013.

[26] S. Haykin. Adaptive Filter Theory. Prentice Hall, 4th edition, 2001.

[27] J. Heidemann, M. Stojanovic, and M. Zorzi. Underwater sensor networks: applications, advances and challenges. Philosophical Transactions of the Royal Society A: Mathematical,Physical and Engineering Sciences, 370(1958):158-175, 2012. doi: 10.1098/rsta.2011.0214. URL http://rsta.royalsocietypublishing.org/ content/370/1958/158. abstract.

[28] J. B. Herbich and K. A. Ansari. Developments in Offshore Engineering: Wave Phenomena and Offshore Topics. Handbook of Coastal and Ocean Engineering Series. Gulf Publishing Company, 1999. ISBN 9780884153801. URL http://books.google. nl/books?id=fay-jVNpQfwC.

[29] F. Hlawatsch and G. Matz. Wireless communications over rapidly time-varying channels. Elsevier, 2011.

[30] R. Johnson, P. Schniter, T. J. Endres, J. D. Behm, D. R. Brown, and R. A. Casas. Blind equalization using the constant modulus criterion: A review. Proceedings of the IEEE, 86(10):1927-1950, 1998.

[31] D. L. Jones. A normalized constant-modulus algorithm. In Signals, Systems and Computers, 1995. 1995 Conference Record of the Twenty-Ninth Asilomar Conference on, volume 1, pages 694-697. IEEE, 1995.

[32] T. Kang, H. C. Song, and W. S. Hodgkiss. Long-range multi-carrier acoustic communication in deep water using a towed horizontal array. The Journal of the Acoustical Society of America, 131(6):4665-4671, 2012.

[33] R. A. Kennedy and Z. Ding. Blind adaptive equalizers for quadrature amplitude modulated communication systems based on convex cost functions. Optical Engineering, 31(6):1189-1199, 1992.

[34] W. C. Knight, R. G. Pridham, and S. M. Kay. Digital signal processing for sonar. Proceedings of the IEEE, 69(11):1451-1506, 1981.

[35] Koninklijk Nederlands Meteorologisch Instituut (Royal Netherlands Meteorological Institute). Jaaroverzicht van het weer in Nederland. Online, 2010. URL http: //www.knmi.nl/klimatologie/mow/pdf/jow_2010.pdf.

[36] M. J. Kruijswijk. Hierarchical wideband beamforming using fixed weights. Master's thesis, University of Twente, March 2011.

[37] W. A. Kuperman and J. F. Lynch. Shallow-water acoustics. Physics Today, 57(10):55-61, 2004. doi: 10.1063/1.1825269. URL http://link . aip.org/link/?PT0/57/55/1.

[38] S. Lambotharan and J. A. Chambers. A new blind equalization structure for deepnull communication channels. Circuits and Systems II: Analog and Digital Signal Processing, IEEE Transactions on, 45(1):108-114, 1998. 
[39] T. Leighton. The Acoustic Bubble. Academic Press, 1994. ISBN 9780124419209. URL http://books .google.nl/books?id=Z7eQy2LK8uMC.

[40] P. A. Lewin, M. E. Schafer, and R. C. Chivers. Factors affecting the choice of preamplification for ultrasonic hydrophone probes. Ultrasound in Medicine \& Biology, 13 (3):141 - 148, 1987. ISSN 0301-5629. doi: 10.1016/0301-5629(87)90142-6.

[41] Y. Li and Z. Ding. Convergence analysis of finite length blind adaptive equalizers. Signal Processing, IEEE Transactions on, 43(9):2120-2129, Sep 1995. ISSN 1053-587X. doi: $10.1109 / 78.414774$.

[42] R. Lindsay. Acoustics: Historical and Philosophical Development. Benchmark Papers in acoustics. Dowden, Hutchinson and Ross, 1972. URL http://books.google. nl/books? id=1xP9XwAACAAJ.

[43] W. Liu and S. Weiss. Wideband Beamforming. Wiley, 2010.

[44] R. Ljøen and A. Svansson. Long-term variations of subsurface temperatures in the skagerrak. Deep Sea Research and Oceanographic Abstracts, 19(4):277 - 288, 1972. ISSN o011-7471. doi: http://dx.doi.org/10.1016/oo11-7471(72)90021-6. URL http: //www.sciencedirect.com/science/article/pii/0011747172900216.

[45] R. A. Locarnini, A. V. Mishonov, J. I. Antonov, T. P. Boyer, H. E. Garcia, O. K. Baranova, M. M. Zweng, , and D. R. Johnson. World Ocean Atlas 2009, Volume 1: Temperature. U.S. Government Printing Office, 2010. Ed. NOAA Atlas NESDIS 68.

[46] K. V. Mackenzie. Nine-term equation for sound speed in the oceans. The Journal of the Acoustical Society of America, 70:807, 1981.

[47] H. Meikle. Modern Radar Systems. Artech House radar library. Artech House, 2001. ISBN 9781580532945. URL http://books.google.nl/books?id=M-040V2_ 9hEC.

[48] T. Milligan. Modern Antenna Design. Wiley, 2005. ISBN 9780471720607. URL http://books.google.nl/books?id=PPyDQXAd09kC.

[49] B. Molnar, I. Frigyes, Z. Bodnar, and Z. Herczku. The WSSUS channel model: comments and a generalisation. In Global Telecommunications Conference, 1996. GLOBECOM'96.'Communications: The Key to Global Prosperity, pages 158-162. IEEE, 1996.

[50] P. Morse and K. Ingard. Theoretical Acoustics. International series in pure and applied physics. Princeton University Press, 1986. ISBN 9780691024011. URL http: //books.google.nl/books?id=KIL4MV9IE5kC.

[51] D. Munoz, F. B. Lara, C. Vargas, and R. Enriquez-Caldera. Position Location Techniques and Applications. Elsevier Science, 2009. ISBN 9780080921938. URL http://books . google.nl/books?id=2sWdiZainyoC.

[52] A. K. Nandi. Blind Estimation using Higher-Order Statistics. Kluwer Academic Publishers, 2010.

[53] Nederlandse Organisatie voor Toegepast Natuurwetenschappelijk Onderzoek (Netherlands Organisation for Applied Scientific Research). Pile driving monitoring. (on request). 
[54] L. K. Nguyen and R. B. Wells. Doppler shift cancellation using phasor and split phasor LMS algorithms. In Military Communications Conference, 2008. MILCOM 2008. IEEE, pages 1-6, Nov. 2008. doi: 10.1109/MILCOM.2008.4753242.

[55] C. Nilsen and I. Hafizovic. Beamspace adaptive beamforming for ultrasound imaging. Ultrasonics, Ferroelectrics and Frequency Control, IEEE Transactions on, 56(10):21872197, 2009. ISSN 0885-3010. doi: 10.1109/TUFFC.2009.1301.

[56] J. Northrop and J. Colborn. Sofar channel axial sound speed and depth in the Atlantic Ocean. Journal of Geophysical Research, 79(36):5633-5641, 1974.

[57] O. of Scientific Research and D. N. D. R. Committee. Summary Technical Report of Division 6, NDRC. Washington, D.C., United States., 1946. URL http://books . google.nl/books?id=LjeMuAAACAAJ.

[58] N. Parrish, S. Roy, W. L. J. Fox, and P. Arabshahi. Rate-range for an FH-FSK Acoustic Modem. In Proceedings of the Second Workshop on Underwater Networks, WuWNet 'o7, pages 93-96, New York, NY, USA, 2007. ACM. ISBN 978-1-59593-736o. doi: 10.1145/1287812.1287832. URL http://doi.acm.org/10.1145/1287812. 1287832 .

[59] A. Paulraj, R. Roy, and T. Kailath. A subspace rotation approach to signal parameter estimation. Proceedings of the IEEE, 74(7):1044-1046, 1986. ISSN 0018-9219. doi: 10.1109/PROC.1986.13583.

[6o] A. D. Pierce. Extension of the method of normal modes to sound propagation in an almost-stratified medium. The Journal of the Acoustical Society of America, 37(1): 19-27, 1965. doi: 10.1121/1.1909303. URL http://link . aip.org/link/?JAS/37/ $19 / 1$.

[61] D. Pompili, T. Melodia, and I. F. Akyildiz. Deployment analysis in underwater acoustic wireless sensor networks. In WUWNet 'o6: Proceedings of the 1st ACM international workshop on Underwater networks, pages 48-55, New York, NY, USA, 2006. ACM. ISBN 1-59593-484-7. doi: http://doi.acm.org/10.1145/1161039.1161050.

[62] M. B. Porter. Bellhop gaussian beam/finite element beam code, 2007. URL http: //oalib.hlsresearch.com/rays.

[63] M. B. Porter and H. P. Bucker. Gaussian beam tracing for computing ocean acoustic fields. The Journal of the Acoustical Society of America, 82:1349, 1987.

[64] J. G. Proakis and D. K. Manolakis. Digital Signal Processing: Principles, Algorithms, and Applications. Prentice Hall, 1996.

[65] S. Renals, H. Bourlard, and J. Carletta. Multimodal Signal Processing: Human Interactions in Meetings. Multimodal Signal Processing: Human Interactions in Meetings. Cambridge University Press, 2012. ISBN 9781107022294. URL http: //books.google.nl/books?id=U015mW6C26oC.

[66] W. J. Richardson and D. H. Thomson. Marine Mammals and Noise. Academic Press, 1995. ISBN 9780125884419. URL http://books.google.nl/books?id= OIWmaG906jgC. 
[67] M. Rubsamen and A. B. Gershman. Direction-of-arrival estimation for nonuniform sensor arrays: From manifold separation to fourier domain music methods. Signal Processing, IEEE Transactions on, 57(2):588-599, 2009. ISSN 1053-587X. doi: 10.1109/TSP.2008.2008560.

[68] A. Rudge. The Handbook of Antenna Design: Volume 1. Institution of Electrical Engineers, 1982.

[69] Y. Sato. A method of self-recovering equalization for multilevel amplitudemodulation systems. Communications, IEEE Transactions on, 23(6):679-682, Jun 1975. ISSN oogo-6778. doi: 10.1109/TCOM.1975.1092854.

[7o] R. Schmidt. Multiple emitter location and signal parameter estimation. Antennas and Propagation, IEEE Transactions on, 34(3):276-280, Mar 1986. ISSN oo18-926X.

[71] O. Shalvi and E. Weinstein. New criteria for blind deconvolution of nonminimum phase systems (channels). Information Theory, IEEE Transactions on, 36(2):312-321, 1990.

[72] C. H. Sherman and J. L. Butler. Transducers and Arrays for Underwater Sound. Monograph Series in Underwater Acoustics. Springer Science+Business Media, LLC, 2007. ISBN 9780387331393. URL http://books.google.nl/books?id= SrREi-ScbFcC.

[73] B. Sklar. Rayleigh fading channels in mobile digital communication systems part ii: Mitigation. Communications Magazine, IEEE, 35(9):148 -155, sept. 1997. ISSN 0163-6804. doi: 10.1109/MCOM.1997.621038.

[74] M. Skolnik. Radar Handbook, Third Edition. Electronics electrical engineering. McGraw-Hill Education, 2008. ISBN 9780071485470. URL http: //books . google. $\mathrm{nl} /$ books?id=76uF2Xebm-gC.

[75] B. Smolders and G. Hampson. Deterministic rf nulling in phased arrays for the next generation of radio telescopes. Antennas and Propagation Magazine, IEEE, 44(4): 13-22, Aug 2002. ISSN 1045-9243. doi: 10.1109/MAP.2002.1043143.

[76] E. M. Sözer and M. Stojanovic. Reconfigurable Acoustic Modem for Underwater Sensor Networks. In Proceedings of the 1st ACM international workshop on Underwater networks, WUWNet '06, pages 101-104, New York, NY, USA, 2006. ACM. ISBN 1-59593-484-7. doi: http://doi.acm.org/10.1145/1161039.116106o.

[77] M. Stojanovic. Underwater acoustic communications. In Electro/95 International. Professional Program Proceedings., pages 435-440, 1995. doi: 10.1109/ELECTR.1995.471021.

[78] M. Stojanovic. On the relationship between capacity and distance in an underwater acoustic communication channel. SIGMOBILE Mob. Comput. Commun. Rev., 11:3443, October 2007. ISSN 1559-1662. doi: http://doi.acm.org/10.1145/1347364.1347373.

[79] M. Stojanovic and J. Preisig. Underwater acoustic communication channels: Propagation models and statistical characterization. Communications Magazine, IEEE, 47 (1):84 -89, 2009. ISSN 0163-6804. doi: 10.1109/MCOM.2009.4752682. 
[8o] R. Stolkin, A. Sutin, S. Radhakrishnan, M. Bruno, B. Fullerton, A. Ekimov, and M. Raftery. Feature based passive acoustic detection of underwater threats. pages 620408-620408-10, 2006. doi: 10.1117/12.663651. URL +http://dx. doi.org/10. $1117 / 12.663651$.

[81] S. Talwar, M. Viberg, and A. Paulraj. Blind estimation of multiple co-channel digital signals arriving at an antenna array. In Signals, Systems and Computers, 1993. 1993 Conference Record of The Twenty-Seventh Asilomar Conference on, pages 349-353 vol.1, Nov 1993. doi: 10.1109/ACSSC.1993.342532.

[82] Terasic. DE0-Nano Development and Education Board. Online, December 2013. URL www. terasic.com.tw/en/.

[83] S. Theodoridis and R. Chellappa. Academic Press Library in Signal Processing: Volume 3: Array and Statistical Signal Processing. Academic Press Library in Signal Processing. Elsevier Science, 2013. ISBN 9780124116214. URL http://books.google.nl/ books?id=FpEHw9aemekC.

[84] J. Treichler and B. Agee. A new approach to multipath correction of constant modulus signals. Acoustics, Speech and Signal Processing, IEEE Transactions on, 31(2):459-472, Apr 1983. ISSN 0096-3518.

[85] J. F. Tressler. Piezoelectric transducer designs for sonar applications. In Piezoelectric and Acoustic Materials for Transducer Applications, pages 217-239. Springer US, 2008. ISBN 978-0-387-76538-9. doi: 10.1007/978-0-387-76540-2_11. URL http://dx . doi . org/10.1007/978-0-387-76540-2_11.

[86] R. Urick. Principles Of Underwater Sound. McGraw-Hill Ryerson, Limited, 1983. ISBN 9780070660878. URL http://books.google.nl/books?id= hfXQAAAAMAAJ.

[87] A.-J. van der Veen. Signal Processing for Communications. Delft University of Technology, 2004. Reader ET4 147.

[88] A.-J. van der Veen and A. Paulraj. An analytical constant modulus algorithm. Signal Processing, IEEE Transactions on, 44(5):1136-1155, 1996.

[89] H. L. van Trees. Optimum Array Processing. Wiley, 2002.

[9o] B. D. Van Veen and K. M. Buckley. Beamforming: A versatile approach to spatial filtering. ASSP Magazine, IEEE, 5(2):4-24, 1988.

[91] D. B. Ward and T. Abhayapala. Range and bearing estimation of wideband sources using an orthogonal beamspace processing structure. In Acoustics, Speech, and Signal Processing, 2004. Proceedings. (ICASSP '04). IEEE International Conference on, volume 2, pages ii-109-12 vol.2, 2004. doi: 10.1109/ICASSP.2004.1326206.

[92] J. L. Worzel, C. L. Pekeris, and W. M. Ewing. Propagation of Sound in the Ocean: Explosion Sounds in Shallow Water. Number v. 27 in Contribution ... from the Woods Hole Oceanographic Institution. Geological Society of America, 1948. URL http: //books. google.nl/books?id=BPrvAAAAMAAJ.

[93] Y. Xiao, editor. Underwater Acoustic Sensor Networks. Auerbach Publications, 2010. 
[94] Z. Xu. New cost function for blind estimation of M-PSK signals. In Wireless Communications and Networking Conference, 200o. WCNC. 200o IEEE, volume 3, pages 1501-1505 vol.3, 2000. doi: 10.1109/WCNC.2000.904857.

[95] H. Yang, Z. Lin, and X. Cai. Design of a QPSK demodulator for DVB-S receiver ASIC chip. In Solid-State and Integrated Circuits Technology, 2004. Proceedings. 7th International Conference on, volume 3, pages 2075-2078 vol.3, Oct. 2004. doi: 10.1109/ICSICT.2004.1435252.

[96] J.-T. Yuan and T.-C. Lin. Equalization and carrier phase recovery of cma and mma in blind adaptive receivers. Signal Processing, IEEE Transactions on, 58(6):3206-3217, 2010.

[97] V. Zarzoso and P. Comon. Blind channel equalization with algebraic optimal step size. In in Proc. XIII Eur. Signal Process. Conf, 2005.

[98] I. Ziskind and M. Wax. Maximum likelihood localization of multiple sources by alternating projection. Acoustics, Speech and Signal Processing, IEEE Transactions on, 36(10):1553-156o, Oct. 1988. ISSN oo96-3518. doi: 10.1109/29.7543. 


\section{List of Publications}

[KCH:1] K. C. H. Blom, M. D. van de Burgwal, K. C. Rovers, A. B. J. Kokkeler, and G. J. M. Smit. DVB-S Signal Tracking Techniques for Mobile Phased Arrays. In IEEE 72nd Vehicular Technology Conference Fall (VTC 2010-Fall), Ottawa, ON, Canada, page 5, USA, September 2010. IEEE Vehicular Technology Society.

[KCH:2] K. C. H. Blom, M. D. van de Burgwal, K. C. Rovers, A. B. J. Kokkeler, and G. J. M. Smit. Angular CMA: A modified Constant Modulus Algorithm providing steering angle updates. In Seventh International Conference on Wireless and Mobile Communications, ICWMC 2011, Luxembourg, pages 42-47. Xpert Publishing Services, June 2011.

[KCH:3] K. C. H. Blom, M. E. T. Gerards, A. B. J. Kokkeler, and G. J. M. Smit. Nonminimum-phase channel equalization using All-Pass CMA. In 2013 IEEE 24th International Symposium on Personal, Indoor and Mobile Radio Communications: Fundamentals and PHY Track (PIMRC'13 - Fundamentals and PHY Track), London, United Kingdom, 2013.

[KCH:4] K. C. H. Blom, R. Wester, A. B. J. Kokkeler, and G. J. M. Smit. Low-cost multichannel underwater acoustic signal processing testbed. In 7 th IEEE Sensor Array and Multichannel Signal Processing Workshop (SAM), 2012, Hoboken, NJ, USA, pages 505-508, USA, July 2012. IEEE.

[KCH:5] W. A. P. van Kleunen, K. C. H. Blom, N. Meratnia, A. B. J. Kokkeler, P. J. M. Havinga, and G. J. M. Smit. Underwater Localization by combining time-of-flight and direction-of-arrival. In OCEANS 2014, MTS/IEEE Taipei, 2014. (submitted for review).

[KCH:6] M. D. van de Burgwal, K. C. Rovers, K. C. H. Blom, A. B. J. Kokkeler, and G. J. M. Smit. Adaptive Beamforming using the Reconfigurable Montium TP. In S. López, editor, Proceedings of the 13th Euromicro Conference on Digital System Design, Lille, France, pages 301-308. IEEE Computer Society, September 2010.

[KCH:7] M. D. van de Burgwal, K. C. Rovers, K. C. H. Blom, A. B. J. Kokkeler, and G. J. M. Smit. Mobile Satellite Reception with a Virtual Satellite Dish based on a Reconfigurable Multi-Processor Architecture. Microprocessors and Microsystems, 35(8): 716-728, September 2011. ISSN 0141-9331. 




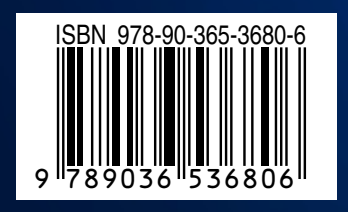




\section{STELLINGEN}

behorende bij het proefschrift

\section{Blind Equalization for Underwater Communications door Koen C.H. Blom, te verdedigen op vrijdag 4 juli 2014}

1 - Blinde adaptieve equalisatie is een veelbelovende aanpak om energie-efficiënt te compenseren voor de verstoring die veroorzaakt wordt door het akoestische onderwaterkanaal.

(Hoofdstuk 4)

2 - Parameterisatie van blinde adaptieve equalisatiemethoden is een effectieve strategie om zowel de dimensionaliteit als de gemiddelde convergentietijd van deze methoden te beperken.

(Hoofdstuk 5 en 6)

3 - Hiërarchische mixed-signal bundelvorming met digitale adaptieve gewichtsbepaling biedt een interessante architectuur voor kosten- en energie-efficiënte spatiële equalisatie.

4 - Het realiseren van transparante communicatie tussen wetenschappelijke onderzoeksgroepen, die veelal hun eigen belangen vooropstellen, is zeker niet eenvoudiger dan onderwatercommunicatie.

5 - Puur het implementeren van bestaande wetenschappelijke kennis kan promovendi sturing geven, maar mag nooit het hoofddoel worden.

6 - De hoeveelheid wetenschappelijke discussie in de pauze is omgekeerd evenredig aan de diversiteit aan onderzoeksthema's in de desbetreffende onderzoeksgroep.

7 - Digital signal processing is a clear-cut example of the importance of abstract mathematics in modern life and communications.

(Anonymous)

8 - The real voyage of discovery consists not in seeking new landscapes, but in having new eyes.

(Marcel Proust)

9 - Het zijn vaak de personen die nooit met het openbaar vervoer reizen, die er het meest over klagen. 